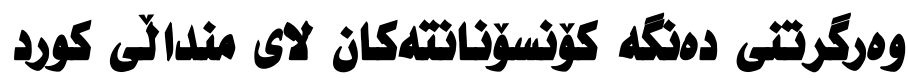

كارزاز خدر اسماعيل

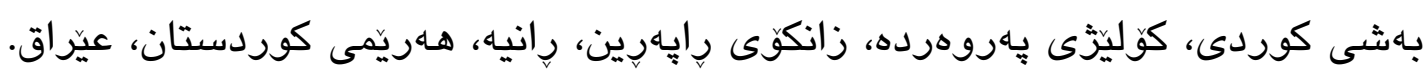
ئيمهيل: karzankhdir2@gmail.com

شيروان حسيّز حمد

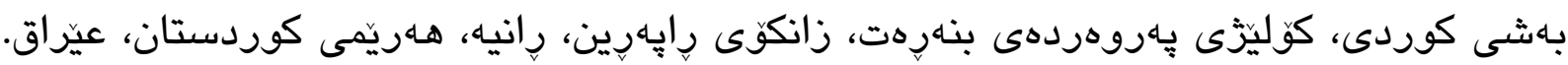
ئيمهيل: sherwan983@uor.edu.krd

هوخته:

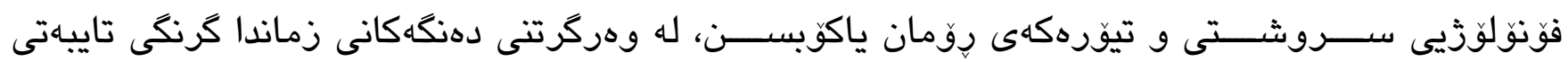

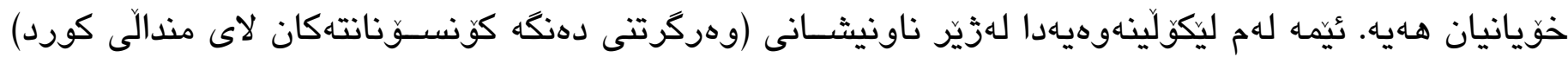

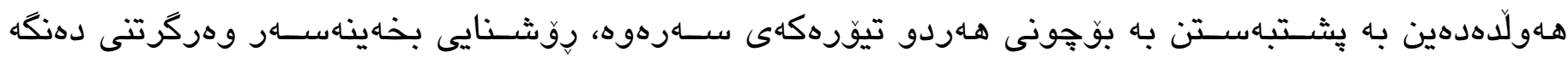

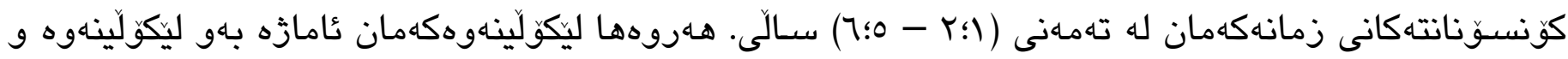

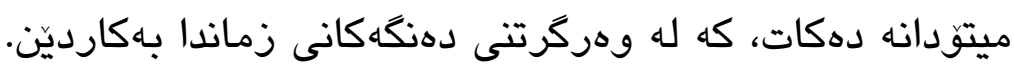

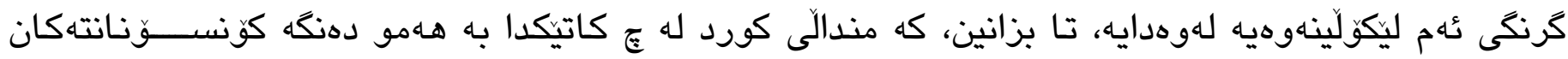

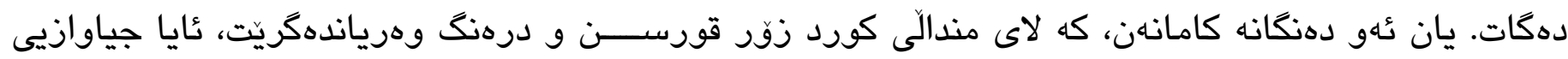

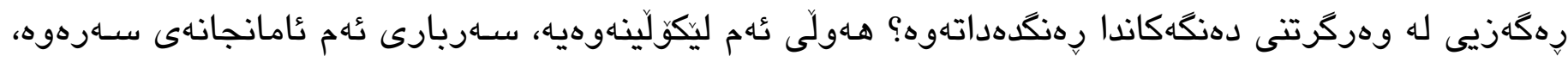

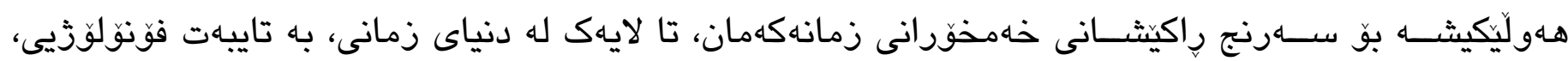

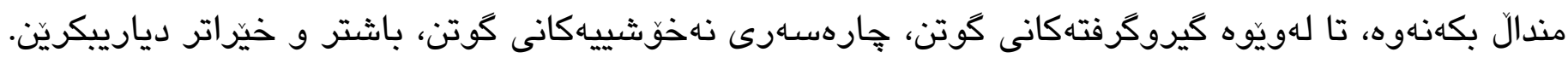

كليله وشُلهكان: فؤنولوّذيى سروشتى، دهنكه نيشانهكراو و نيشانهنهراوهكان، وهركرتنى كونسوّنانتهان. 


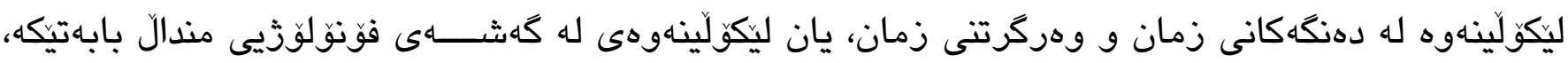

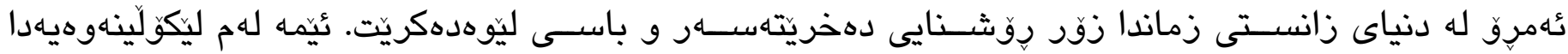

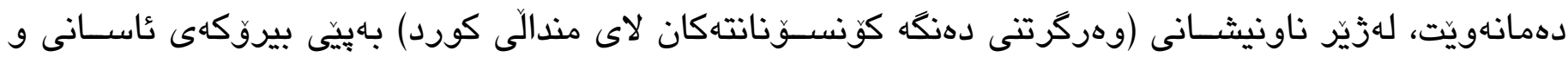

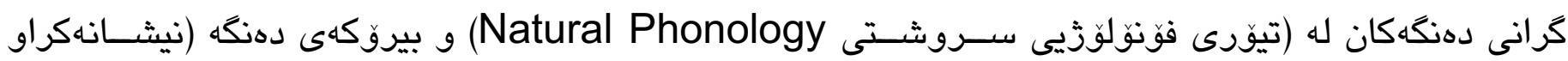

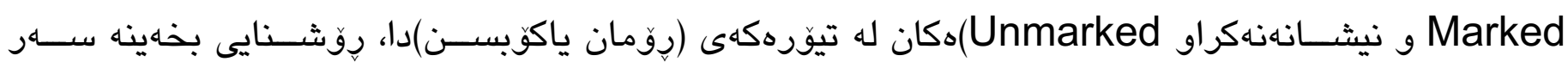

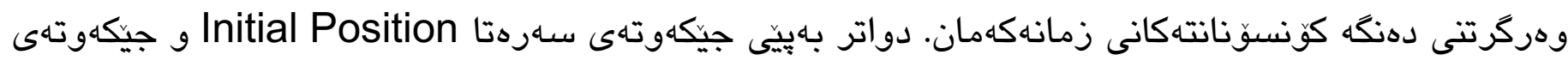

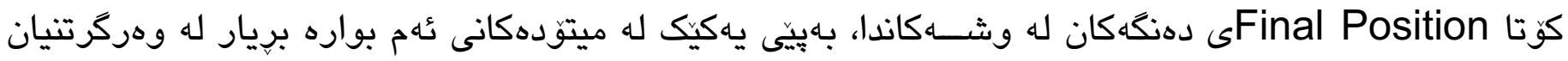

دهدهين.

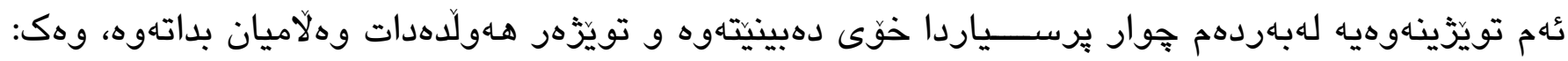

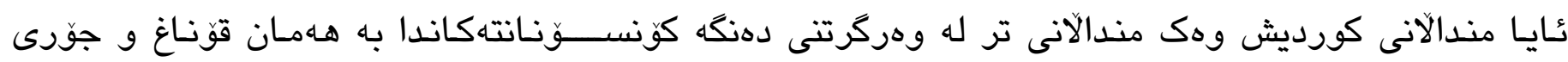

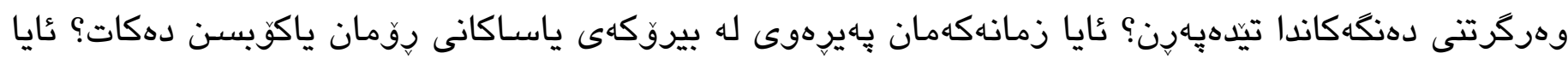

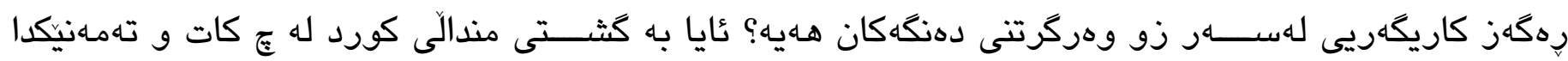

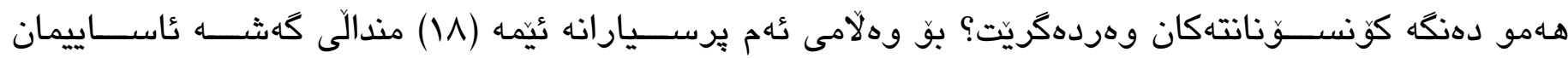

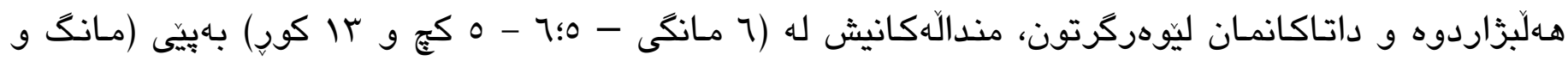

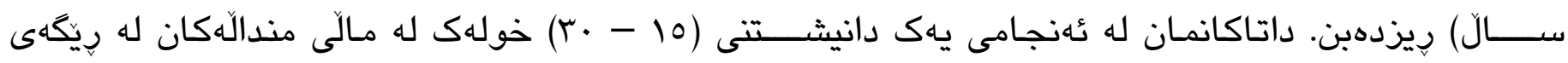

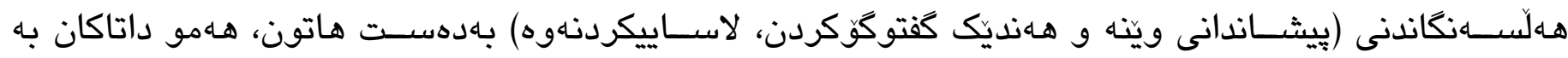

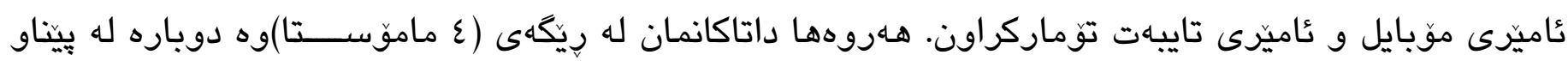

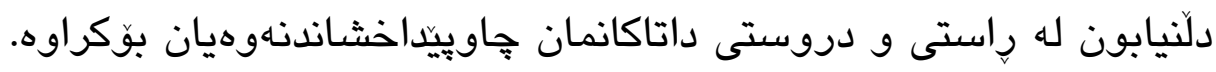

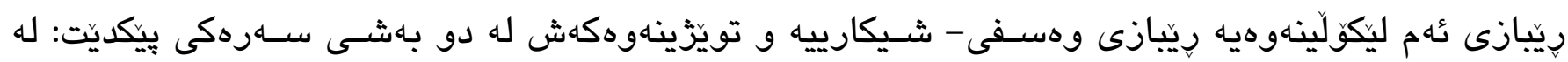

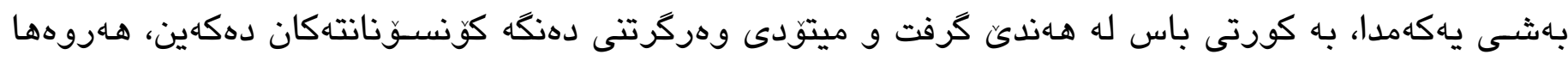

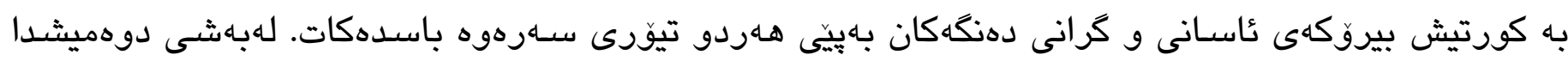

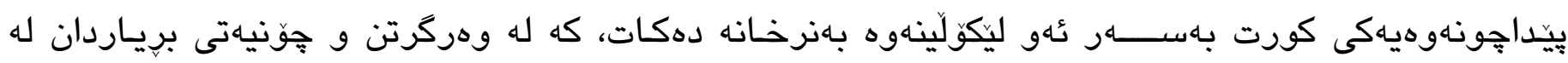

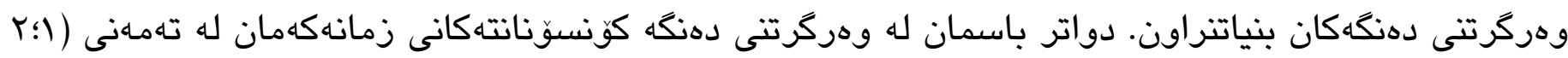

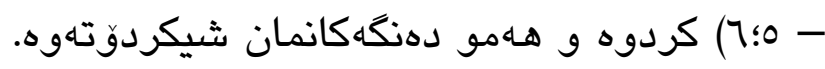

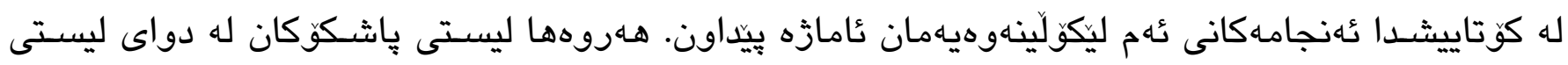

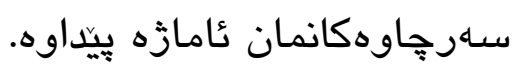

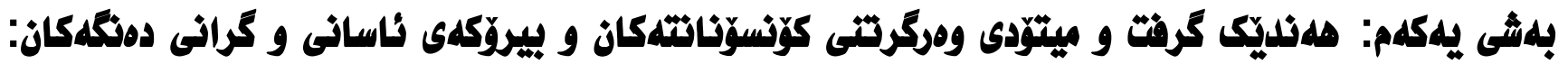

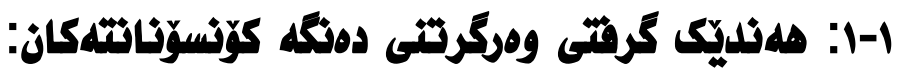

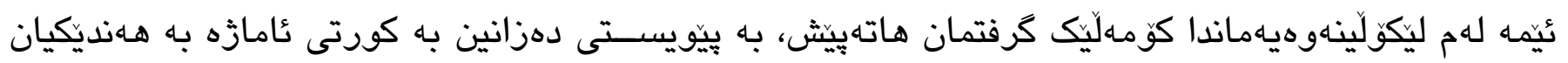
بكهين:

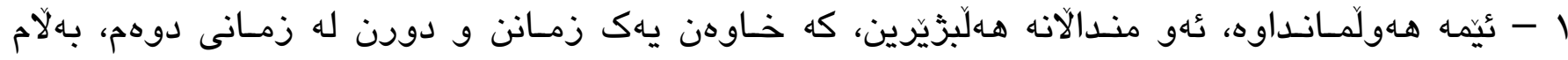

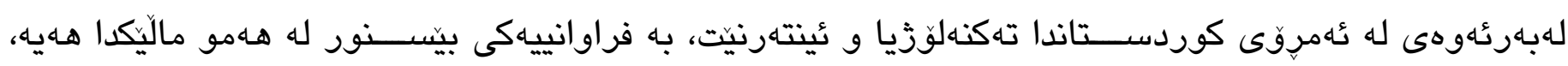

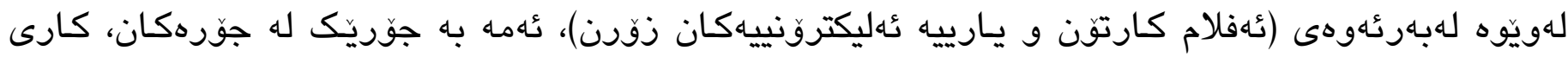




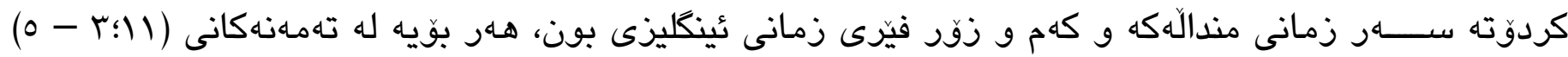

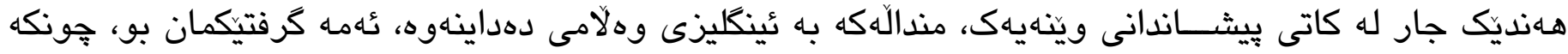

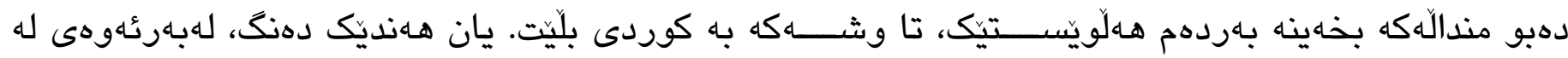

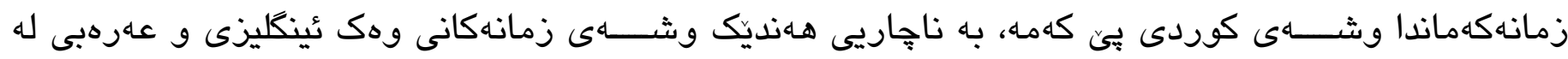
ويناهكاندا باهكار هيناوه.

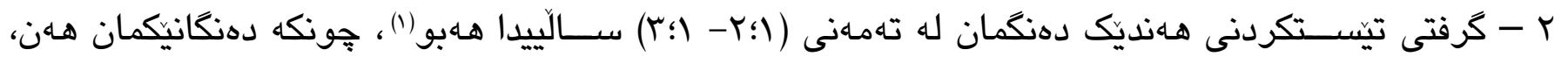

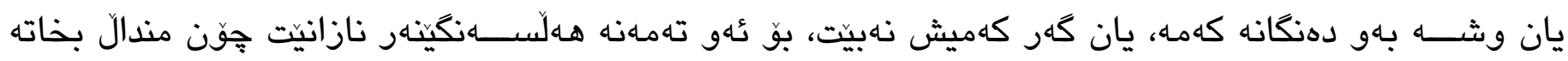

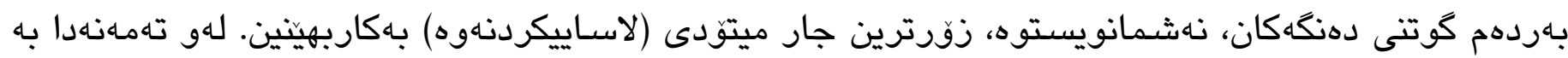

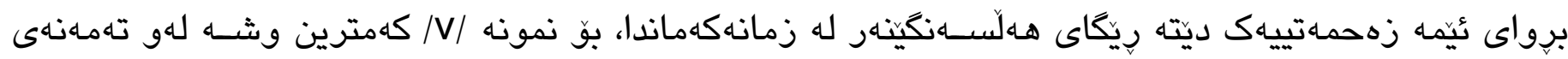

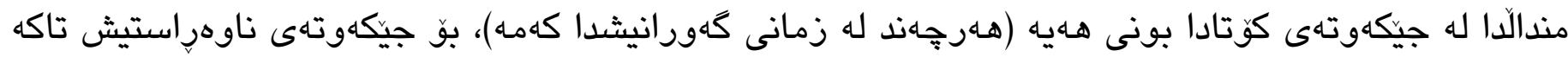

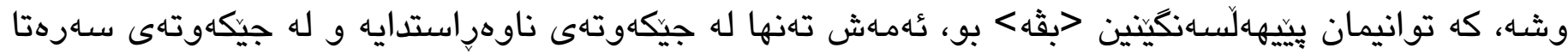

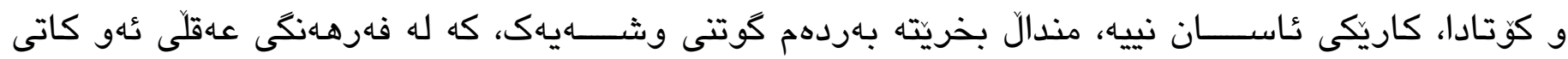

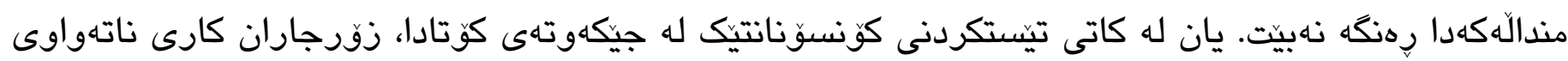

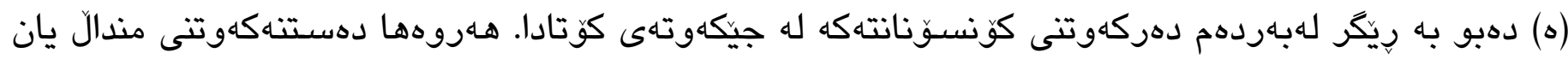

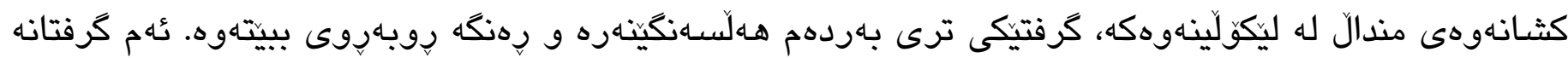

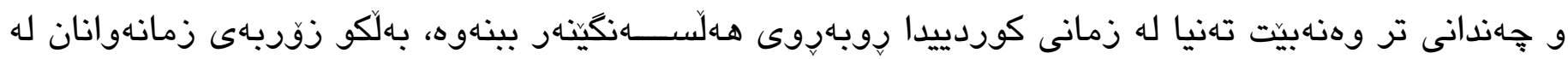

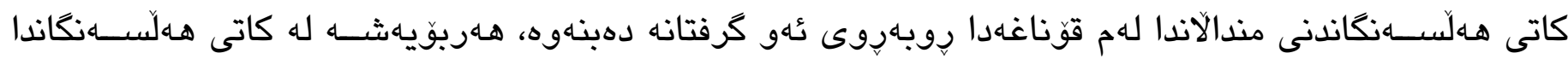

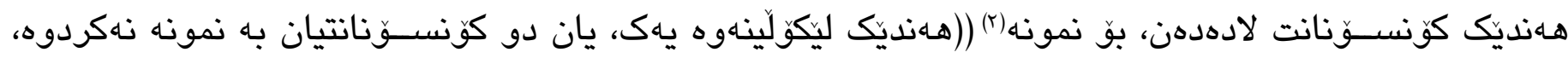
Smit et al 1990) (Bankson and Bernthal, 1990, 2019; Fudala, 2000; Fudala and Stegall, 2017

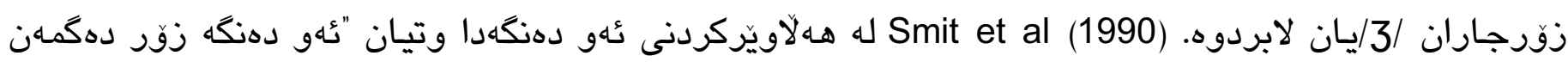

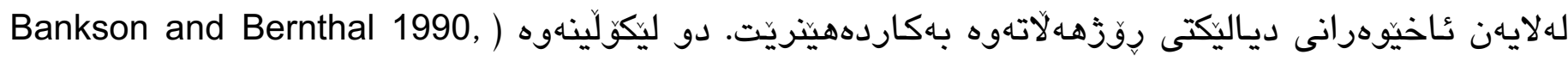

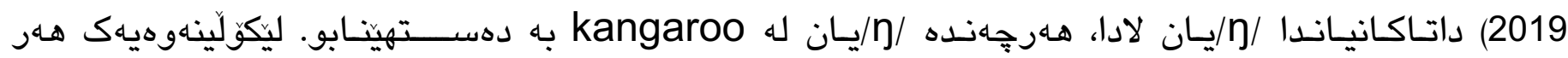

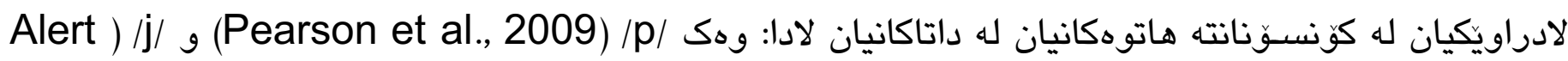

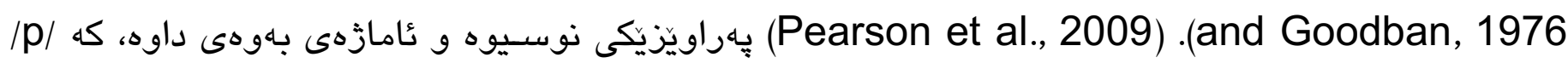

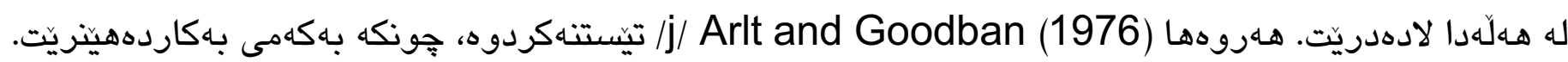

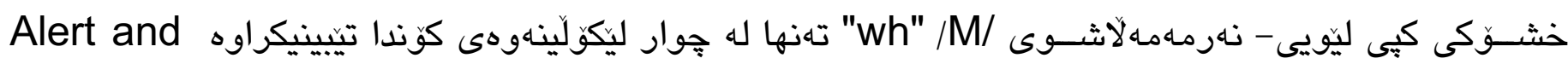
(Kathryn ((Wellman et al., 1931) (Goodban, 1976; Prather et al., 1975; Templin, 1976 ; Crowe, Sharynne Mcleod, 2020, 2160)

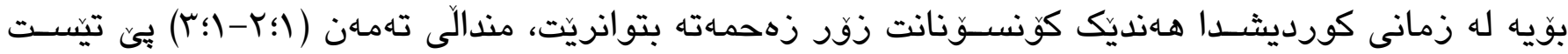

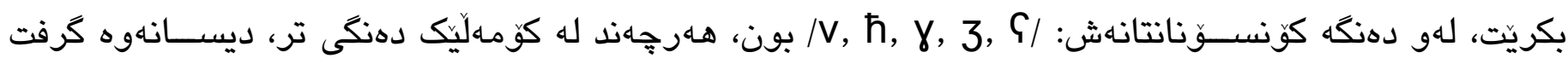

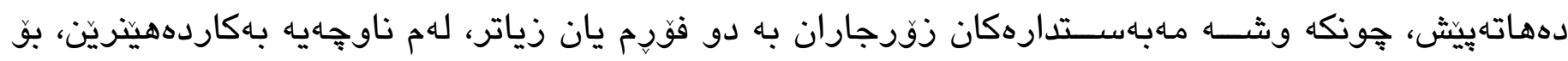

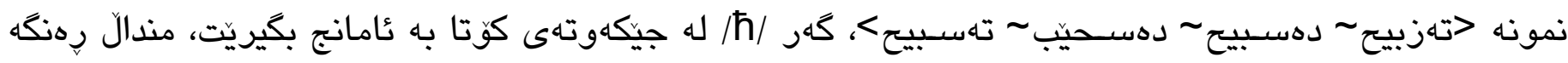

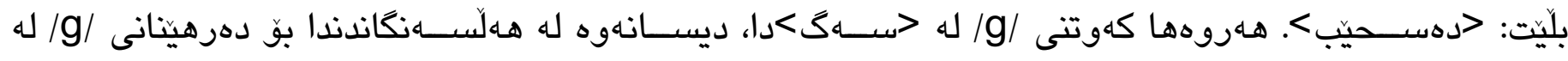

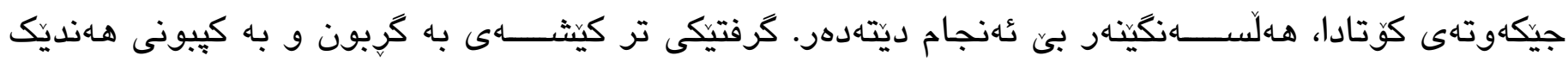

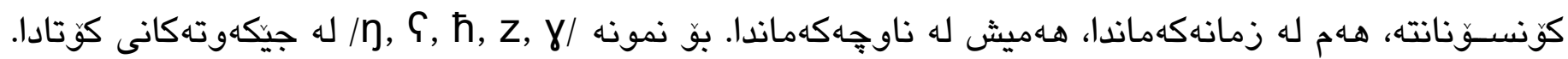




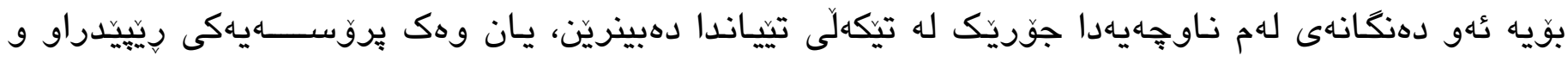

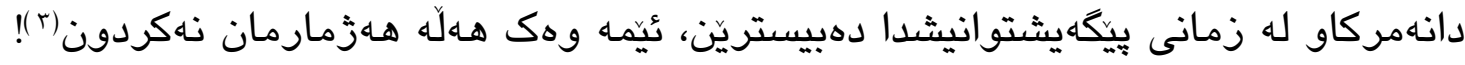

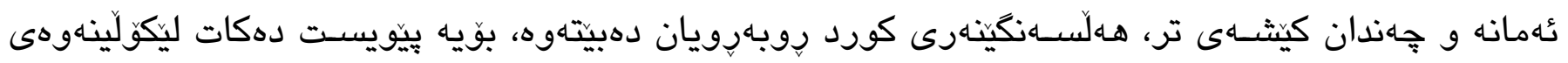
زياتر و دريّزتر لهم بوارهانه

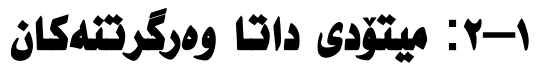

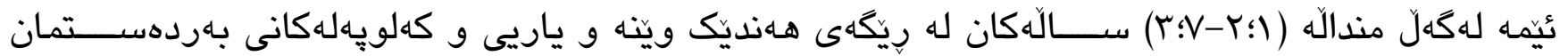

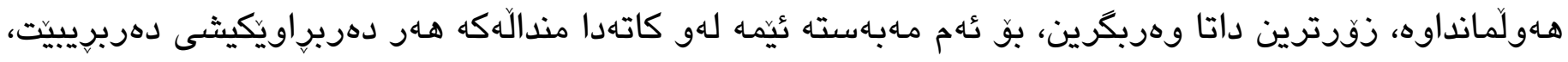

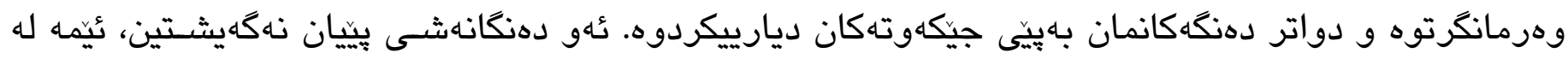

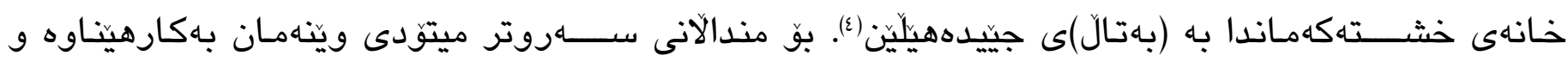

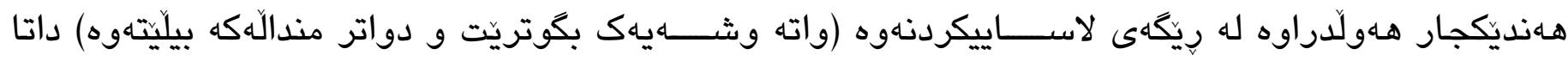

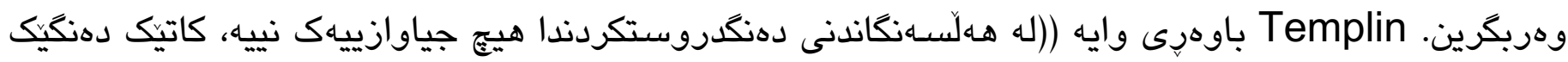

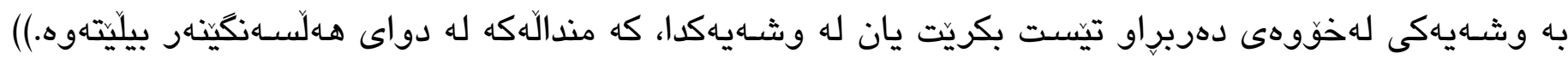

(Templin, 1947, 300)

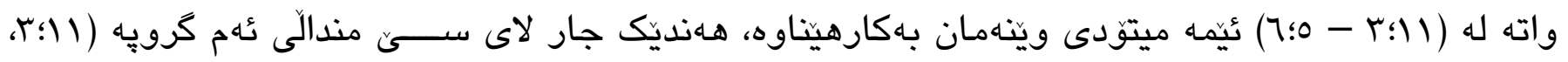

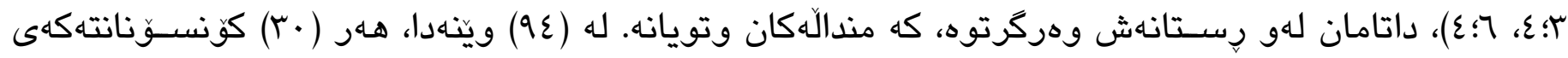

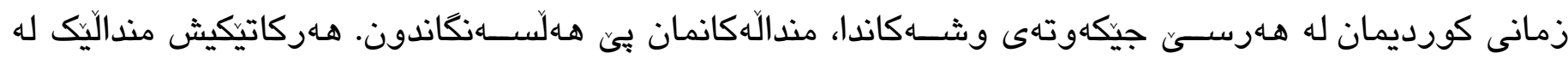

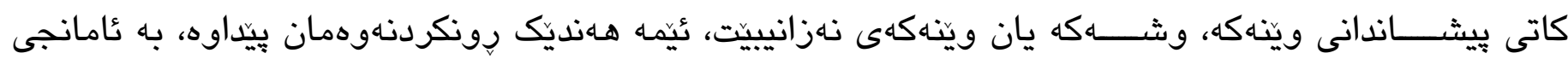

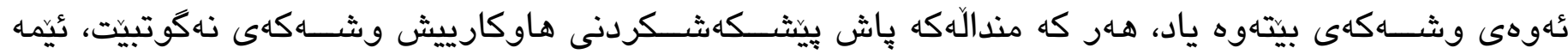

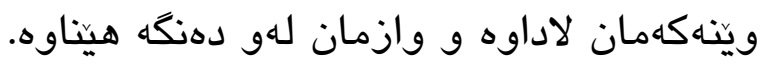

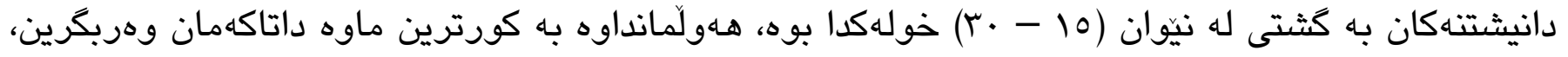

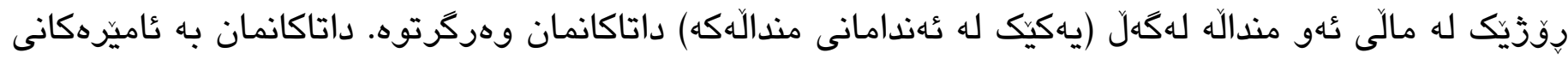

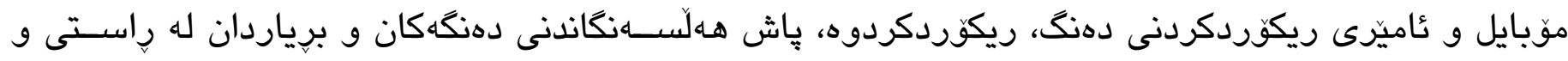

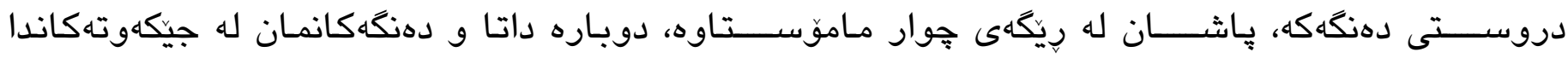

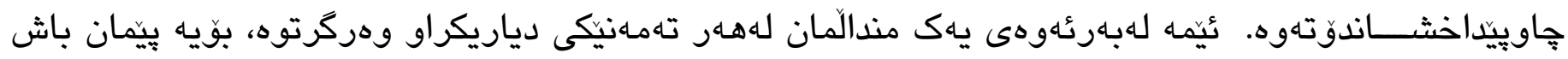

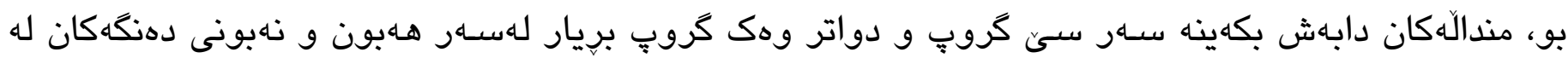

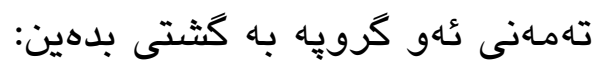

\begin{tabular}{|c|c|c|c|c|}
\hline$* S D$ & 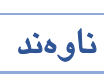 & بهشداربو & تهمهن/ مانك & كرويهكان \\
\hline V & זr & (ع كور و اكج) & $\varepsilon r-r_{0}$ & كرويى يهكهم \\
\hline r.A & or & (ع كور و اكج) & $\Delta V-\varepsilon V$ & كرويى دوهم \\
\hline $7 . r$ & $7 \Lambda$ & (r كَ و r كور) & $V V-71$ & كرويى سييهم \\
\hline
\end{tabular}

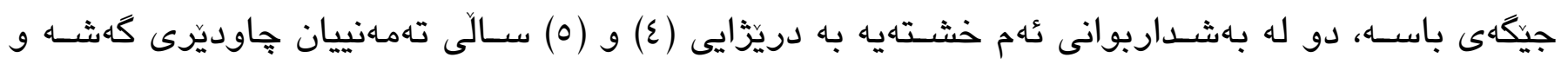

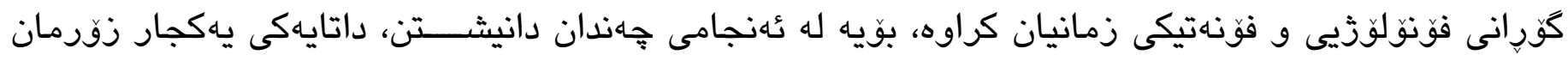

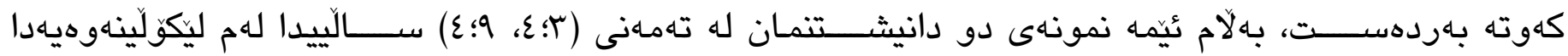




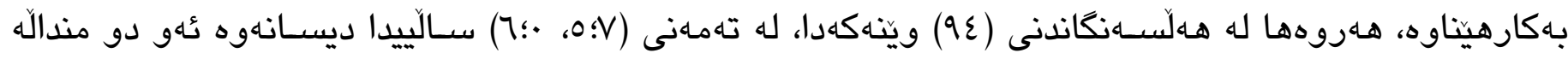

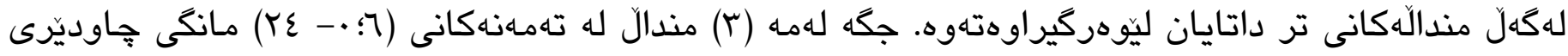

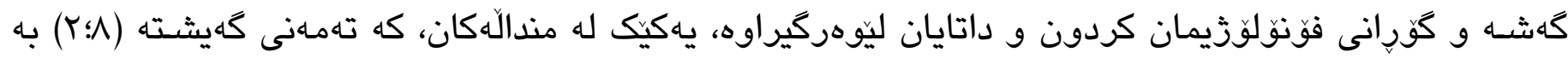
جيا داتاى ليّوهركيراوهتهوه.

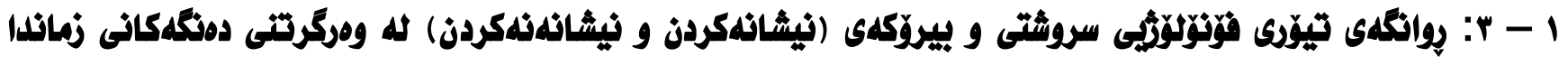

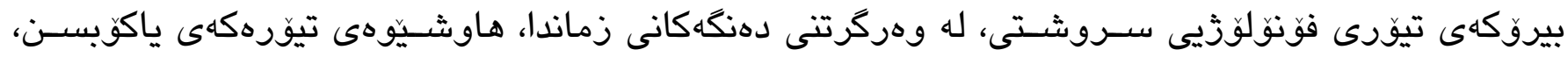

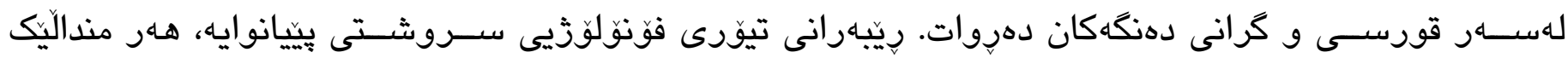

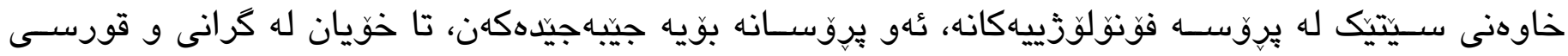

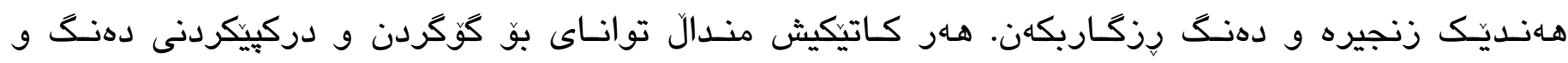

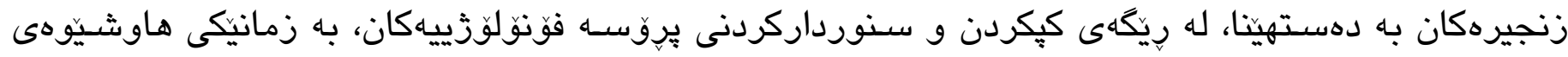

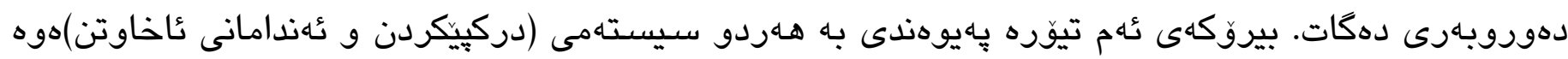

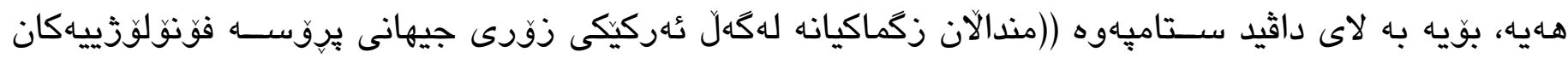

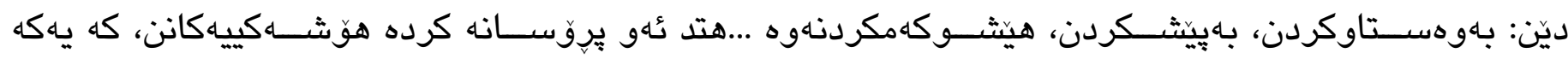

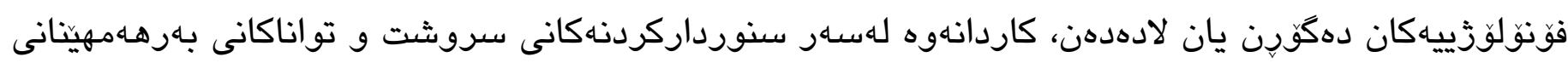

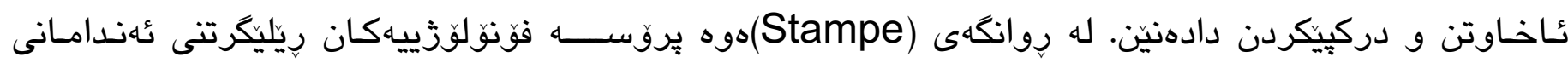

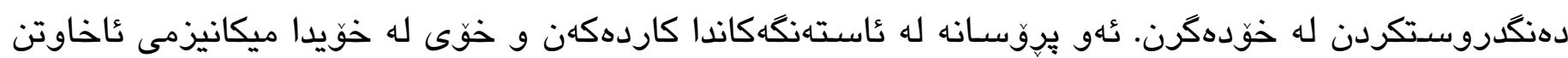

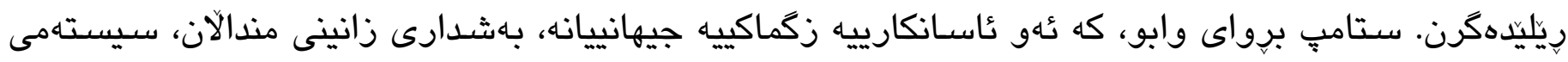

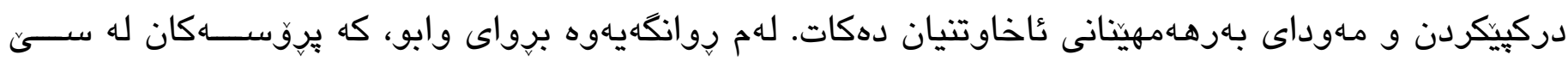

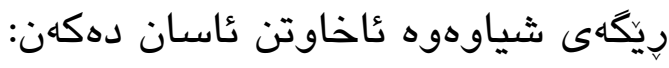

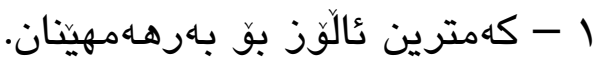
ب - كهمترين عالِّز بوّ دركينيكردن.

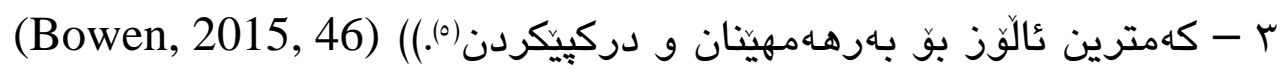

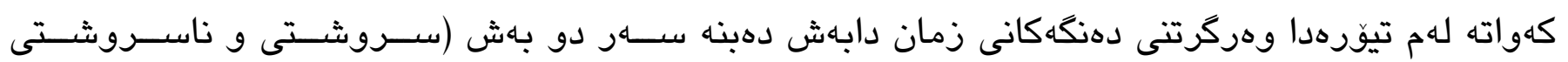
Natural and Unnatural

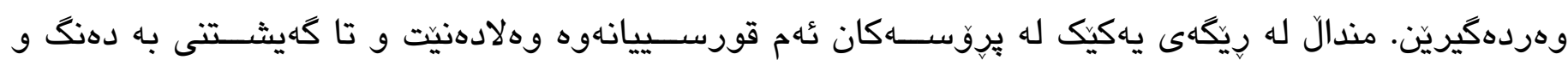

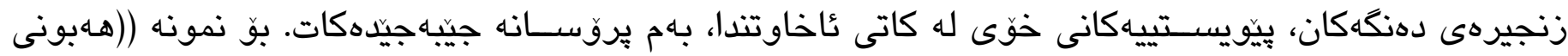

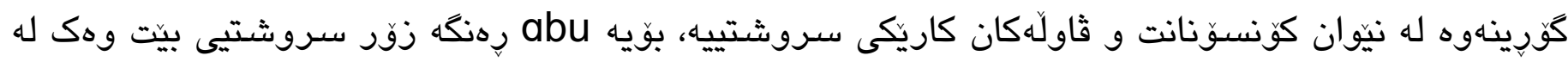

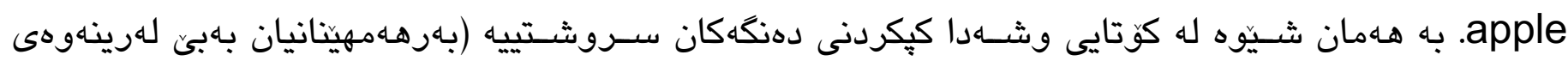

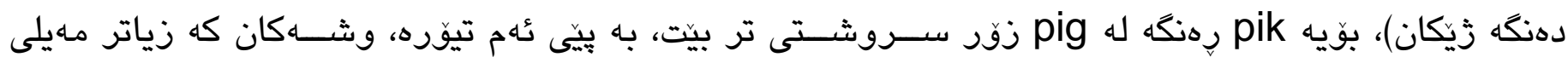

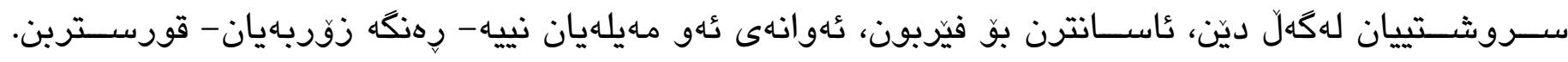

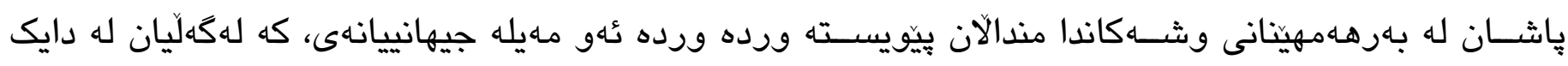

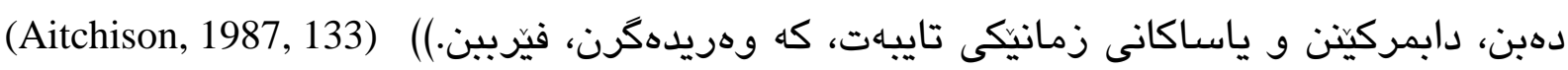

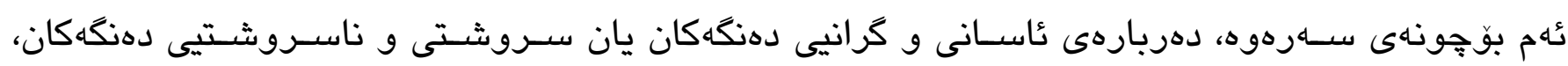

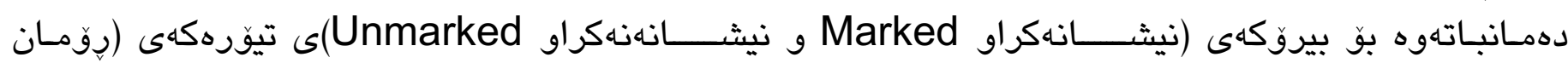




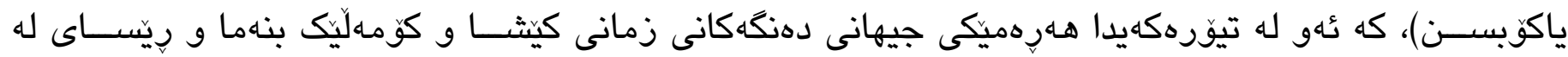

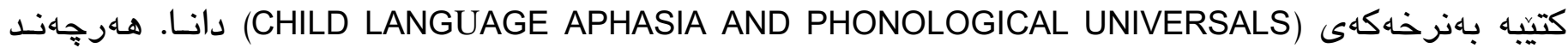

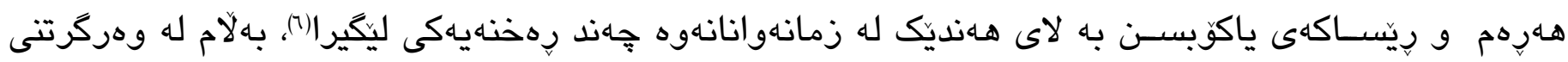

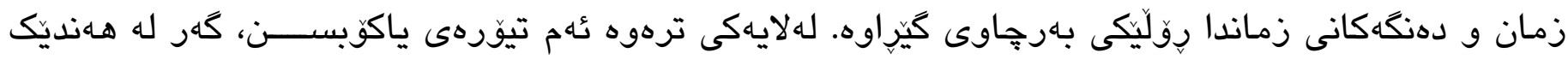

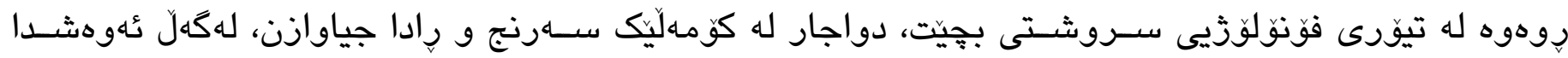

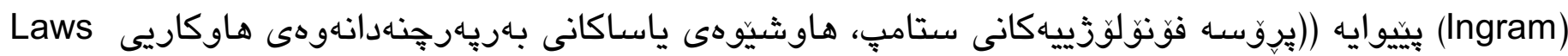
of irreversible solidarity

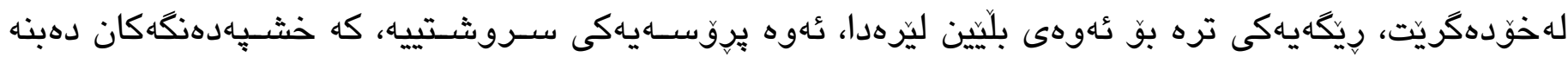
وهستاوهكان. Stampe ئهم

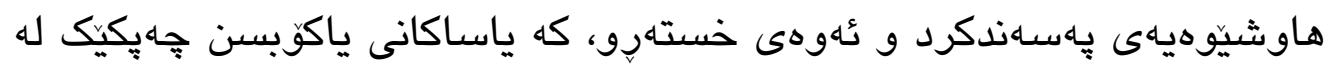

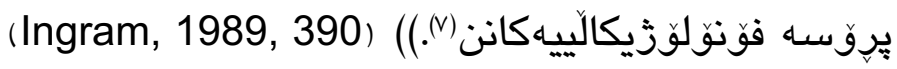

\begin{tabular}{|c|c|}
\hline 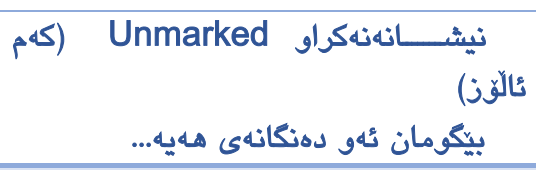 & 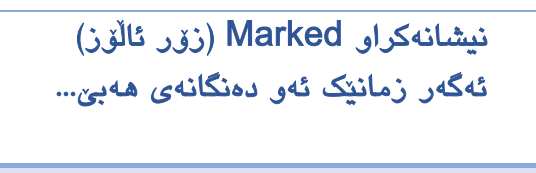 \\
\hline وهستاوهكان & خشّوكهكان \\
\hline خشوكه كان & ئهفريكات \\
\hline 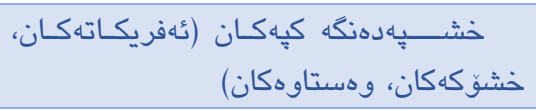 & 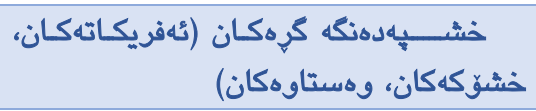 \\
\hline لوتييهكان & ليكويدهكان \\
\hline ئه فريكاتهكان & هيثبوه راستهقينهكان \\
\hline 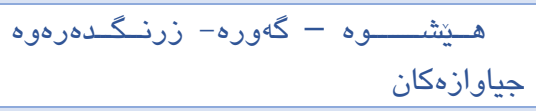 & 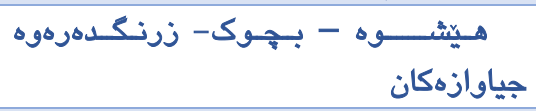 \\
\hline هيشتوه هاوسيّيةكان & هيشبوه راستهقينهان \\
\hline هيَشوه دو توخمهكان & هيشيوه سيى توخمهكان \\
\hline
\end{tabular}

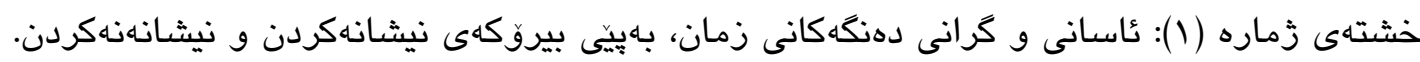

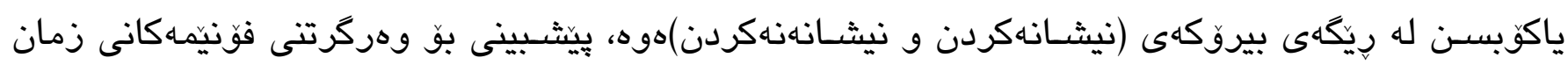

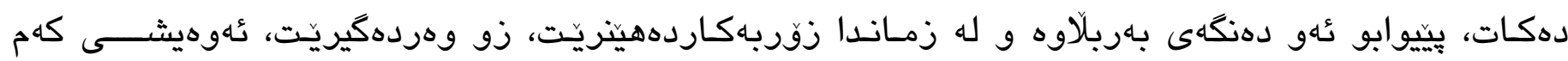

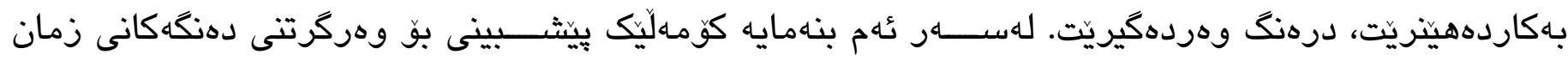

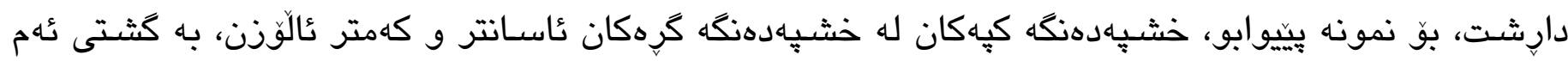

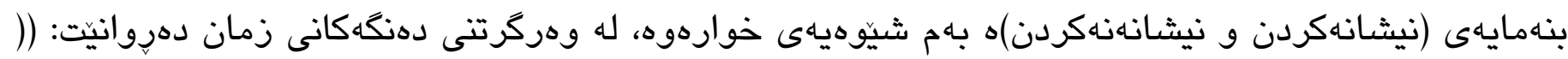

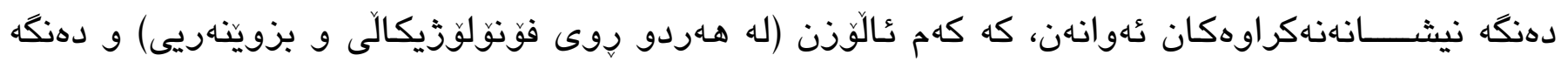

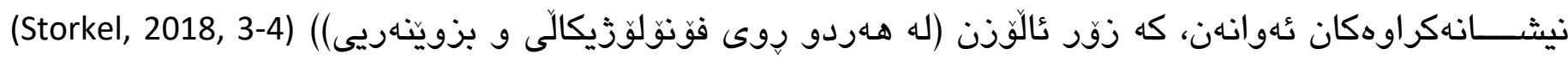

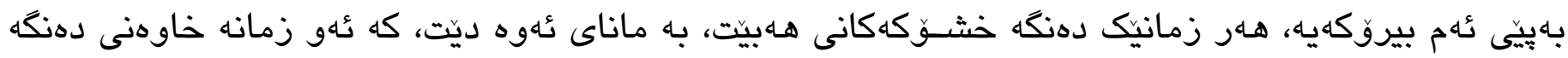




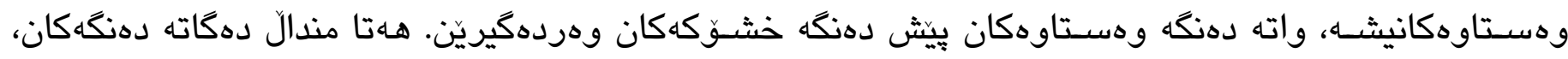

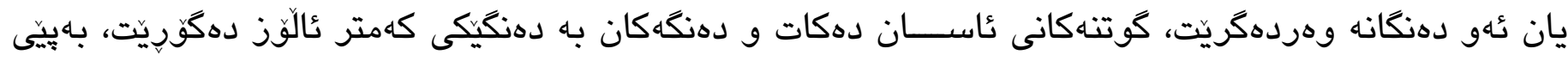

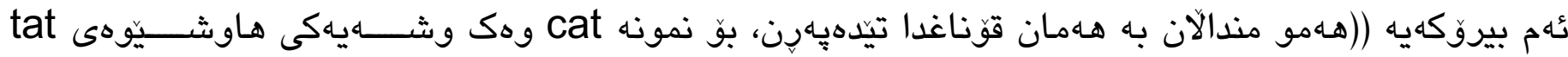

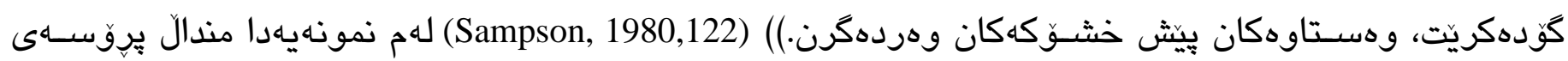

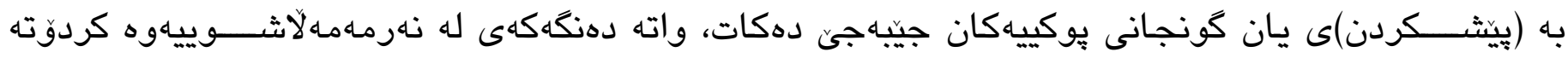

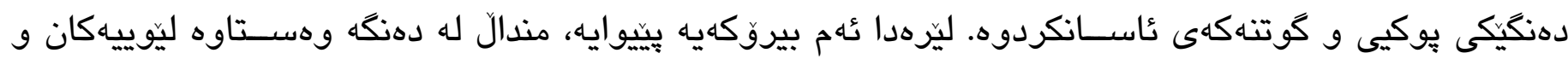

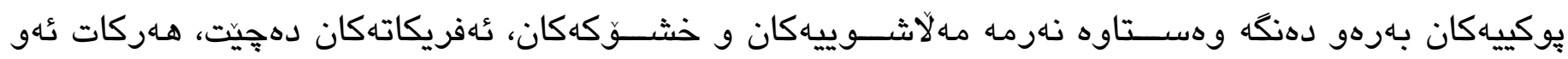

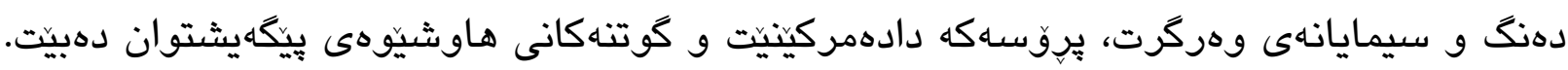

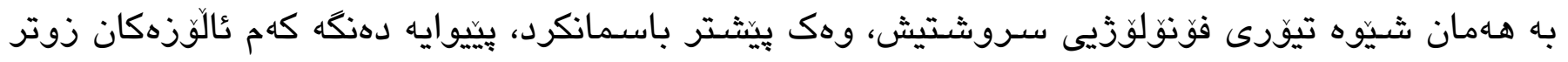

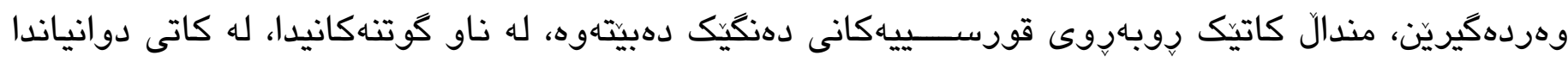

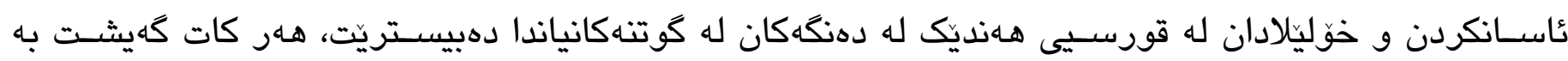

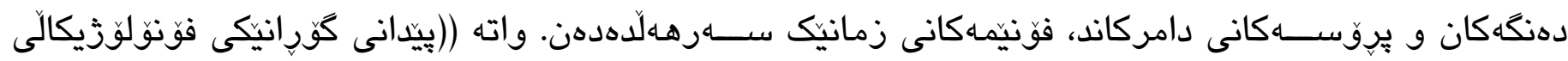

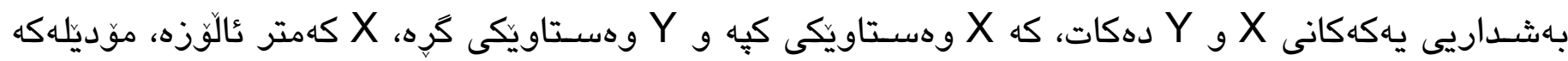

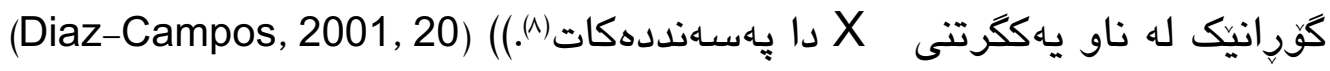

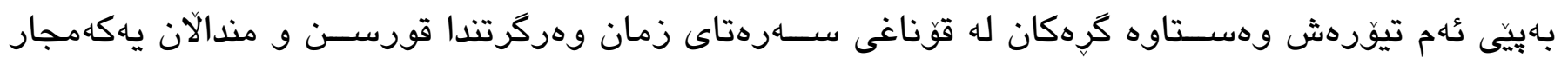

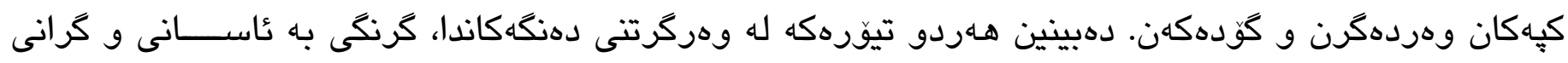

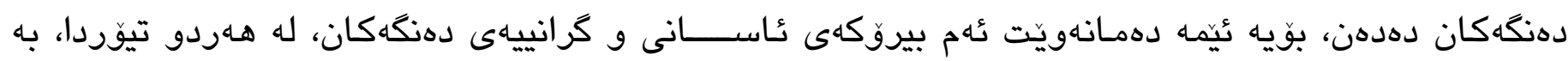

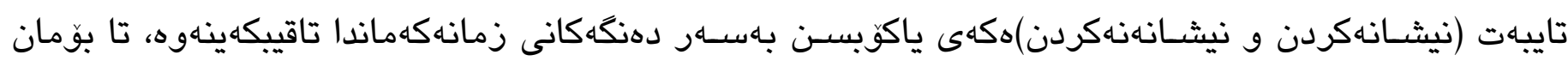

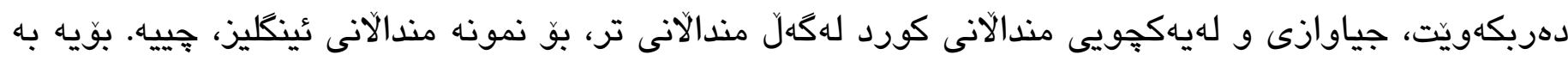

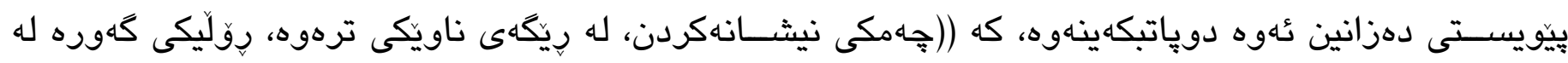

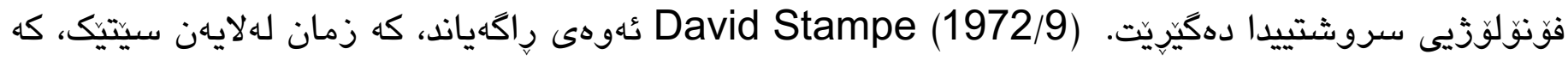

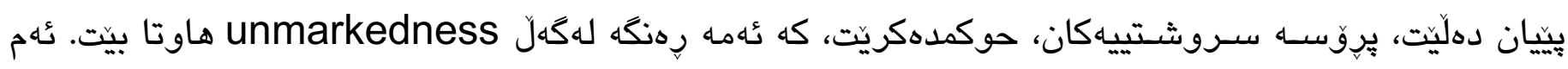

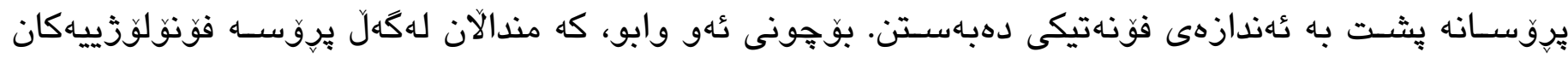

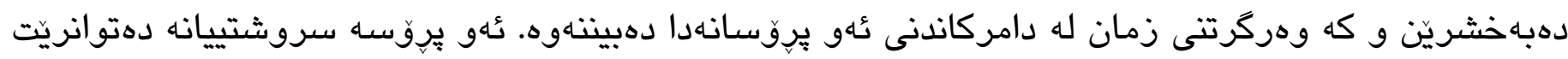

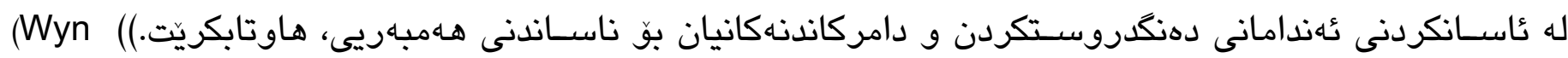
Johnson and Paula Reimers, 2010, 57)

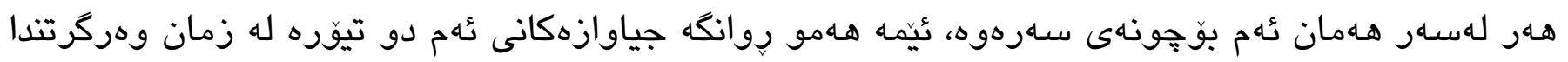

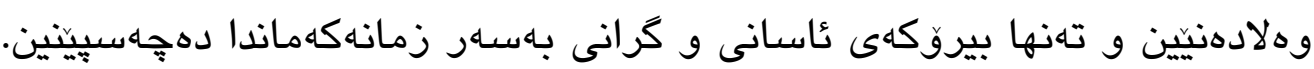

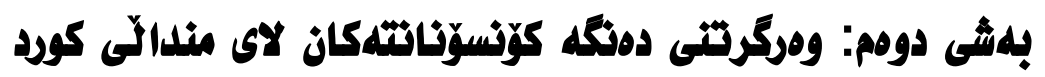

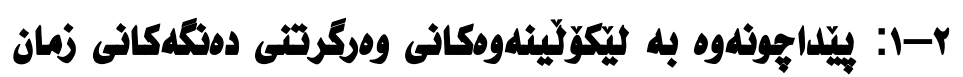

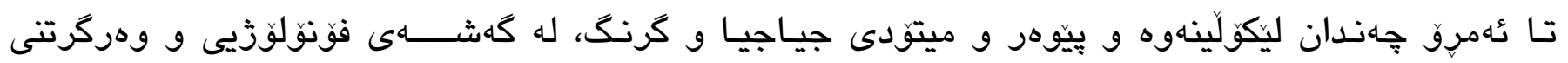

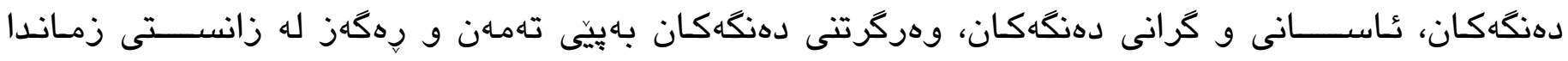
سـانريانههلَّاوه. 


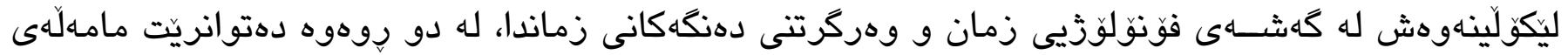

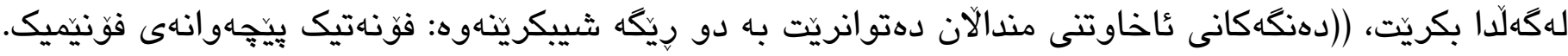

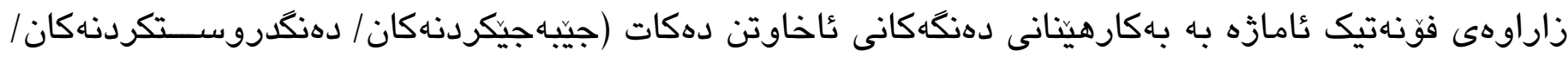

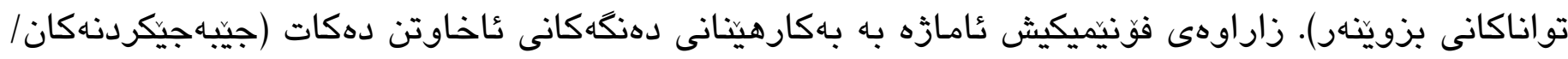

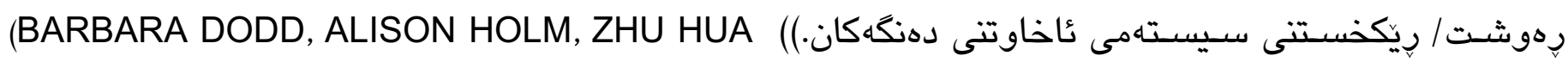
and SHARON CROSBIE, 2003, 618)

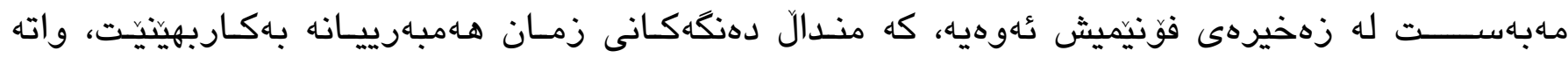

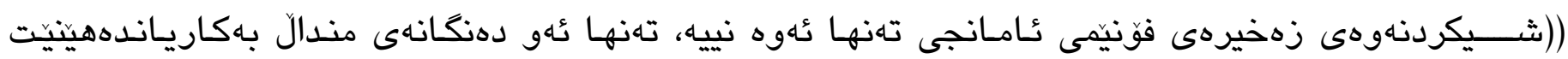

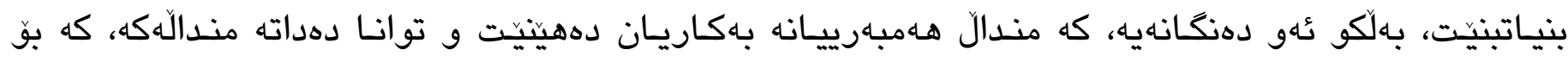

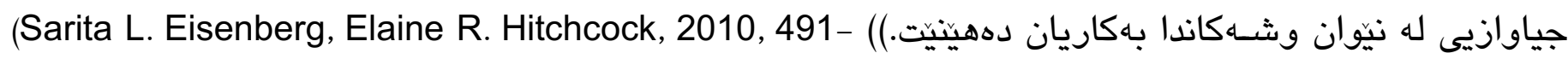
493)

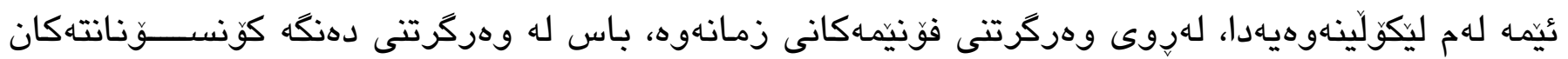

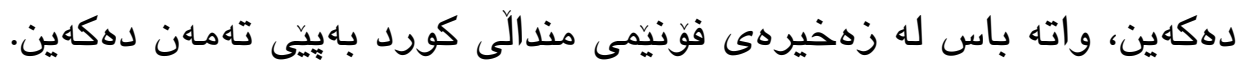

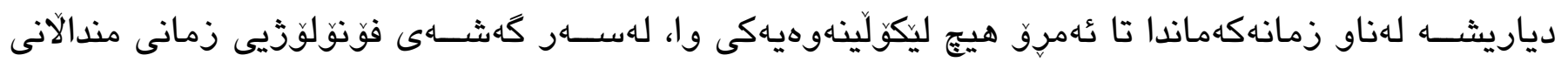

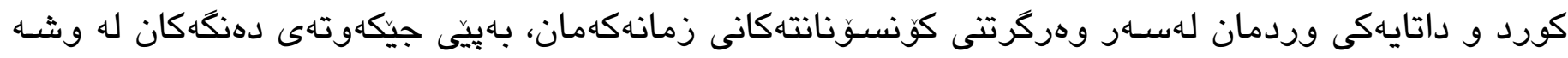

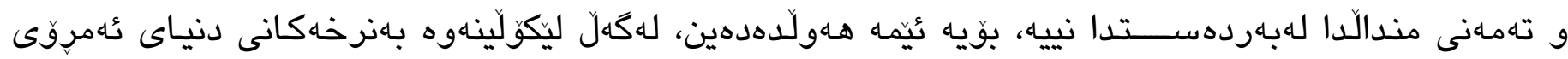

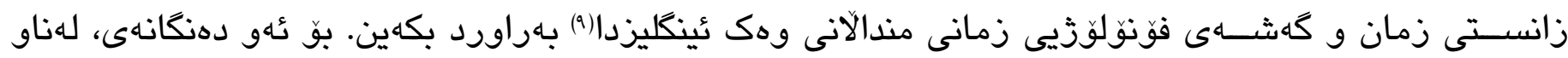

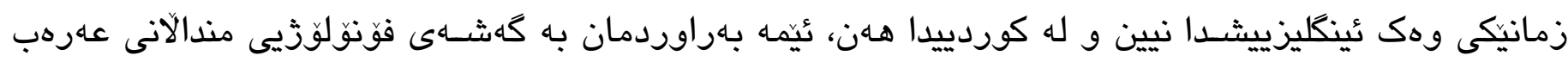

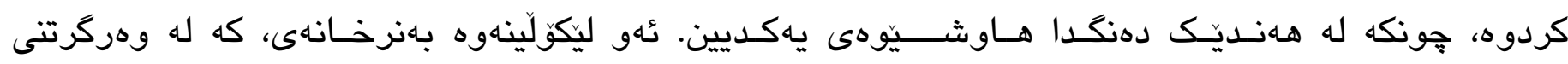

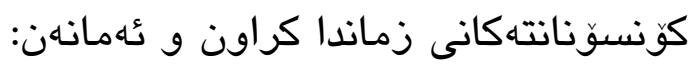

(Templin, 194), (Mousa M. Amayreh, Alice T. Dyson, 1998), (Smit, 1986) (Elizabeth M.Prather, Dona Lee Hedrick, Carolyn A. Kern, 1990-1991), (Ann Bosma Smit, Linda Hand, J.Joseph Freilinger, John E. Bernthal, Ann Bird 1990), (BARBARA DODD, ALISON HOLM, ZHU HUA and SHARON CROSBIE, 2003)

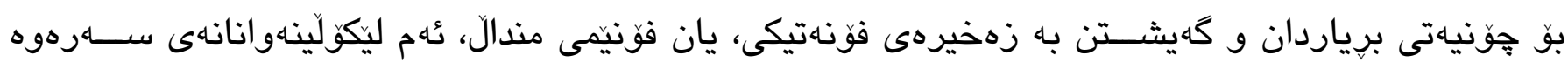

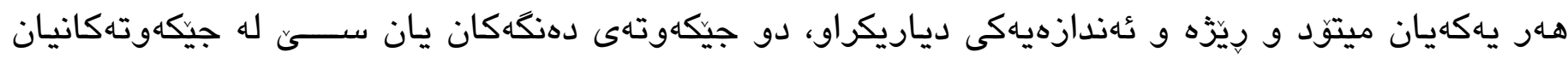

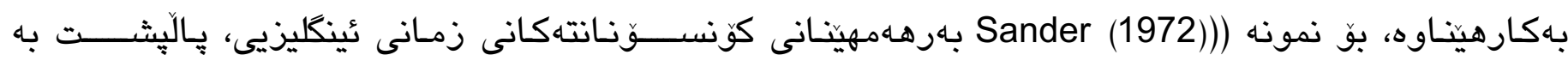

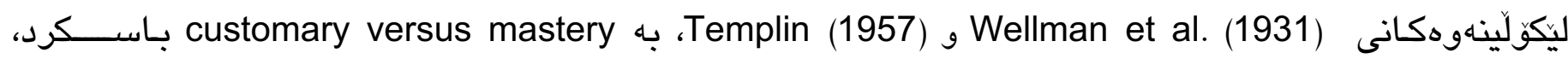

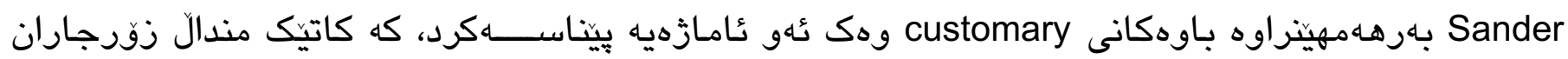

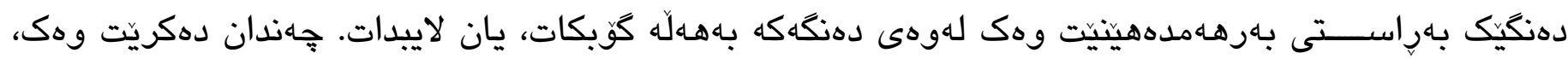

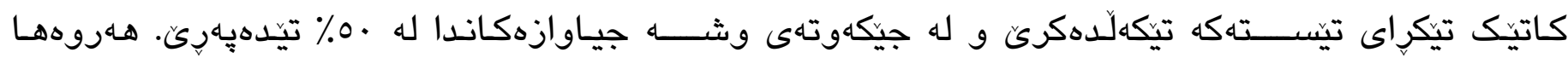

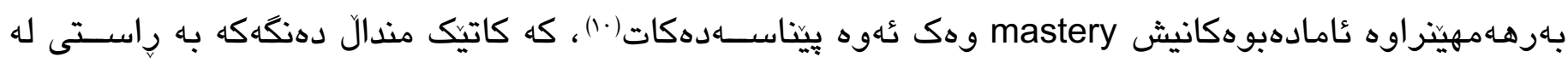

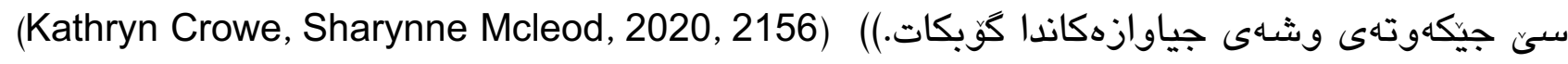




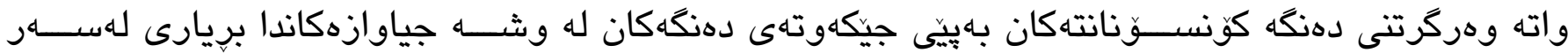

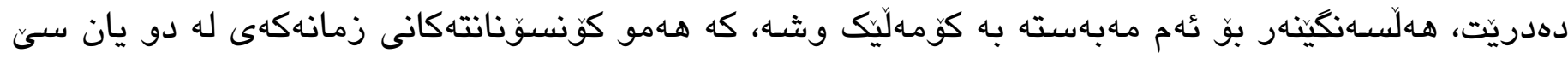

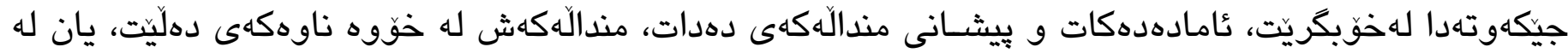

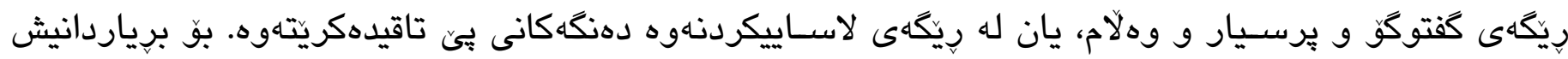

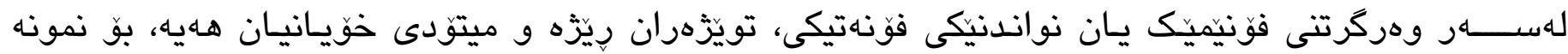

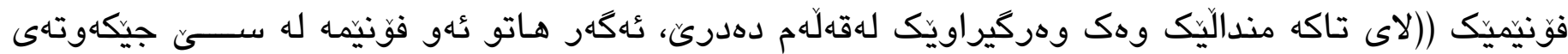

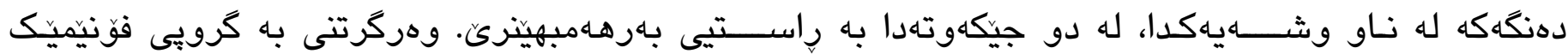

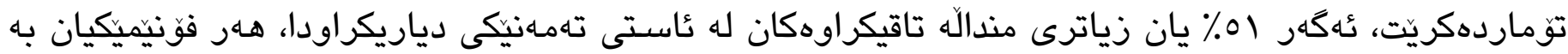

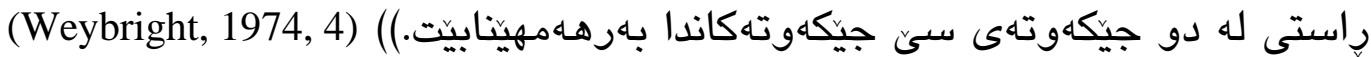

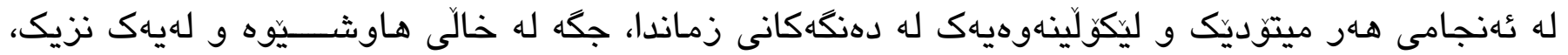

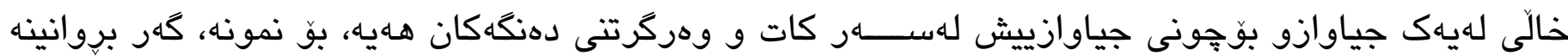

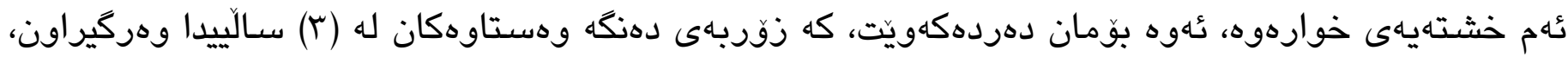

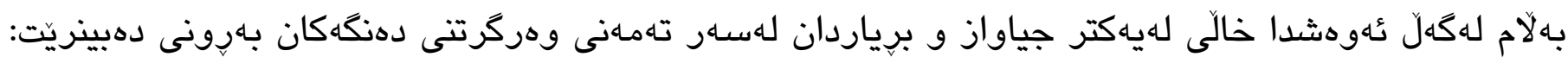

\begin{tabular}{|c|c|c|c|c|c|c|c|c|c|c|}
\hline $\begin{array}{c}\text { Templin } \\
\text { (1957) }\end{array}$ & $\begin{array}{c}\text { Anthony } \\
\text { et al. } \\
\text { (1971) }\end{array}$ & $\begin{array}{c}\text { Paynter } \\
\text { et al. } \\
\text { (1974) }\end{array}$ & $\begin{array}{c}\text { Prather } \\
\text { et al } \\
(1975)\end{array}$ & $\begin{array}{c}\text { Arlt and } \\
\text { Goodban } \\
(1976)\end{array}$ & $\begin{array}{c}\text { Kilminster } \\
\text { and Laird } \\
(1978)\end{array}$ & $\begin{array}{l}\text { Chirlian } \\
\text { and } \\
\text { Sharply } \\
(1982)\end{array}$ & $\begin{array}{l}\text { Smit } \\
\text { et al. } \\
(1990)\end{array}$ & $\begin{array}{c}\text { Smit et } \\
\text { al } \\
(1990)\end{array}$ & $\begin{array}{l}\text { Dodd, } \\
\text { holm at } \\
\text { al. } \\
(2003)\end{array}$ & \\
\hline \multirow[t]{5}{*}{$\begin{array}{l}75 \% \\
\text { United } \\
\text { States }\end{array}$} & $\begin{array}{l}90 \% \\
\text { Scotland }\end{array}$ & $\begin{array}{l}2 ; 0-2 ; 6 \\
90 \% \\
\text { United } \\
\text { States }\end{array}$ & $\begin{array}{l}2 ; 0-4 ; 0 \\
\\
75 \% \\
\text { United } \\
\text { States }\end{array}$ & $\begin{array}{l}3 ; 0-6 ; 0 \\
75 \% \\
\text { United } \\
\text { States }\end{array}$ & $\begin{array}{l}3 ; 0-9 ; 0 \\
75 \% \\
\text { Australia }\end{array}$ & $\begin{array}{l}2 ; 0-9 ; 0 \\
75 \% \\
\text { Australia }\end{array}$ & $\begin{array}{l}3 ; 0- \\
9 ; 0 \\
\text { males } \\
75 \% \\
\text { United } \\
\text { States }\end{array}$ & $\begin{array}{l}3 ; 0-9 ; 0 \\
\text { females } \\
75 \% \\
\text { United } \\
\text { States }\end{array}$ & $\begin{array}{l}3 ; 0-6 ; 11 \\
90 \% \\
\text { England } \\
\text { and } \\
\text { Australia }\end{array}$ & تُّهمهندكان \\
\hline & & h, w & $\begin{array}{c}m, \quad n, \\
y, h, p\end{array}$ & & & $\mathrm{~m}, \mathrm{n}, \mathrm{h}$ & & & & $2 ; 0$ \\
\hline & & & j, d, k, & & & & & & & $2 ; 4$ \\
\hline & & $\begin{array}{r}\mathrm{p}, \mathrm{m}, \mathrm{t}, \\
\mathrm{m}\end{array}$ & & & & $\begin{array}{r}P, y, w, \\
d, g\end{array}$ & & & & $2 ; 6$ \\
\hline & & & $\mathrm{w}, \mathrm{b}, \mathrm{t}$ & & & & & & & $2 ; 8$ \\
\hline \multirow[t]{4}{*}{$\begin{array}{r}\mathrm{m}, \mathrm{n}, \mathrm{y}, \\
\mathrm{p}, \mathrm{f}, \mathrm{h}, \\
\mathrm{w},\end{array}$} & $\begin{array}{r}\mathrm{p}, \mathrm{b}, \mathrm{m}, \\
\mathrm{t}, \mathrm{d}, \mathrm{n}, \\
\mathrm{w}, \mathrm{j}\end{array}$ & & $\mathrm{g}, \mathrm{s}$ & $\begin{array}{l}\mathrm{p}, \mathrm{b}, \mathrm{t}, \mathrm{d}, \\
\mathrm{k}, \mathrm{g}, \mathrm{m}, \\
\mathrm{n}, \mathrm{y}, \mathrm{h}, \mathrm{f}, \\
\mathrm{w}\end{array}$ & $\begin{array}{l}P, b, m, n, \\
\text { y, h, w, j, t, } \\
\text { d, k, g, } 3^{*}\end{array}$ & $\mathrm{~J}, \mathrm{k}, \mathrm{f}, \int$ & $\begin{array}{l}m, \quad n, \\
h, \quad w, \\
p, b, t, \\
d, k, g\end{array}$ & $\begin{array}{l}\mathrm{m}, \mathrm{n}, \mathrm{h}, \\
\mathrm{w}, \mathrm{p}, \mathrm{b}, \\
\mathrm{t}, \mathrm{d}, \mathrm{k}, \\
\mathrm{g}, \mathrm{f}, \mathrm{s}^{*}\end{array}$ & $\begin{array}{l}\mathrm{P}, \mathrm{b}, \mathrm{t}, \mathrm{d}, \\
\mathrm{k}, \mathrm{g}, \mathrm{m}, \\
\mathrm{n}, \mathrm{y}, \mathrm{f}, \mathrm{s}, \\
\mathrm{z}, \mathrm{h}, \mathrm{w}, \mathrm{l} \\
\mathrm{j}\end{array}$ & $3 ; 0$ \\
\hline & & & $1, \mathrm{r}$ & & & & & & & $3 ; 4$ \\
\hline & $\begin{array}{r}\mathrm{k}, \mathrm{g}, \mathrm{\eta}, \mathrm{f}, \\
\mathrm{v}, \mathrm{h}\end{array}$ & & & V & f & $b, t, \mathfrak{t}, \mathrm{d} s$ & $\mathrm{f}, \mathrm{j}$ & $\mathrm{J}$ & tf & $3 ; 6$ \\
\hline & & & $\int, \mathfrak{t}$ & & & & & & & $3 ; 8$ \\
\hline $\begin{array}{r}\mathrm{k}, \mathrm{b}, \mathrm{d}, \\
\mathrm{g}, \mathrm{r}\end{array}$ & & & ð, 3 & $\begin{array}{r}\mathrm{s}, \mathrm{z}, \mathrm{3}, \mathrm{t}, \\
\mathrm{d}, 1\end{array}$ & $1, \int, \mathfrak{t}$ & $\mathrm{L}, 3, \mathrm{~s}$ & ds & $\mathrm{V}$, б, $\underset{\mathrm{g}}{\mathrm{f}}$ & 3, ds & $4 ; 0$ \\
\hline \multirow[t]{3}{*}{$S, \int, \mathfrak{t}$} & 1 & & $\mathrm{~V}, \Theta, \mathrm{z}$ & $\int$ & ds, s*, $z^{*}$ & & V & ds, $1^{*}$ & & $4 ; 6$ \\
\hline & & & ds & $\Theta$, ə, r & $\mathrm{r}$ & $\mathrm{r}$ & $S^{*}, \int, \mathfrak{f}$ & $\mathrm{Z}^{*}$ & $\int$ & $5 ; 0$ \\
\hline & $\mathrm{S}, \mathrm{z}, \int, 3$ & & & & & & ə, r & $\eta, \Theta$ & & $5 ; 6$ \\
\hline \multirow[t]{2}{*}{$\mathrm{t}, \Theta, \mathrm{v}, \mathrm{l}$} & & & & & V & & $\begin{array}{l}\mathrm{y}, \quad \Theta, \\
\mathrm{z}, 1\end{array}$ & $r$ & $\mathrm{r}$ & $6 ; 0$ \\
\hline & $\Theta$, ð, † & & & & & & & & & $6 ; 6$ \\
\hline
\end{tabular}




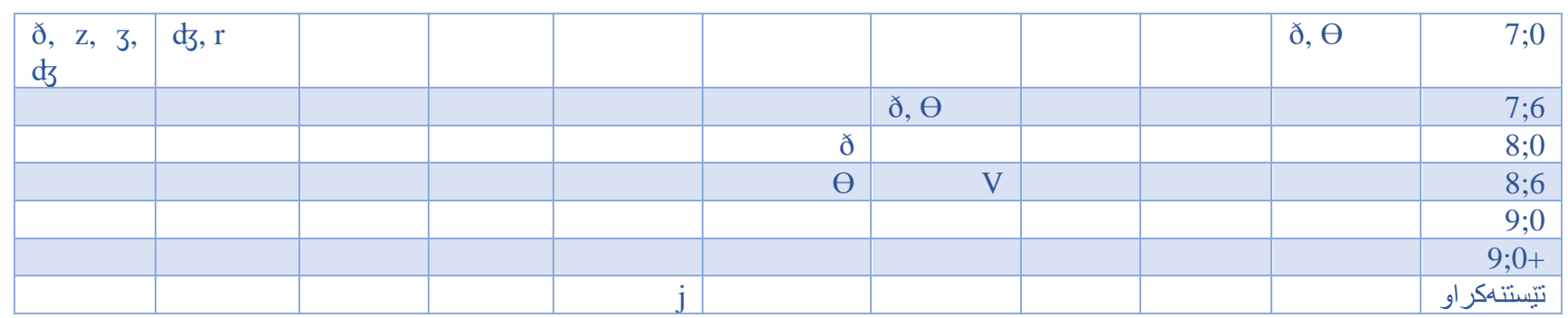

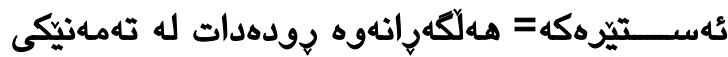

خشــــــ زمّاره (Y): وهركرتنى دهنكهكانى زمانى يُينكليزيى.

(John E. Bernthal, Nicholas W. Bankson, Peter Flipsen Jr, 2016, 76-77)

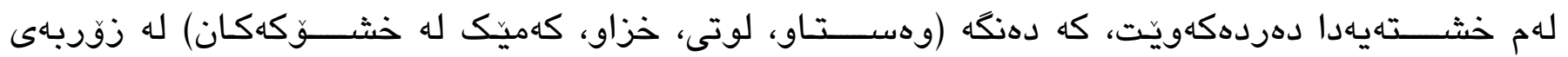

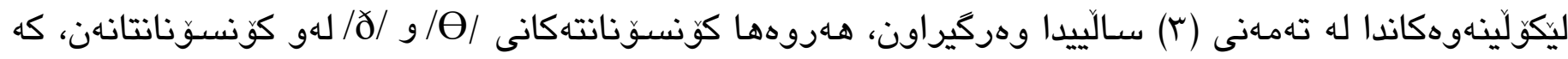

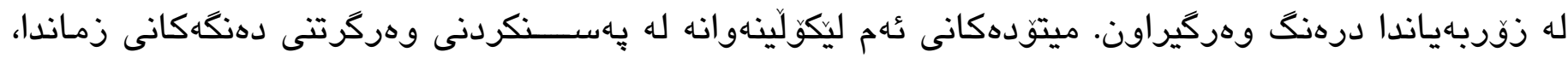

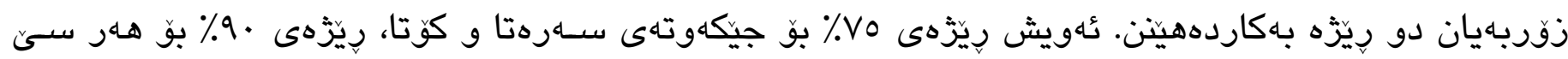

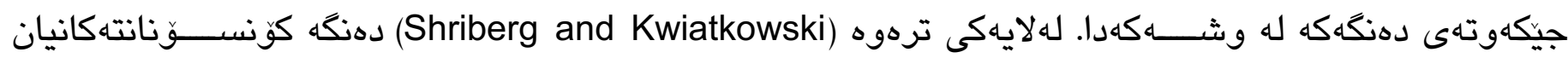

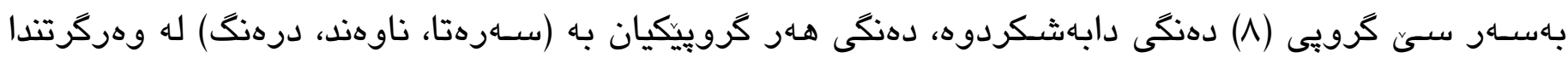

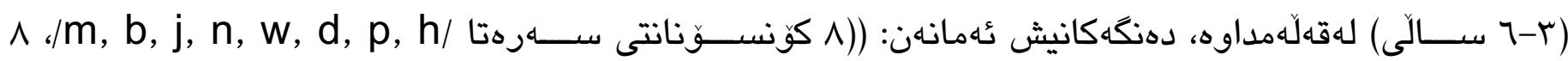

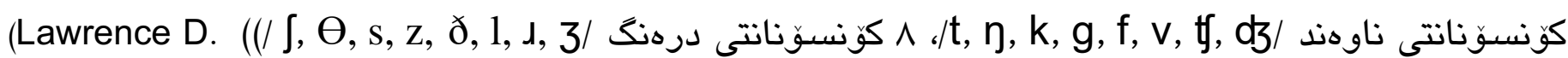
Shriberg, Joan Kwiatkowski, 1994, 1105)

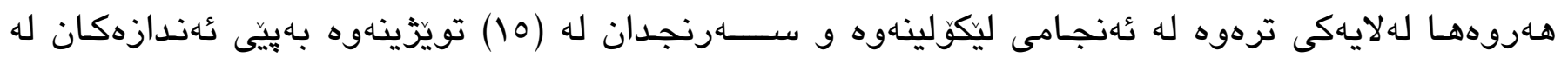

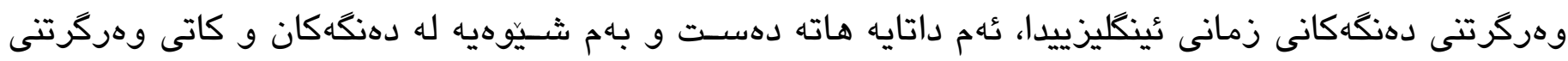

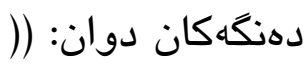

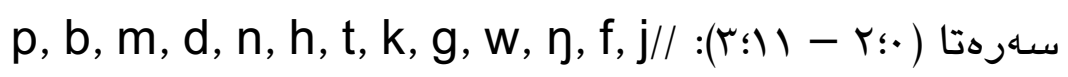

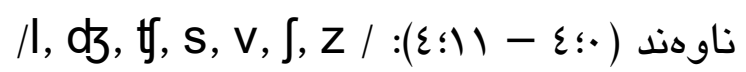

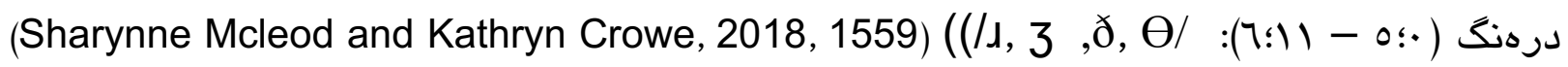

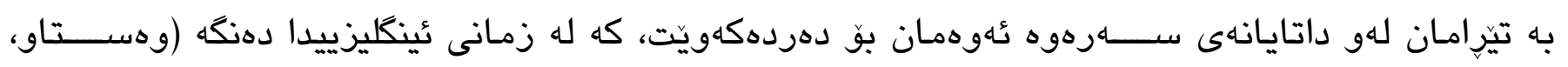

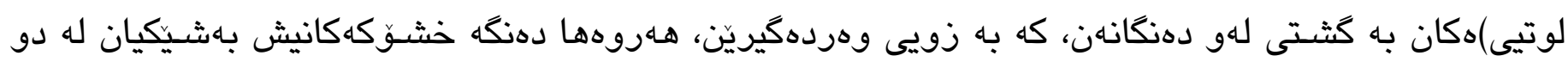

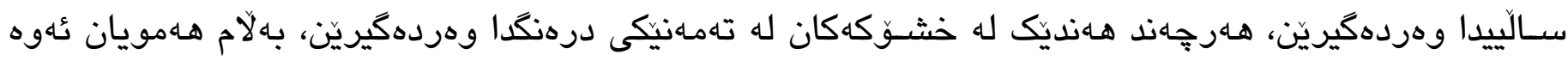

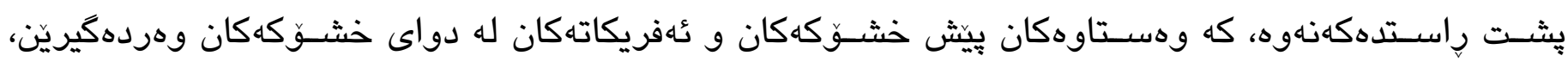

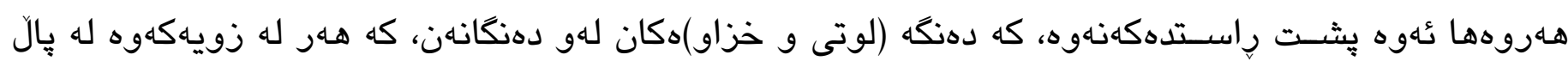
وهستاوهكان وهردهكيرين.

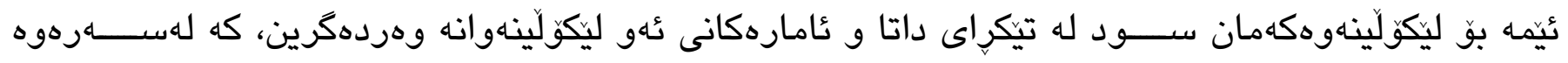

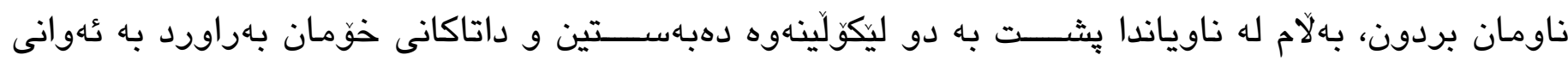

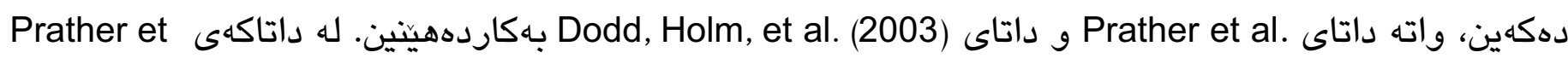

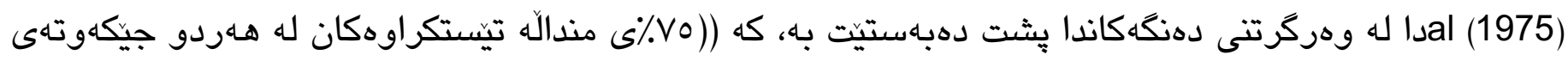

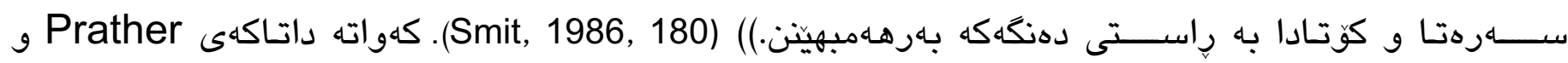

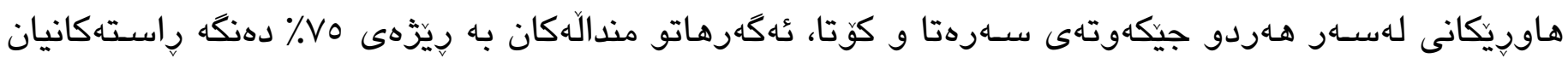




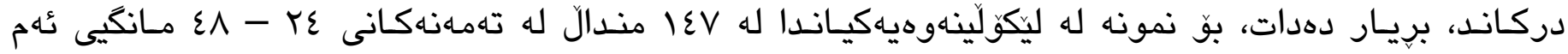

\begin{tabular}{|c|c|c|c|}
\hline تهمـن & دهنگ & تهمهن & دهنگ \\
\hline$r$ & G & $r$ & $\mathrm{~m}$ \\
\hline r & S & r & $n$ \\
\hline$\S r ! \varepsilon$ & $\mathrm{R}$ & r & $\mathrm{h}$ \\
\hline$\S$ & I & r & $p$ \\
\hline ऍ & $\int$ & $r$ & $\eta$ \\
\hline r؛^ & t5 & $r ! \varepsilon$ & f \\
\hline$\varepsilon$ & 3 & $r ! \varepsilon$ & j \\
\hline${ }^{\circ}+\varepsilon$ & d3 & r!६ & $\mathrm{k}$ \\
\hline${ }^{\circ}+\varepsilon$ & V & $r ! \varepsilon$ & $d$ \\
\hline \multirow[t]{3}{*}{${ }^{\circ}+\varepsilon$} & Z & $r \leqq \wedge$ & w \\
\hline & & $r: \Lambda$ & b \\
\hline & & Y`A & $t$ \\
\hline
\end{tabular}

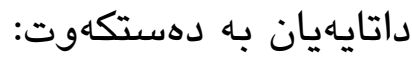

خشتهى زماره (r): وهركرتنى كونسوّناتتكانى زمان بهيني ميتودهكهى (Prather e atl)

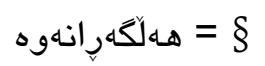

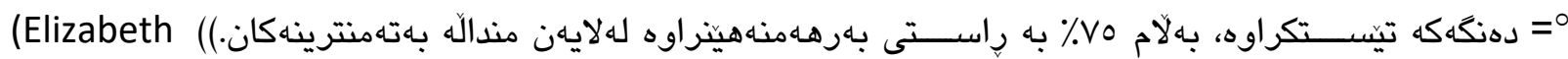

M.Prather, Dona Lee Hedrick, Carolyn A. Kern, 1990-1991, 99)

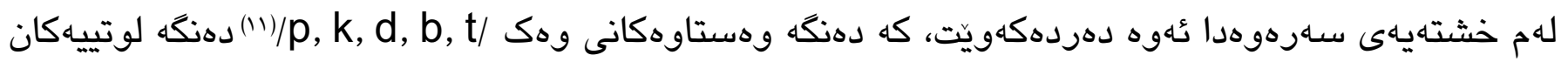

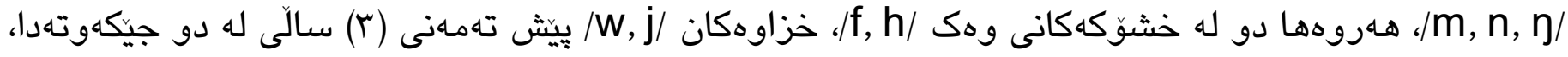

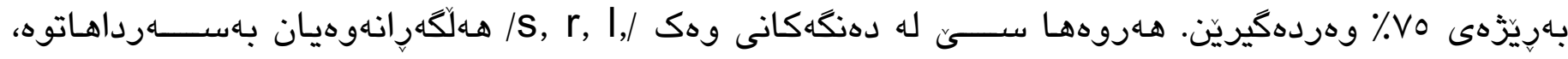

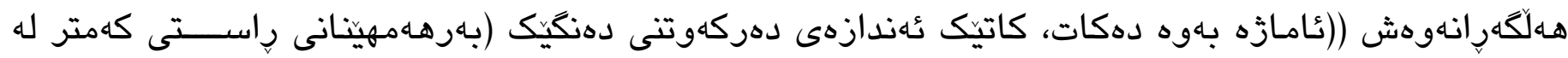

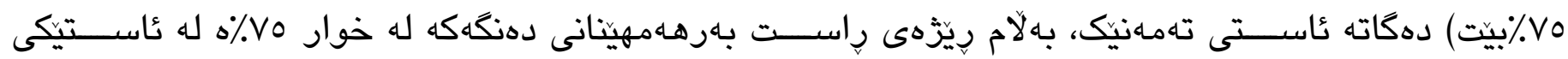

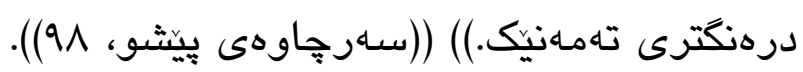

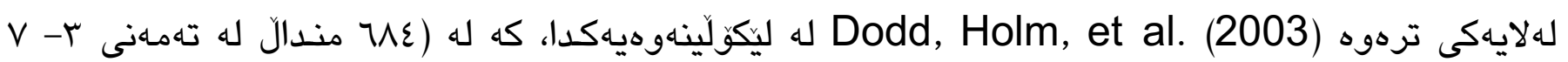

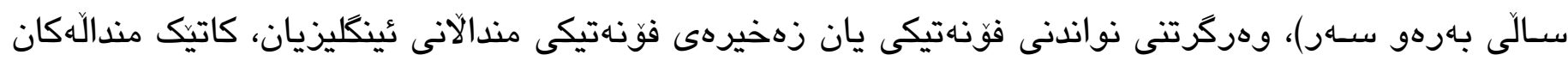

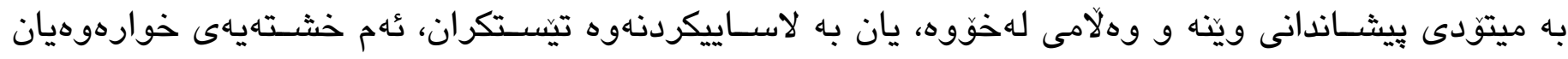

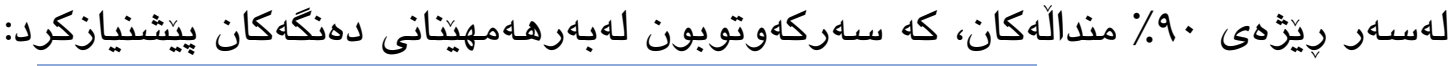

\begin{tabular}{|c|c|c|}
\hline ت عاماده & & تهمهن \\
\hline $\begin{array}{l}\mathrm{p}, \mathrm{b}, \mathrm{t}, \mathrm{d}, \mathrm{k}, \mathrm{g}, \mathrm{m}, \\
\mathrm{n}, \mathrm{\eta}, \mathrm{f}, \mathrm{v}, \mathrm{s}, \mathrm{z}, \mathrm{h}\end{array}$ & 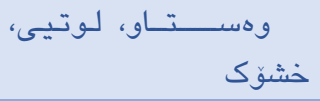 & • \\
\hline w, l-, j & نزيكبودكان & ד!r \\
\hline \multirow[t]{2}{*}{$\begin{array}{r}t \\
3 \\
d 3\end{array}$} & خُشهريكات & $\varepsilon ! 0-\varepsilon ! \bullet$ \\
\hline & & $\varepsilon ! 1)-\varepsilon ! 7$ \\
\hline $\int$ & خشوّك & $0 ! 0-0 !$ \\
\hline & & 7 \\
\hline 1 & نزيكبو & $7 ! 0-7 ! \cdot$ \\
\hline$\Theta$, Ә & خشوك & V بهرهو سهرهوه \\
\hline
\end{tabular}




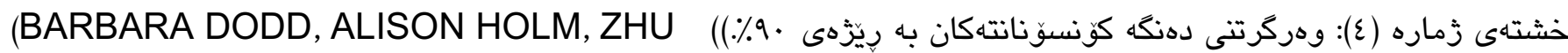

HUA and SHARON CROSBIE, 2003, 635)

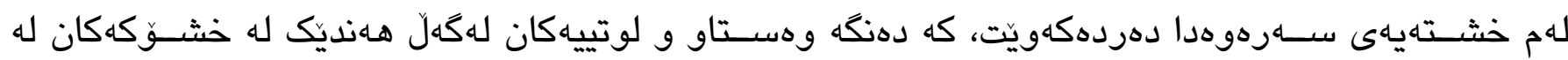

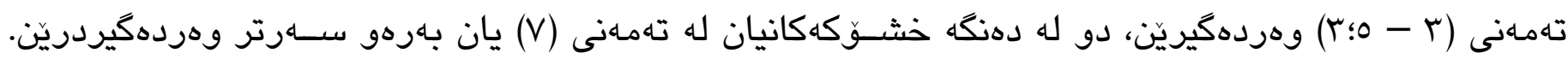

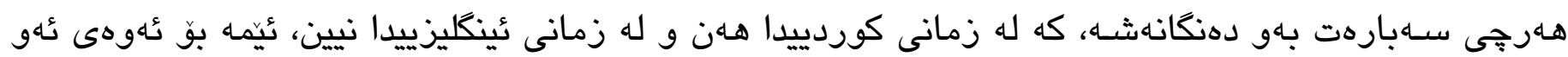

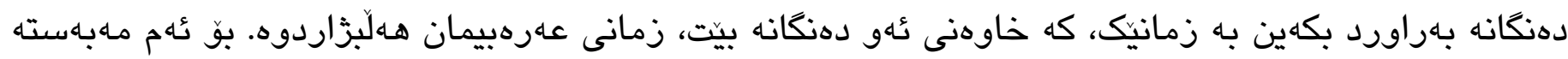

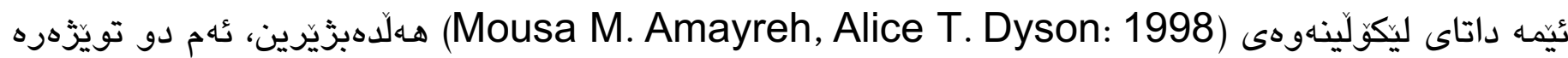

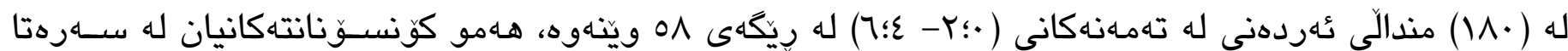

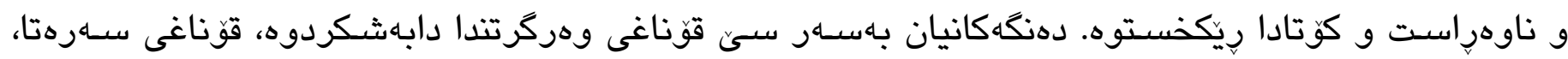

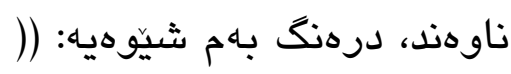

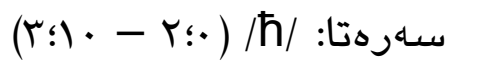

ناوهند: (

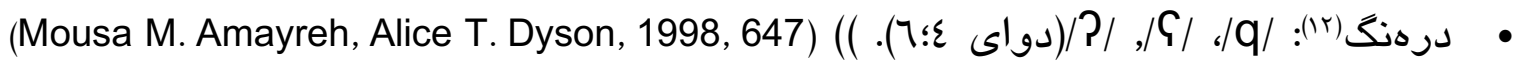

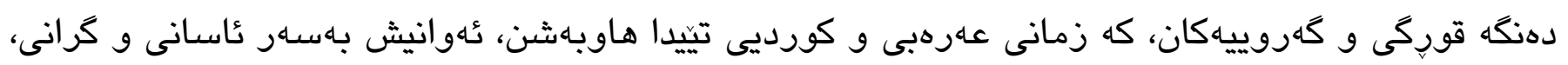

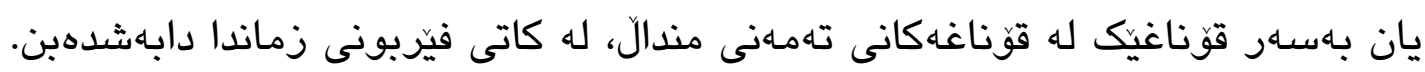

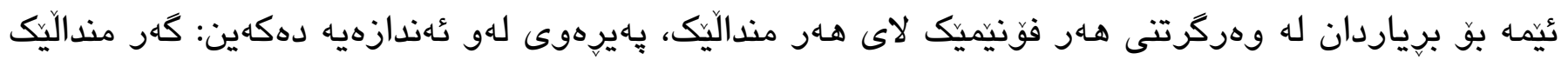

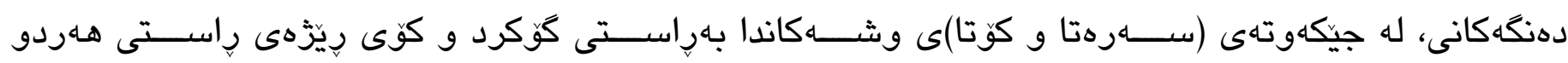

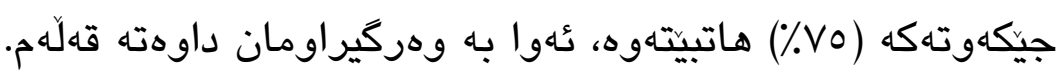

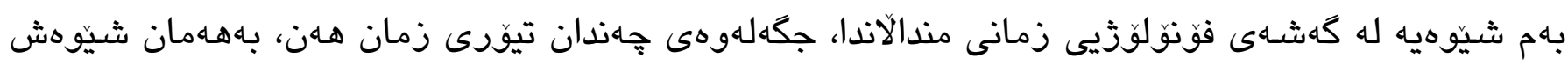

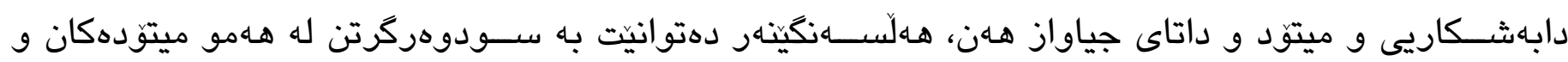

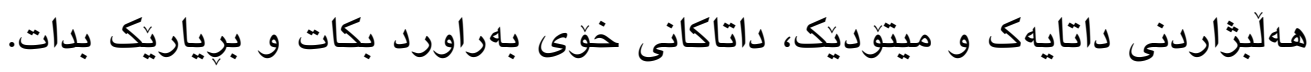

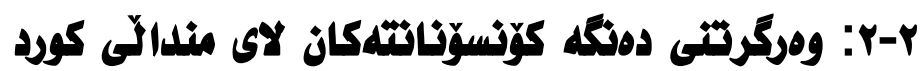

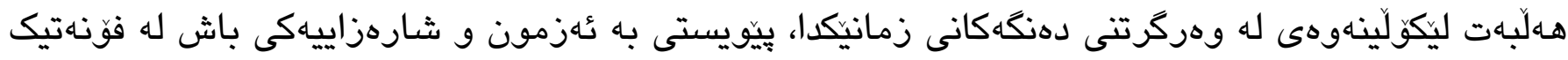

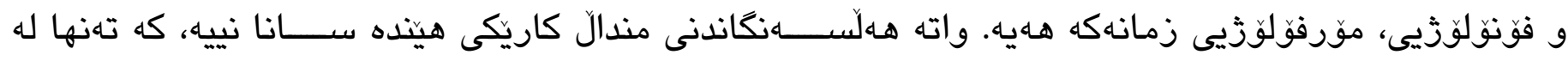

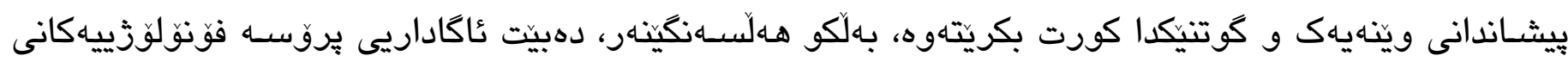

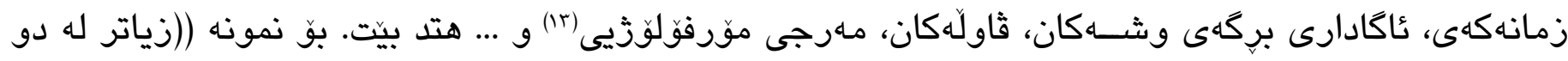

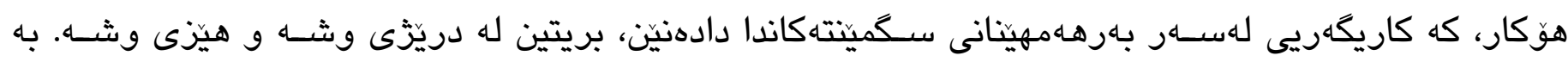

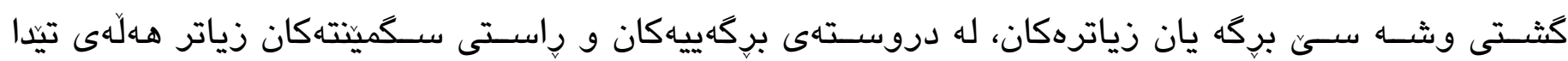

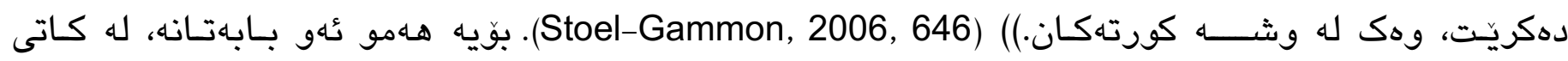

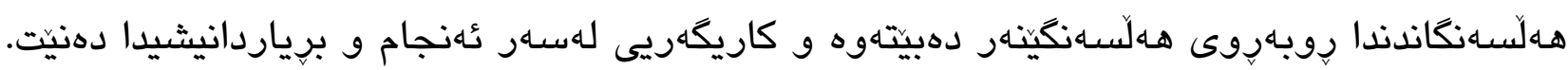

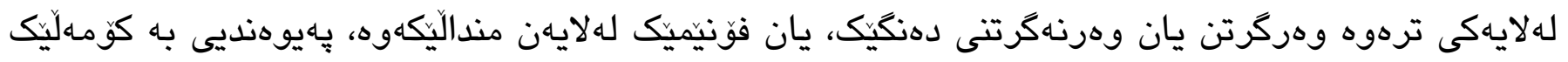

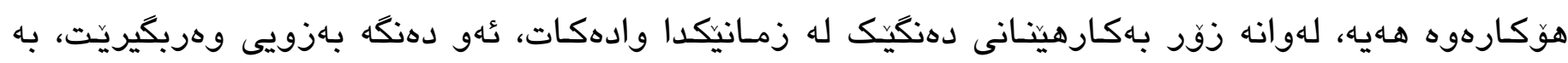

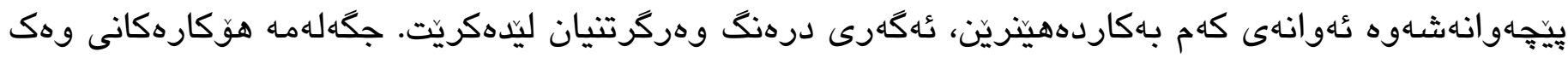




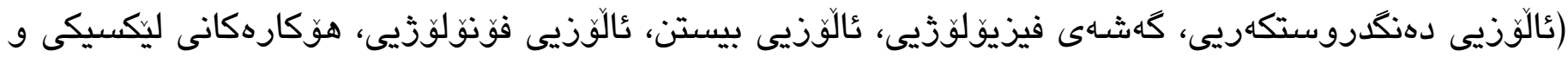

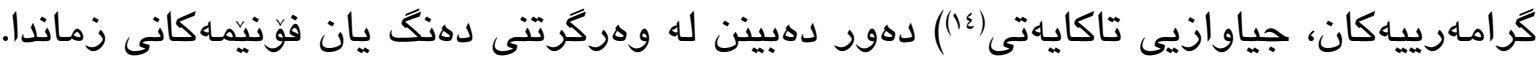

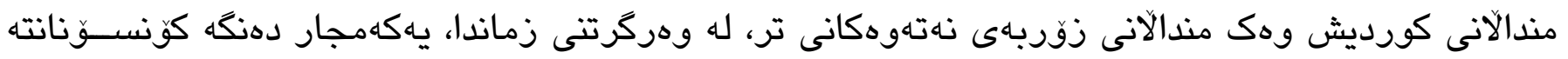

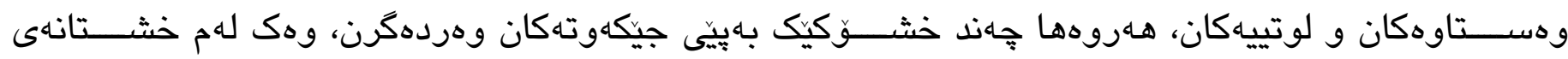

خشتهى زماره (0): زهخيرهى فؤنيمى (10) له تهمهنى (ع؛ (ب)

\begin{tabular}{|c|c|c|c|c|}
\hline $\begin{array}{r}\text { Final } \\
\text { كوتا }\end{array}$ & Medial ناوهراست & Initial سهرهتا & دهونيهتى & $\begin{array}{l}\text { (كور) } \\
\text { (كو) }\end{array}$ \\
\hline$t, q$ & $p, b, t, d, k$ & $\begin{array}{l}\mathrm{p}, \mathrm{b}, \mathrm{t}, \mathrm{d}, \mathrm{k}, \\
\mathrm{g}, ?\end{array}$ & وهستاو & \\
\hline \multirow[t]{2}{*}{$\mathrm{m}$} & $\mathrm{m}, \mathrm{n}$ & $\mathrm{m}$ & لوتيى & \\
\hline & $w, j$ & w & خزاو & \\
\hline \multirow[t]{3}{*}{$\int$} & $f, s, h, x$ & $\mathrm{~s}, \int, \mathrm{S}, \mathrm{h}$ & خشوّك & \\
\hline & dz & & ئهفريكات & \\
\hline & & I & لايى & \\
\hline r & $r$ & & للهروك & \\
\hline
\end{tabular}

خشتهى زماره (7): زهخيرهى فُونيمى له تهمانى (I!r)

\begin{tabular}{|c|c|c|c|c|}
\hline كوتا Final & ناوهراست Medial & سهرهتا Initial & دهورنهامهينانى & $\begin{array}{c}r \text { (كوي) } \\
\text { (ك) }\end{array}$ \\
\hline $\mathrm{t}, \mathrm{k}$ & $b, p, t, d, k, g, q$ & $\begin{array}{l}b, p, d, k, g, \\
q, ?\end{array}$ & ودستاو & \\
\hline \multirow[t]{2}{*}{$\mathrm{m}, \mathrm{n}$} & $\mathrm{m}, \mathrm{n}$ & $\mathrm{m}, \mathrm{n}$ & لوتيى & \\
\hline & $w, j$ & & خزاو & \\
\hline \multirow[t]{3}{*}{$x, \theta$, ठ } & $v, \int, \quad \theta, \quad$ ठ & $\mathrm{x}, \mathrm{h}, \int, \quad \theta, \quad \delta$ & خشوك & \\
\hline & ds & d3 & ئهفريكات & \\
\hline & 1,4 & & لايى & \\
\hline$r$ & & & ل لدروك & \\
\hline
\end{tabular}


خشتهى زماره (V): زهخيرهى فونيْيمى له تهمهنى (·!)

\begin{tabular}{|c|c|c|c|c|}
\hline كوّتا Final & ناوهند Medial & سلهرهتا Initial & دهونيهتى & $\begin{array}{l}\text { (كور) } \\
\text { (كور }\end{array}$ \\
\hline$b, p, k$, & $\begin{array}{l}b, p, t, d, k \\
g\end{array}$ & $\begin{array}{l}b, p, t, d, k \text {, } \\
g, ?\end{array}$ & و وستاو & \\
\hline \multirow[t]{2}{*}{$m, n$} & $\mathrm{~m}, \mathrm{n}$ & $\mathrm{m}$ & لوتيى & \\
\hline & $w, j$ & j & خزاو & \\
\hline \multirow[t]{3}{*}{$\int$} & $f, v, s, \int, z, x$ & $f, s, \int, h, x$ & خشوّك & \\
\hline & & t5 & ئدفريكات & \\
\hline & 1,4 & & لايى & \\
\hline$r$ & $r, r$ & & للمروك & \\
\hline
\end{tabular}

خشتهى زماره (^): زهخيرهى فُونيمى له تهمهنى (ا؟r)

\begin{tabular}{|c|c|c|c|c|}
\hline كوَتا Final & ناوهند Medial & سدرهتا Initial سلا & بهورنيهنى & $\begin{array}{l}\text { (كور) } \\
\text { (كور }\end{array}$ \\
\hline $\begin{array}{l}b, p, t \\
d, k\end{array}$ & $\begin{array}{l}\mathrm{b}, \mathrm{p}, \mathrm{t}, \mathrm{d}, \mathrm{k}, \\
\mathrm{g}, \mathrm{q}\end{array}$ & $\begin{array}{l}b, p, t, d, k \text {, } \\
g, q, ?\end{array}$ & وهتناو & \\
\hline \multirow[t]{2}{*}{$\mathrm{m}, \mathrm{n}$} & $m, n$ & $\mathrm{~m}, \mathrm{n}$ & لوتيى & \\
\hline & $w, j$ & j & خزاو & \\
\hline$f, \theta$, ठ & $\begin{array}{l}f, v, \theta, z, \\
\hbar, \varsigma, x\end{array}$ & $\begin{array}{l}f, s, z, \hbar, \\
\varsigma, h\end{array}$ & خشُوَ & \\
\hline \multirow[t]{2}{*}{ t5 } & & t5 & ئدفريكات & \\
\hline & & & لايى & \\
\hline r & $r$ & & للمروَى & \\
\hline
\end{tabular}


خشتهى زماره (9): زهخيرهى (17 ) فوَنيمى له تهمانى (V)

\begin{tabular}{|c|c|c|c|c|}
\hline $\begin{aligned} & \text { Sوتّا } \text { كوnal } \\
& \text { Final }\end{aligned}$ & ناوهراست Medial & $\begin{array}{l}\text { سلكرهتا } \\
\text { Initial }\end{array}$ & دهونيهتى دهنهينانى & 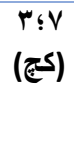 \\
\hline$p, t$ & $p, t, d$ & $\begin{array}{l}b, p, t, d, k \text {, } \\
g, ?\end{array}$ & و ومستاو & \\
\hline$m, n$ & $m, n$ & $\mathrm{~m}, \mathrm{n}$, & 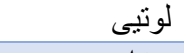 & \\
\hline & J & & خز او & \\
\hline $\begin{array}{l}s, z, \int \\
x,\end{array}$ & $f, v, s, z, \int$ & $s, z, x, \hbar$ & خشوك & \\
\hline t5 & $d 3, t 5$ & $d_{3}, t$ & ئهفريكات & \\
\hline I, & 1,4 & $\mathrm{I}$, & لايى & \\
\hline r & $r$ & & للهروَك & \\
\hline
\end{tabular}

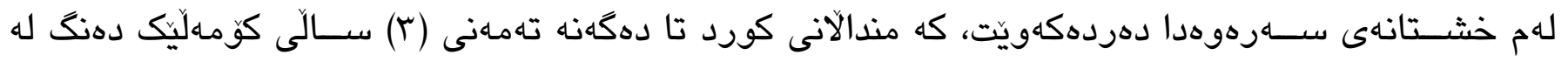

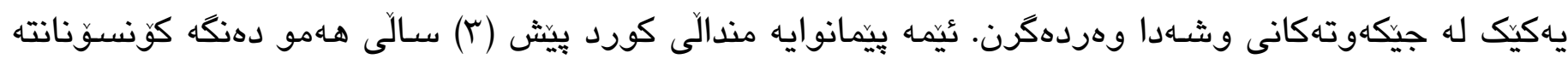

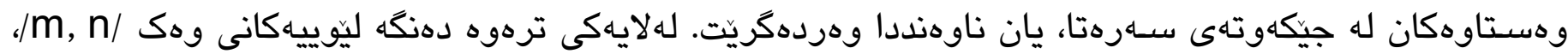

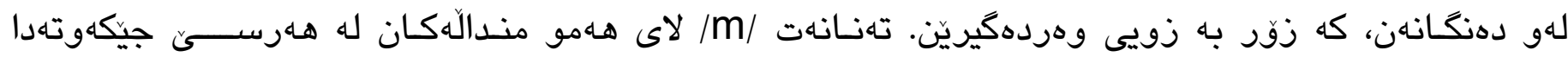
دهبينريّت (IV).

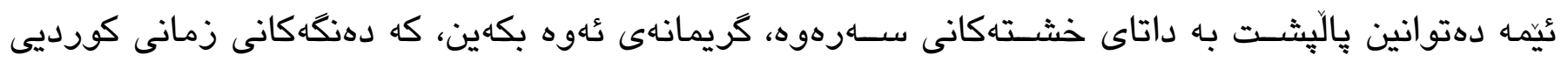

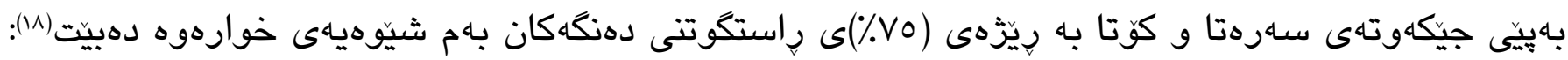

\begin{tabular}{|c|c|}
\hline 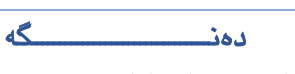 & تهمهن \\
\hline كونسوَنانتهكان & \\
\hline $\mathrm{t}$ & $r !)$ \\
\hline $\mathrm{m}$ & Y!) \\
\hline w & $Y ! 1$ \\
\hline$\S \int$ & Y!) \\
\hline $\mathrm{h}$ & $r !)$ \\
\hline ? & $Y !)$ \\
\hline$\S r$ & Y!) \\
\hline $\mathrm{n}$ & ६ \\
\hline $\mathrm{x}$ & $r ! \varepsilon$ \\
\hline$\theta$ & $r ! \varepsilon$ \\
\hline б & Y!६ \\
\hline b & Y؛A \\
\hline$p$ & Ys \\
\hline j & Y؛A \\
\hline$\S K$ & Y؛A \\
\hline d & r!) \\
\hline$\S t$ & r!) \\
\hline $\mathrm{f}$ & r!) \\
\hline I & $r: v$ \\
\hline
\end{tabular}

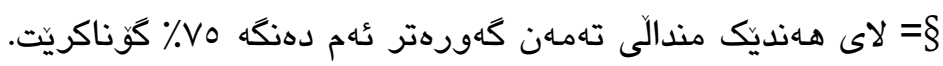

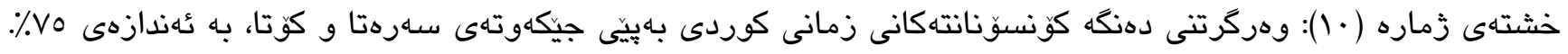




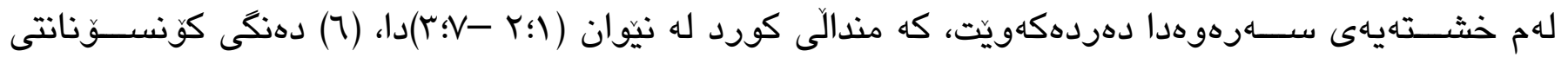

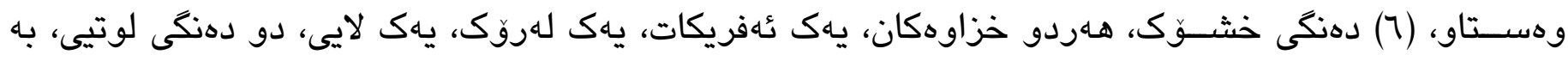
ييّى جيكهوتهى (سهردتا و كؤتا) وهردهكريت.

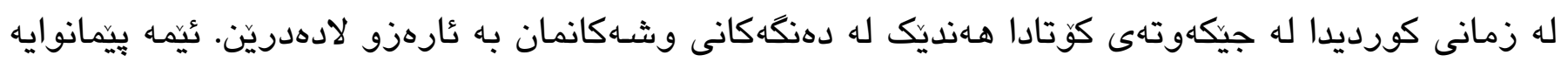

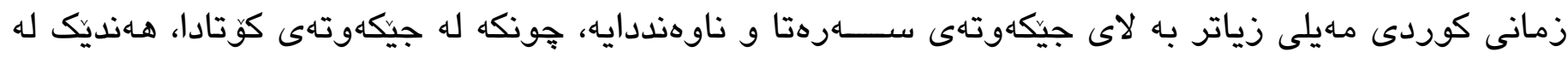

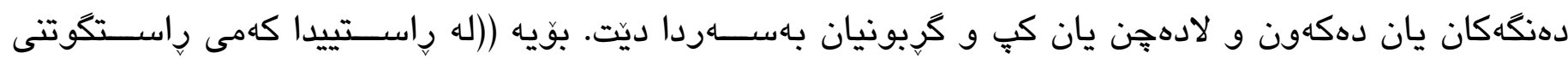

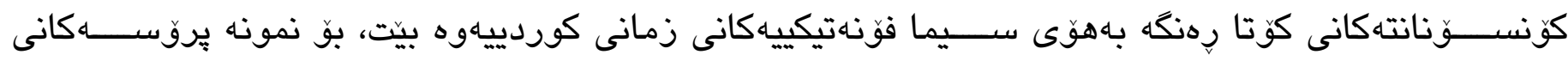

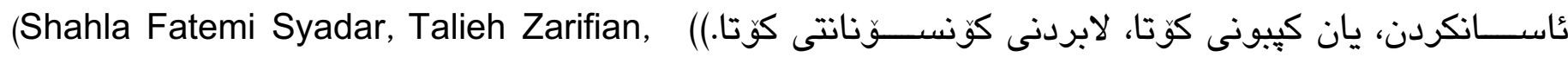
Yahya Modarresi, Muhamad Sediq Zahedi, Mona Ebrahimipur, Akbar Biglarian, 2018, 160)

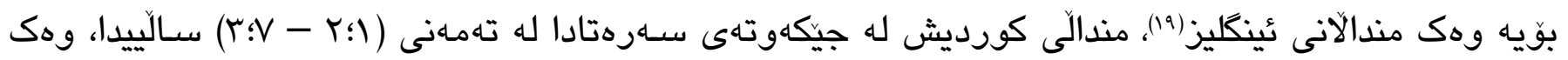

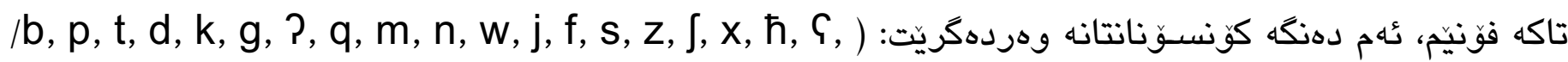
$h, d 3, t, l)$ له خشتهى زماره ( • ()دا دهردهكهويت، كه كوَنسوَنانته وهستاوهكان وهك فوَنيمهـكان، يهكهم دهسته دهبن كه

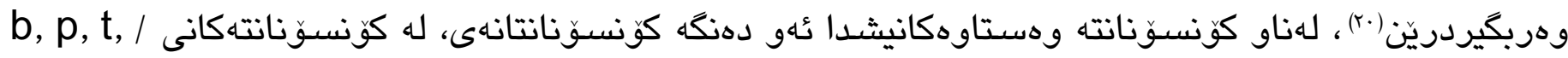

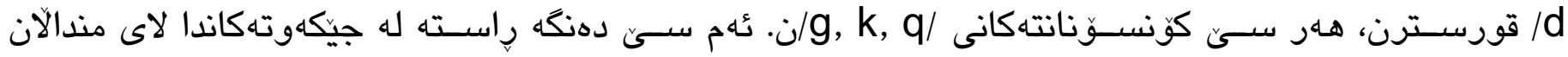

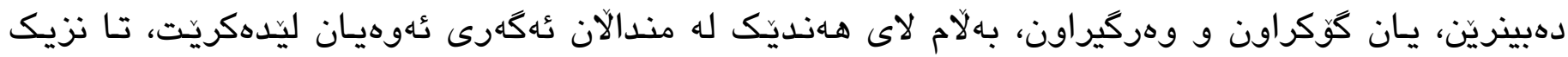

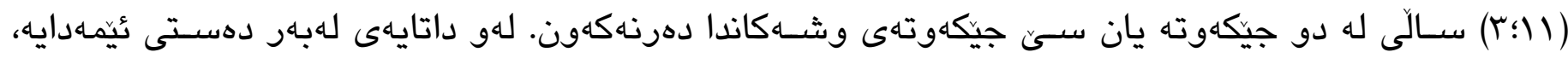

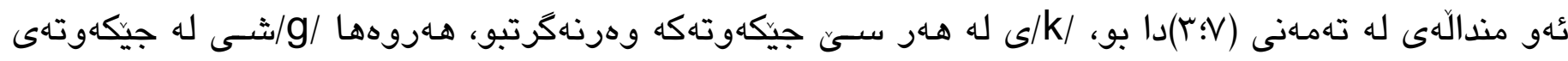

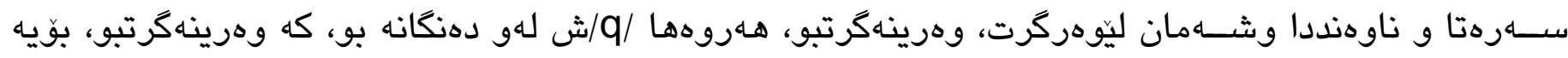

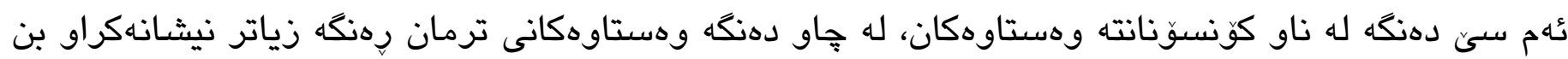

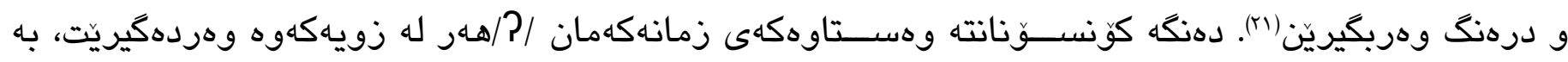

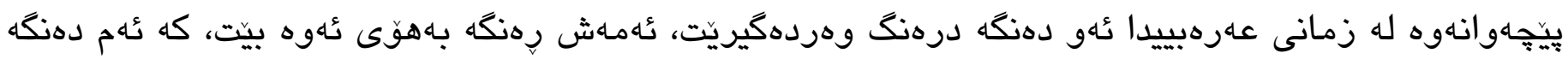

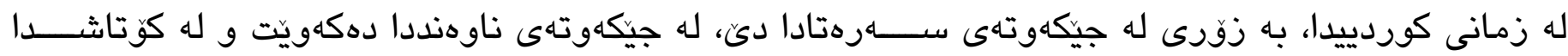

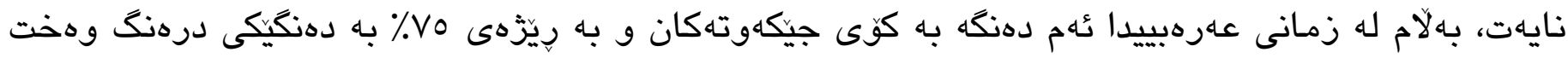
لـهقلّهم دراوه.

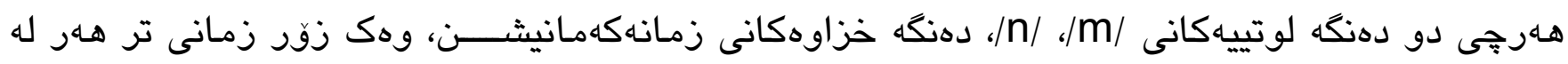

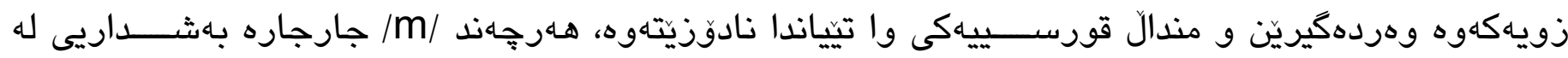

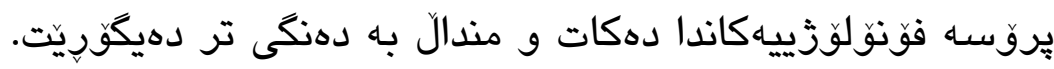

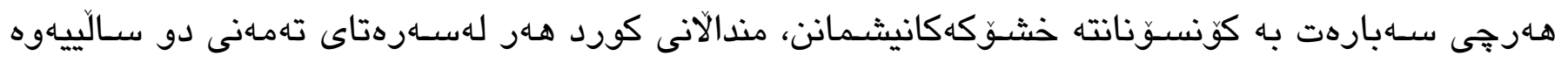

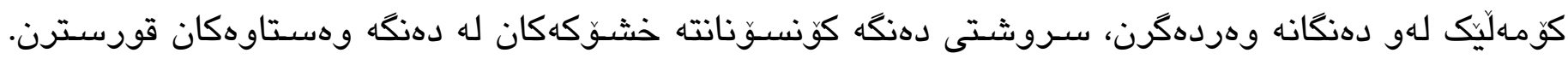

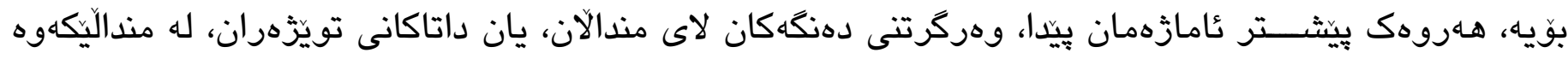

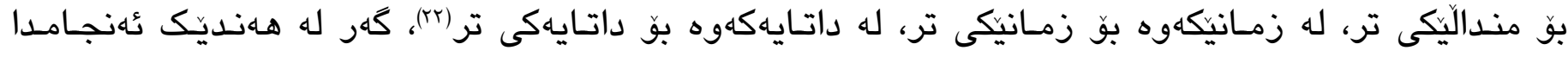

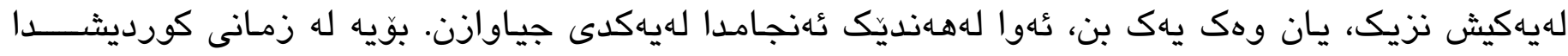

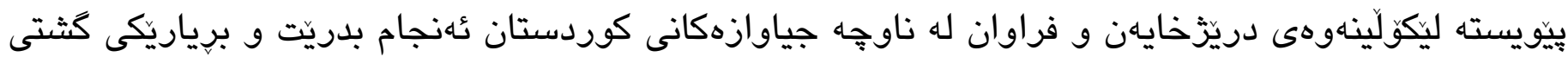
لهبارهيانهوه بلدريّت. 


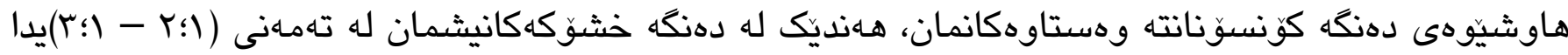

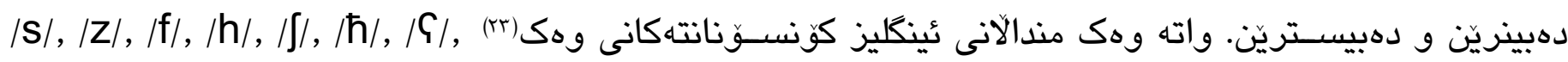

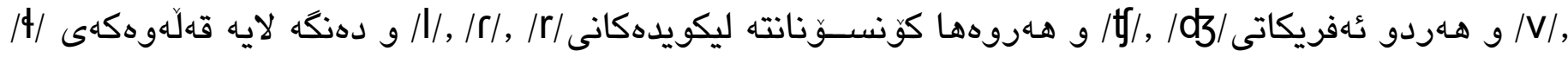

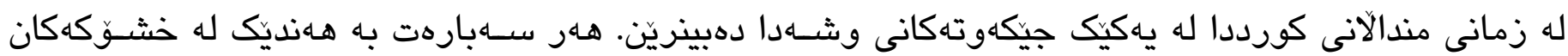

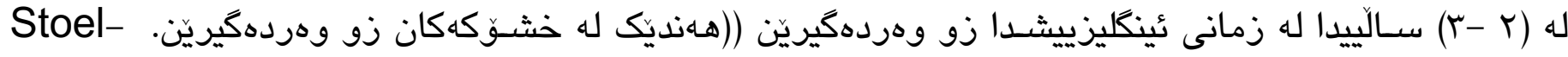

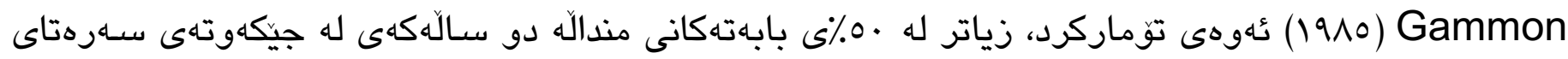

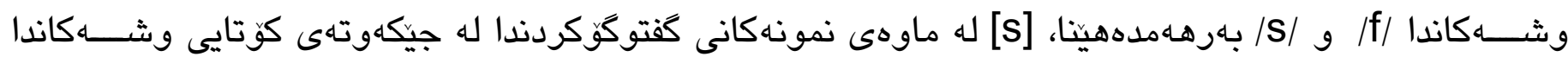

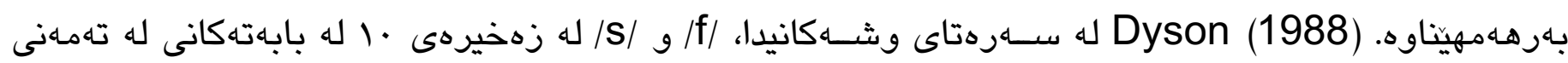

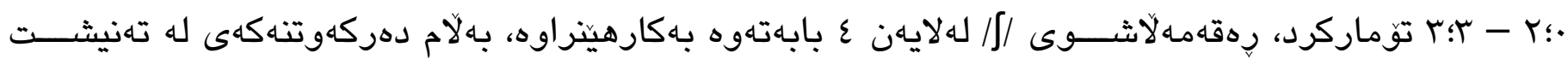

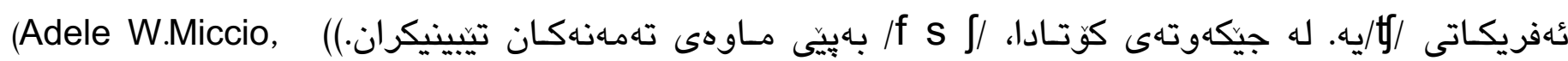
Dennis R. Ingrisano, 2000, 215)

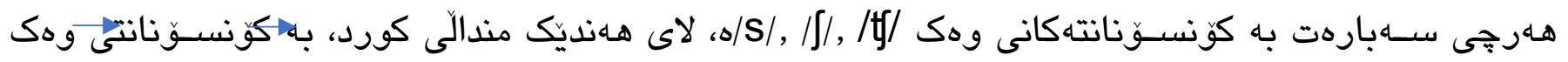

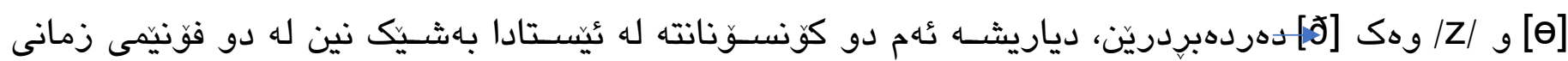

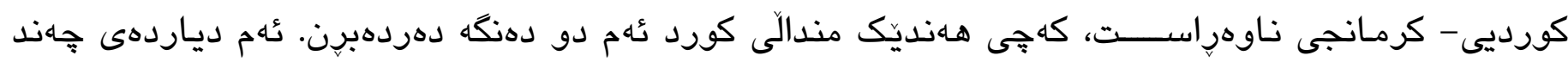

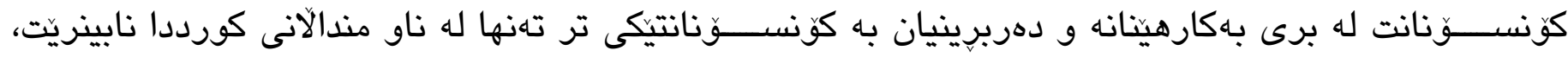

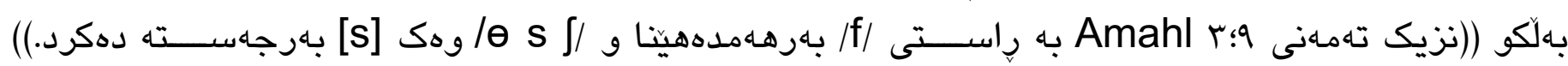

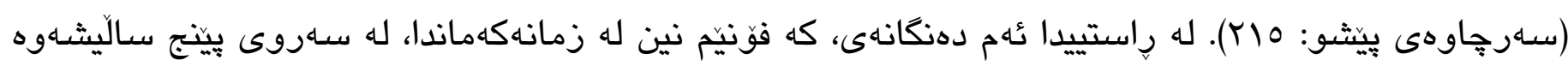

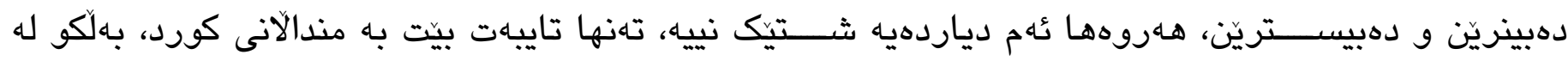

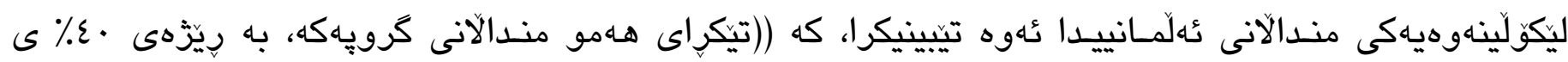

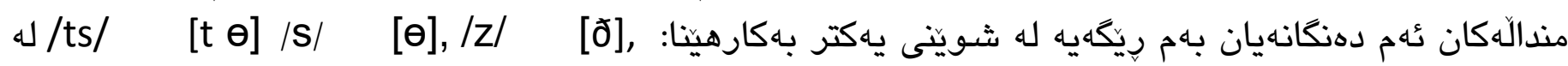

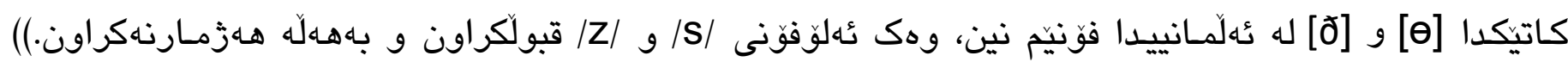
(FOX, 2000, 42)

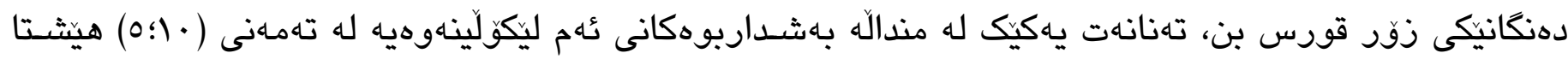

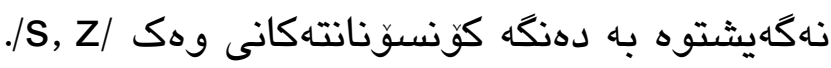

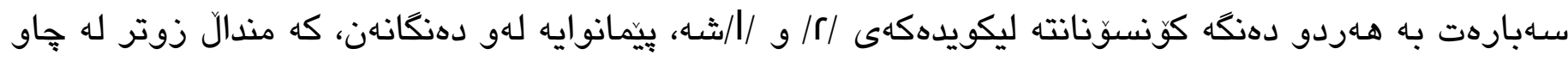

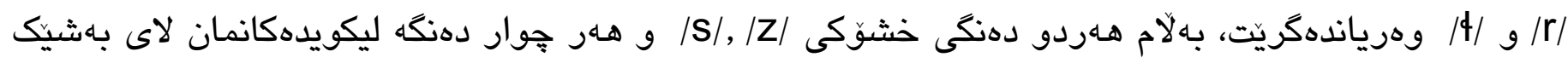

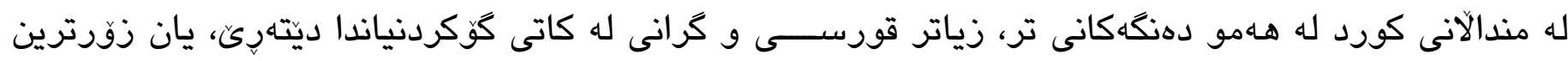

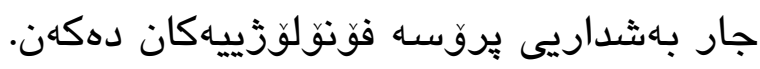

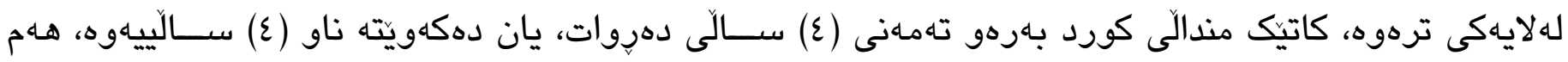

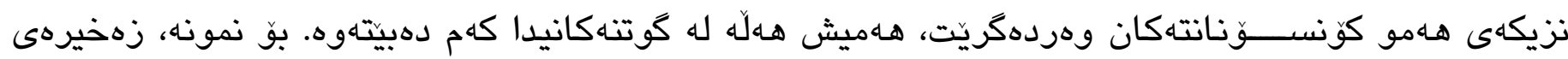

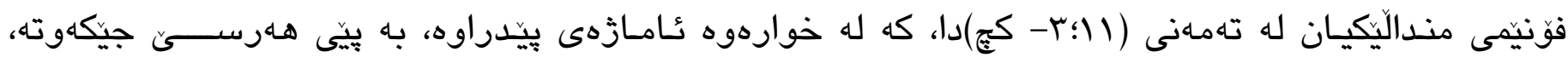

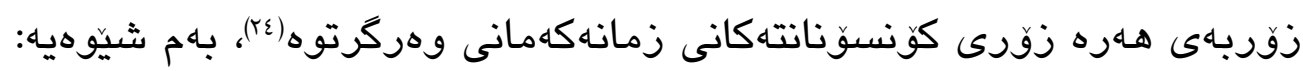

خشتهى زماره (Y (I): زهخيرهى فؤنيمى له تهمهنى (Iا!r-كج) 


\begin{tabular}{|c|c|c|c|c|}
\hline كوّتا Final & ناوهراست Medial & سلهرهتا Initial & 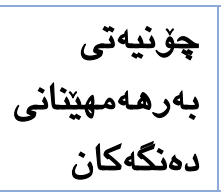 & $\begin{array}{l}r ! 1) \\
\text { (ك) }\end{array}$ \\
\hline$t, d, k, q, g$ & $\begin{array}{l}b, p, t, d, k, g \\
q,\end{array}$ & $\begin{array}{l}b, p, t, d, k, g \\
q, ?\end{array}$ & وهنتاو & \\
\hline \multirow[t]{2}{*}{$m, n, \eta$} & $\mathrm{m}, \mathrm{n}$ & $m, n$ & لوتيى & \\
\hline & $w, j$ & $w, j$ & خزاو & \\
\hline $\begin{array}{l}v, s, z, 3, x, \\
\hbar, \int, \gamma,\end{array}$ & $\begin{array}{l}f, v, s, z, x, \gamma, S, \\
h, \int\end{array}$ & $\begin{array}{l}f, s, z, x, \gamma, \hbar, \\
\varsigma, h, \int\end{array}$ & خشوَك & \\
\hline$t, d 3$ & $t y, d 3$ & $d z, t$ & ئدفريكات & \\
\hline 1,4 & 1,4 & $\mathrm{I}$ & لايى & \\
\hline$r, r$ & $r, r$ & $r$ & للهروَى & \\
\hline
\end{tabular}

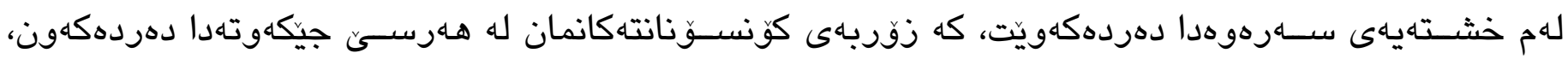

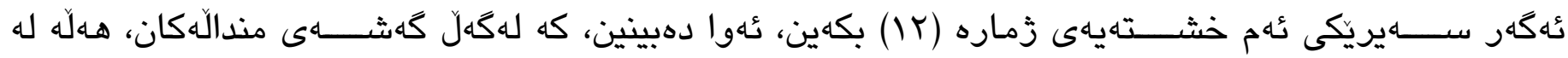

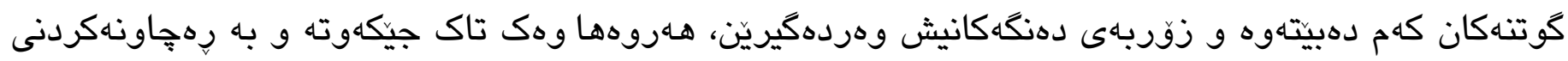

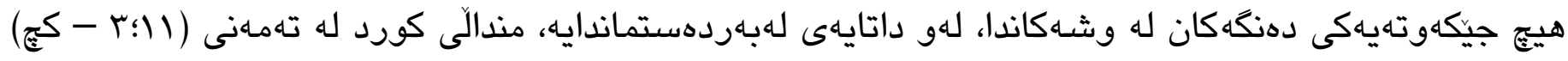

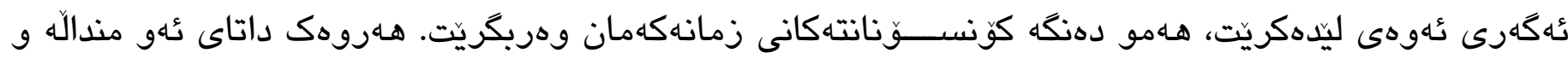

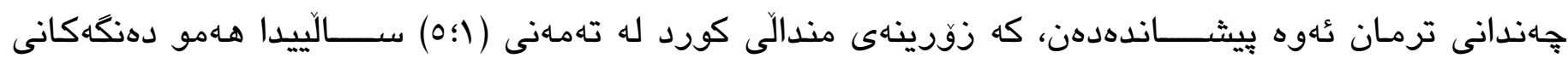

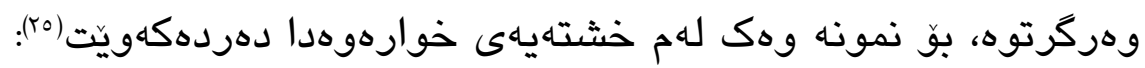




\begin{tabular}{|c|c|c|c|c|c|c|c|c|c|c|c|c|c|c|c|c|c|c|c|c|}
\hline \multicolumn{2}{|c|}{ ד $7: 0$} & \multicolumn{2}{|c|}{$7 !$} & \multicolumn{2}{|c|}{$0 ! V$} & \multicolumn{2}{|c|}{ ك } & \multicolumn{2}{|c|}{$0 ! 1$} & \multicolumn{2}{|c|}{$\varepsilon ! 9$} & \multicolumn{2}{|c|}{$\leqslant ! V$} & \multicolumn{2}{|c|}{ 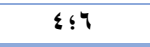 } & \multicolumn{2}{|c|}{$\varepsilon ! r$} & \multicolumn{2}{|c|}{ 11! } & \multirow[b]{2}{*}{$\begin{array}{l}30 د \\
3\end{array}$} \\
\hline$\%$ & زَمار & $\%$ & زَمار & $\%$ & زَمار & $\%$ & زَمار & $\%$ & زَمار & $\%$ & زَمار & $\%$ & زَمار & $\%$ & ز زمار & $\%$ & زَمار & $\%$ & زَمار & \\
\hline 1. & $\wedge$ & 1. & $\wedge$ & 1. & $\wedge$ & 1. & $\wedge$ & 1 & $\wedge$ & 1. & Ir & 1 & $\wedge$ & 1. & $\wedge$ & 1 & $\wedge$ & 1. & $1 \varepsilon$ & b- \\
\hline 1. & $r$ & 1. & $r$ & 1 & $r$ & 1. & $r$ & 1 & $r$ & 1. & $\varepsilon$ & 1. & $r$ & 1. & $r$ & 1 & 1. & 1. & 0 & $\begin{array}{c}-b \\
-\end{array}$ \\
\hline 1 & $r$ & 1. & $r$ & 1. & $r$ & 1. & $r$ & 1 & $r$ & 1. & 1 & 1 & r & 1. & r & 1 & $r$ & & & $-b$ \\
\hline 1. & 1. & 9. & 1. & 9. & 1. & 1. & 1. & 1. & 1. & 1. & V & 1. & 1. & 1. & 1. & 1 & 11 & 1. & $\Lambda$ & $\mathrm{p}-$ \\
\hline 1. & 1 & 1. & 1 & 1. & 1 & 1 & 1 & 1 & 1 & 1. & $r$ & 1 & 1 & 1. & 1 & 1 & 1 & 1 & $r$ & $-p-$ \\
\hline 1. & $r$ & 1. & $r$ & 1. & $r$ & 1 & r & 1. & $r$ & 1. & r & 1. & $r$ & 1. & r & 1 & $r$ & & & $-p$ \\
\hline 1. & $\varepsilon$ & 1. & $\varepsilon$ & 1 & $\varepsilon$ & 1. & $\varepsilon$ & 1 & $\varepsilon$ & 1 & 7 & 1 & $\varepsilon$ & 1. & $\varepsilon$ & 1 & $\wedge$ & 1. & 7 & t- \\
\hline 1. & 0 & 1. & 0 & 1. & 0 & 1. & 0 & 1. & 0 & 1. & $1 \varepsilon$ & 1. & 0 & 1. & 0 & 10 & $1 \varepsilon$ & 1. & 15 & $-t-$ \\
\hline 1. & $\varepsilon$ & 1. & $\varepsilon$ & 1 & $\varepsilon$ & 1 & $\varepsilon$ & 1. & $\varepsilon$ & 1. & r & 1. & $\varepsilon$ & 1. & $\varepsilon$ & 1 & V & 1. & 0 & $-t$ \\
\hline 1. & $\varepsilon$ & 1. & $\varepsilon$ & 1. & $\varepsilon$ & 1. & $\varepsilon$ & 1. & $\varepsilon$ & 1. & 9 & 1. & $\varepsilon$ & vo & $\varepsilon$ & 1 & Ir & 1. & 10 & d- \\
\hline 1. & $r$ & 1. & $r$ & 1. & $r$ & 1 & $r$ & 1 & $r$ & 1. & $\varepsilon$ & 1 & $r$ & 1. & $r$ & 1 & $\varepsilon$ & 1. & V & $-d-$ \\
\hline 1. & 1 & 1. & 1 & 1. & 1 & 1. & 1 & 1. & 1 & 1. & 1 & 1 & 1 & 1. & 1 & . & 1 & 1 & $r$ & $-d$ \\
\hline 1. & 9 & AN & 9 & 1 & 9 & 1 & 9 & 1 & 9 & 1 & 11 & 1 & 9 & $\Lambda \wedge$ & 9 & 1 & IV & 1 & 1. & k- \\
\hline 1. & 7 & 1. & 7 & 1 & 7 & 1 & 7 & 1 & 7 & 1 & V & 1 & 7 & 1. & 7 & 1 & Ir & 1 & Ir & $-k-$ \\
\hline 1. & 7 & 1. & 7 & 1 & 7 & 1 & 7 & 1 & 7 & 1 & 7 & 1 & 7 & 1. & 7 & 1 & $\Lambda$ & 1 & Ir & $-k$ \\
\hline 1. & 0 & 1. & 0 & 1 & 0 & 1 & 0 & 1 & 0 & 1 & V & 1 & 0 & 1 & 0 & 1 & 9 & 1 & V & g- \\
\hline Vo & $\varepsilon$ & 1. & $\varepsilon$ & 1 & $\varepsilon$ & 1 & $\varepsilon$ & 1 & $\varepsilon$ & 1 & $r$ & 1 & $\varepsilon$ & 1 & $\varepsilon$ & 1 & 1. & 1 & $\Lambda$ & $-g-$ \\
\hline 1. & 1 & 1. & 1 & 1 & 1 & 1 & 1 & 1 & 1 & 1 & $r$ & 1 & 1 & 1. & 1 & 1 & $r$ & 1 & r & $-g$ \\
\hline 1. & $r$ & 1. & $r$ & 1 & $r$ & 1 & $r$ & 1 & $r$ & 1 & 0 & 1 & $r$ & 1. & $r$ & 1 & $1 \varepsilon$ & 1 & V & q- \\
\hline 1. & 1 & 1. & 1 & 1 & 1 & 1 & 1 & 1 & 1 & 1 & 1 & 1 & 1 & 1. & 1 & 1 & $\varepsilon$ & 1 & $r$ & $-q-$ \\
\hline 1 & r & 1. & $r$ & 1 & $r$ & 1 & r & 1 & $r$ & 1 & 1 & 1 & $r$ & 1 & r & 1 & $r$ & 1 & r & $-q$ \\
\hline 1. & $r$ & 1. & $r$ & 1 & $r$ & 1. & $r$ & 1. & $r$ & 1. & $r$ & 1 & $r$ & 1. & $r$ & 1 & 11 & 1 & $\varepsilon$ & ?- \\
\hline 1 & 1. & 1. & 1. & 1 & 1. & 1 & 1. & 1 & 1. & 1 & 17 & 1 & 1. & 1 & 1. & 1 & $1 \varepsilon$ & 1 & IV & m- \\
\hline 1. & $\varepsilon$ & 1. & $\varepsilon$ & 1 & $\varepsilon$ & 1 & $\varepsilon$ & Vo & $\varepsilon$ & 1 & 7 & 1 & $\varepsilon$ & 1. & $\varepsilon$ & 10 & $V$ & 1 & 11 & $-m$ \\
\hline 1. & 1 & 1. & 1 & 1 & 1 & 1 & 1 & 1 & 1 & 1 & $\varepsilon$ & 1 & 1 & 1 & 1 & 1 & 1. & 1 & 1. & $-m$ \\
\hline 1 & $r$ & 1. & $r$ & 1 & $r$ & 1 & $r$ & 1 & $r$ & 1 & 1 & 1 & $r$ & 1 & $r$ & 1 & $\varepsilon$ & 1 & $\varepsilon$ & $n-$ \\
\hline 1 & 7 & 1. & 7 & 1 & 7 & 1 & 7 & 1 & 7 & 1 & זו & 1 & 7 & 1 & 7 & 1 & rT & 1 & 17 & $-n-$ \\
\hline 1. & $r$ & 1. & $r$ & 1 & $r$ & 1 & $r$ & 1 & $r$ & 1 & 0 & 1 & $r$ & 1. & $r$ & 1 & Ir & 1 & $V$ & $-n$ \\
\hline 1 & 1 & 1. & 1 & 1 & 1 & 1 & 1 & 1 & 1 & 1 & 1 & 1 & 1 & 1 & 1 & 1 & r & 1 & 1 & $-\eta$ \\
\hline 1. & 1 & 1. & 1 & 1 & 1 & 1 & 1 & 1 & 1 & 0. & $r$ & 1 & 1 & 1. & 1 & 77 & 7 & 1 & $r$ & W- \\
\hline 1 & $r$ & 1. & r & 1 & $r$ & 1 & $r$ & 1 & $r$ & 1 & 11 & 77 & $r$ & 1 & $r$ & 1 & 9 & 1 & 1. & $-w-$ \\
\hline o. & r & 1. & $r$ & 0. & $r$ & 1 & $r$ & 0. & $r$ & 1 & 1 & 0. & $r$ & 0. & $r$ & 77 & $r$ & 1 & $r$ & j- \\
\hline
\end{tabular}




\begin{tabular}{|c|c|c|c|c|c|c|c|c|c|c|c|c|c|c|c|c|c|c|c|c|}
\hline . & 1 & . & 1 & 1. & 1 & 1. & 1 & 1. & 1 & 1. & V & 1. & 1 & . & 1 & 1. & V & 1. & $V$ & $-j-$ \\
\hline 1. & $r$ & 1. & T & 1. & $r$ & 0. & r & 1. & r & 0 . & r & 1. & r & 1. & T & 7. & 0 & 1. & $r$ & $f-$ \\
\hline 1. & $r$ & 1. & $r$ & 1. & r & 1. & r & 1. & r & 1. & 1 & 1. & r & 1. & $r$ & 1. & 7 & 1. & $\varepsilon$ & $-f-$ \\
\hline 1. & 1 & 1. & 1 & 1. & 1 & 1. & 1 & 1. & 1 & & & 1. & 1 & 1. & 1 & & & & & $-f$ \\
\hline 1. & 1 & 1. & 1 & 1. & 1 & 1. & 1 & 1. & 1 & & & 1. & 1 & 1. & 1 & & & & & V- \\
\hline 1. & 1 & 1. & 1 & 1. & 1 & 1. & 1 & 1. & 1 & & & 1. & 1 & 1. & 1 & 1. & 1 & 1. & 1 & $-V-$ \\
\hline 1. & r & 1. & T & 1. & $r$ & 1. & T & 1. & r & & & 1. & r & 1. & $r$ & & & 1. & 1 & $-v$ \\
\hline 1. & r & 1. & r & . & r & 1. & r & 1. & r & 10 & V & 0. & r & 1. & r & . & V & 1. & $\wedge$ & S- \\
\hline 1. & $\wedge$ & 1. & $\wedge$ & . & $\wedge$ & 1. & $\wedge$ & AV & $\wedge$ & 1. & $\varepsilon$ & 1. & $\wedge$ & vo & $\wedge$ & . & IT & 1. & $1 \varepsilon$ & -s- \\
\hline 1. & 1 & 1. & 1 & . & 1 & 1. & 1 & 1. & 1 & 1. & r & 1. & 1 & 1. & 1 & . & $\varepsilon$ & 1. & 1 & -s \\
\hline 1. & r & 1. & r & . & $r$ & 1. & Y & 1. & Y & 1. & r & 1. & r & 1. & r & . & 7 & 1. & 0 & Z- \\
\hline 1. & r & 1. & $r$ & . & r & 1. & T & 1. & Y & Vo & $\varepsilon$ & 1. & $r$ & 1. & $r$ & . & V & 1. & 9 & $-z-$ \\
\hline 1. & $r$ & 1. & $r$ & . & $r$ & 1. & $r$ & 1. & $r$ & 1. & $\varepsilon$ & 77 & $r$ & 77 & $r$ & . & r & 1. & 0 & $-z$ \\
\hline 1. & 0 & 1. & 0 & 1. & 0 & 1. & 0 & 1. & 0 & 1. & V & $\varepsilon \cdot$ & 0 & A. & 0 & 1. & V & 1. & $\wedge$ & $\int-$ \\
\hline 1. & $V$ & 1. & V & 1. & V & 1. & V & 1. & V & 1. & $\wedge$ & $\leqslant Y$ & V & 10 & V & 1. & 1. & 1. & $\varepsilon$ & $-\int-$ \\
\hline 1. & 1 & 1. & 1 & 1. & 1 & 1. & 1 & 1. & 1 & 1. & $\varepsilon$ & 1. & 1 & 1. & 1 & 0. & $r$ & 1. & $r$ & $-\int$ \\
\hline 1. & $r$ & 1. & r & i. & r & 1. & r & 1. & r & 1. & 1 & 0. & r & . & $r$ & 1. & 1 & & & 3- \\
\hline 1. & 1 & 1. & 1 & 1. & 1 & 1. & 1 & 1. & 1 & 1. & 1 & . & 1 & . & 1 & 1. & $\varepsilon$ & & & $-3-$ \\
\hline 1. & 1 & 1. & 1 & 1. & 1 & 1. & 1 & 1. & 1 & 1. & r & . & 1 & 1. & 1 & 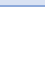 & & 1. & $r$ & -3 \\
\hline vo & $\varepsilon$ & 1. & $\varepsilon$ & 1. & $\varepsilon$ & 1. & $\varepsilon$ & 1. & $\varepsilon$ & 1. & r & 1. & $\varepsilon$ & 1. & $\varepsilon$ & 1. & $\varepsilon$ & 1. & 7 & $x-$ \\
\hline 1. & 1 & 1. & 1 & 1. & 1 & 1. & 1 & 1. & 1 & 1. & $r$ & 1. & 1 & 1. & 1 & 1. & 0 & 1. & $r$ & $-x-$ \\
\hline 1. & 1 & 1. & 1 & 1. & 1 & 1. & 1 & 1. & 1 & 1. & $r$ & 1. & 1 & 1. & 1 & 1. & T & 1. & $r$ & $-x$ \\
\hline o. & r & 1. & T & 0. & $r$ & 1. & T & 0. & r & & & 1. & Y & 1. & $r$ & & & 0. & 1 & $\gamma-$ \\
\hline 1. & 1 & 1. & 1 & 1. & 1 & 1. & 1 & 1. & 1 & . & 1 & 1. & 1 & 1. & 1 & 1. & 1 & 1. & r & $-\gamma-$ \\
\hline 1. & 1 & 1. & 1 & 1. & 1 & 1. & 1 & 1. & 1 & & & 1. & 1 & 1. & 1 & & & 1. & 1 & $-\gamma$ \\
\hline 1. & 1 & i. & 1 & 1. & 1 & 1. & 1 & 1. & 1 & 1. & r & 1. & 1 & 1. & 1 & 1. & $r$ & 1. & 1 & $\hbar-$ \\
\hline 1. & 1 & 1. & 1 & . & 1 & 1. & 1 & 1. & 1 & 1. & 1 & 1. & 1 & 1. & 1 & . & 1 & & & $-\hbar-$ \\
\hline 1. & $r$ & 1. & $r$ & 1. & $r$ & 1. & r & 1. & r & 1. & 1 & 1. & r & 1. & r & 1. & $r$ & 1. & $r$ & $-\hbar$ \\
\hline 1. & 1 & 1. & 1 & 1. & 1 & 1. & 1 & 1. & 1 & 1. & 1 & 1. & 1 & 1. & 1 & . & 1 & 1. & $r$ & S- \\
\hline 1. & 1 & 1. & 1 & 1. & 1 & 1. & 1 & 1. & 1 & 1. & 1 & 1. & 1 & 1. & 1 & 0. & $r$ & 1. & 1 & $-\zeta-$ \\
\hline . & 1 & . & 1 & 1. & 1 & . & 1 & 1. & 1 & & & 1. & 1 & 1. & 1 & & & & & $-\zeta$ \\
\hline 1. & r & 1. & T & 0. & r & 1. & r & 1. & r & A. & 0 & 1. & r & 1. & r & Vo & $\varepsilon$ & NT & 7 & h- \\
\hline 1. & 1 & 1. & 1 & 1. & 1 & 1. & 1 & 1. & 1 & 1. & r & 1. & 1 & 1. & 1 & 1. & $r$ & 1. & $r$ & -h- \\
\hline
\end{tabular}




\begin{tabular}{|c|c|c|c|c|c|c|c|c|c|c|c|c|c|c|c|c|c|c|c|c|}
\hline 1. & $r$ & 1. & r & 1. & $r$ & 1 & $r$ & 1. & r & 1. & $r$ & • & r & 1. & $r$ & 1 & 1 & 1. & 1 & ds- \\
\hline 1. & 1 & 1 & 1 & 1. & 1 & 1 & 1 & 1 & 1 & 1 & $r$ & . & 1 & 1 & 1 & 1 & 7 & 1. & $r$ & $-d z-$ \\
\hline 1. & 1 & 1 & 1 & 1. & 1 & 1. & 1 & 1. & 1 & 1. & 1 & 1. & 1 & 1. & 1 & 1. & 1 & 1. & 1 & $-d 3$ \\
\hline 1. & 1 & 1. & 1 & 1. & 1 & 1 & 1 & 1. & 1 & 1. & $r$ & 1. & 1 & . & 1 & 1 & $\Lambda$ & 1. & V & tf- \\
\hline 1. & 1 & 1. & 1 & 1. & 1 & 1 & 1 & 1 & 1 & & & . & 1 & 1. & 1 & 1. & $\varepsilon$ & vo & $\varepsilon$ & $-t 5$ \\
\hline 1. & $r$ & 1. & $r$ & 1. & $r$ & 1 & $r$ & 1 & $r$ & 1 & r & 1. & $r$ & 1 & $r$ & 1. & 1 & 1. & $r$ & $-t 5$ \\
\hline 1. & $r$ & 77 & $r$ & 1. & $r$ & 1 & $r$ & 1 & $r$ & $\varepsilon$ & 0 & 1 & $r$ & 1 & $r$ & 1. & 7 & 1. & $\varepsilon$ & I- \\
\hline 1. & $\Lambda$ & 1. & $\Lambda$ & 1. & $\Lambda$ & AV & $\wedge$ & 1. & $\Lambda$ & 1. & 1. & 1. & $\Lambda$ & Tr & $\wedge$ & $\wedge 1$ & 11 & 1. & $\Lambda$ & $-1-$ \\
\hline 1. & $r$ & 1 & r & 1. & $r$ & 1. & $r$ & 1 & $r$ & 1. & $r$ & 1 & r & 0. & $r$ & 1. & $r$ & 1. & $r$ & -1 \\
\hline$\Lambda V$ & $\Lambda$ & 1. & $\Lambda$ & 1. & $\Lambda$ & 1. & $\wedge$ & 1 & $\Lambda$ & 1 & $\Lambda$ & . & $\Lambda$ & Tr & $\Lambda$ & NT & IT & 1. & 10 & $-4-$ \\
\hline 1. & $r$ & 1. & r & 1. & $r$ & 1 & $r$ & 1. & r & 1. & $r$ & . & $r$ & 1. & $r$ & 1. & $V$ & 1. & $r$ & -4 \\
\hline 1. & $1 \leq$ & 1. & $1 \leqslant$ & 1. & $1 \leq$ & $9 r$ & $1 \varepsilon$ & 1 & $1 \varepsilon$ & $V V$ & 11 & . & $1 \varepsilon$ & 1 & $1 \leqslant$ & 10 & rA & 1. & ro & $-r-$ \\
\hline 1. & 9 & 1. & 9 & 1. & 9 & 1. & 9 & 1 & 9 & qY & $1 \pi$ & . & 9 & 1. & 9 & 1. & 17 & 1. & 9 & $-r$ \\
\hline o. & $r$ & 1. & $r$ & 1. & $r$ & 1. & r & 1. & $r$ & 1. & $\varepsilon$ & . & $r$ & 1. & r & 1. & 1 & 1. & 0 & $r-$ \\
\hline 77 & $r$ & 1 & $r$ & 1. & $r$ & 1 & $r$ & 1 & $r$ & 1 & 0 & . & $r$ & 1 & $r$ & $q r$ & IT & 1. & $\varepsilon$ & $-r-$ \\
\hline 1. & 1 & 1. & 1 & 1. & 1 & 1. & 1 & 1. & 1 & 1. & 1 & . & 1 & 1. & 1 & 1 & $r$ & 1. & $r$ & $-r$ \\
\hline
\end{tabular}

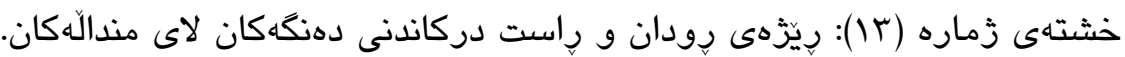

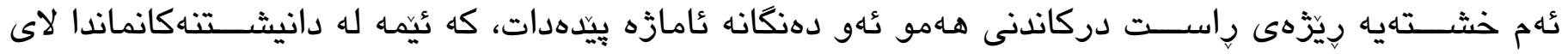

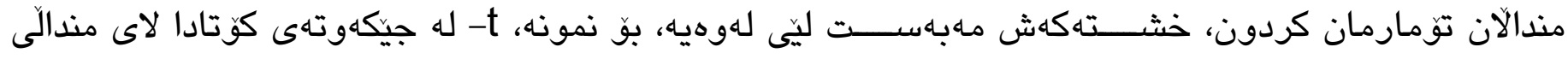

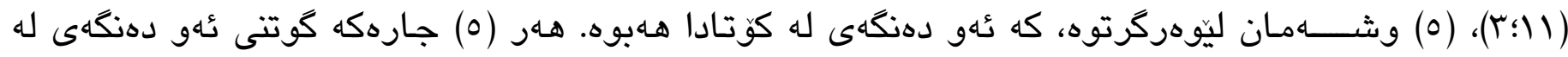

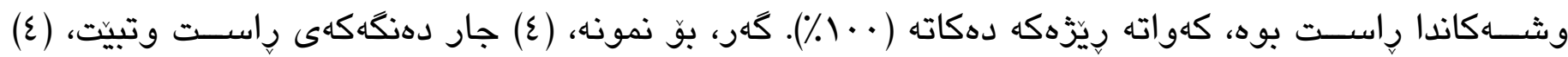

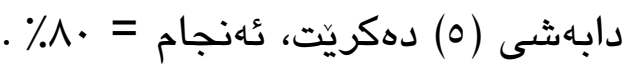

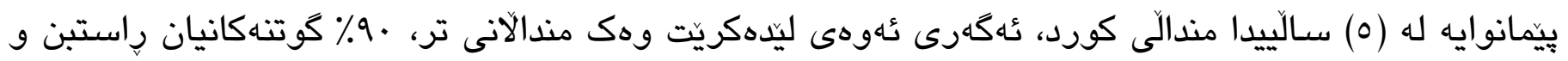

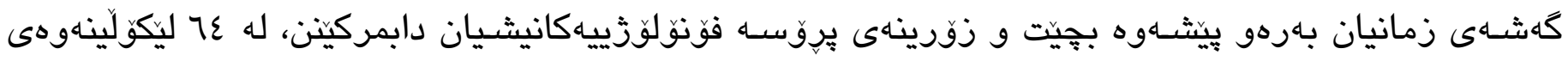

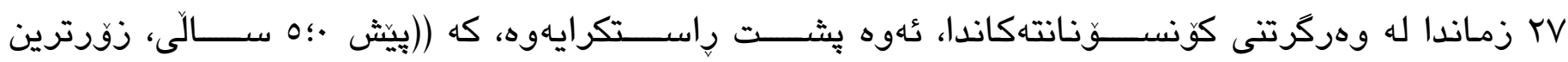

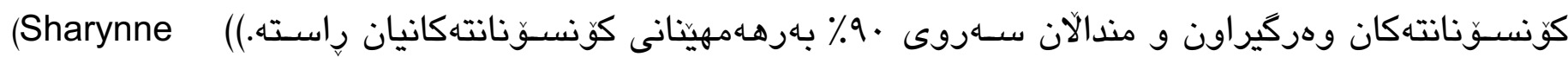
Mcleod and Kathryn Crowe, 2018, 1563)

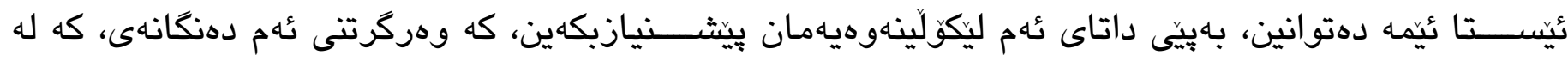

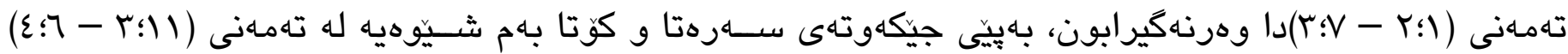
دهنكَكان وهردهكيرين: 


\begin{tabular}{|c|c|}
\hline كونسوَنانتهـان & تهمهن \\
\hline g & r!וl \\
\hline$q$ & r!ו \\
\hline$\eta$ & r!ו \\
\hline$\gamma$ & |ו \\
\hline$\hbar$ & r!ו \\
\hline G & $\varepsilon: 1$ \\
\hline$\S d 3$ & ए!I \\
\hline$\S \downarrow$ & r!I \\
\hline$\S r$ & r!וा \\
\hline
\end{tabular}

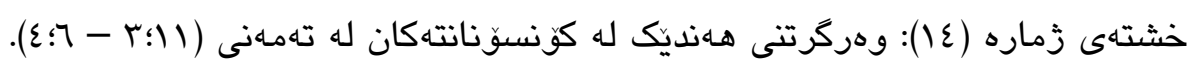

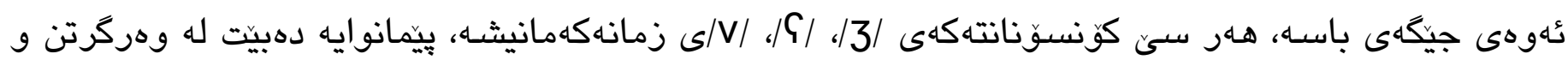

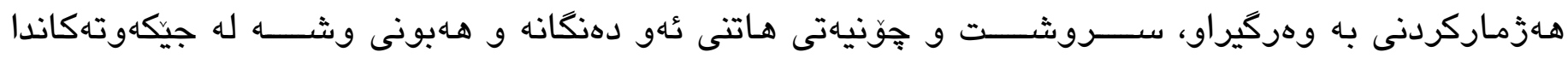

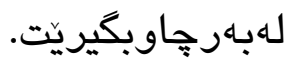

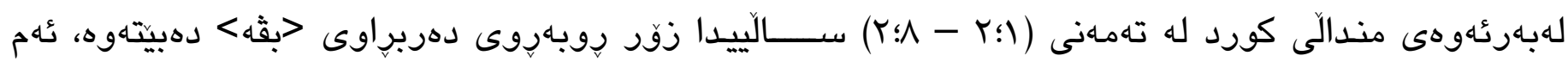

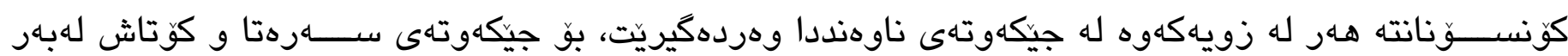

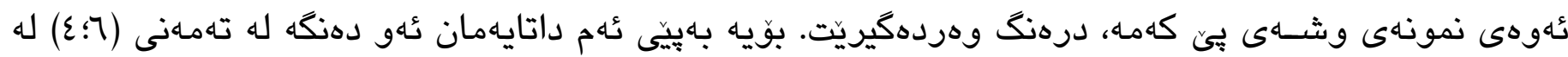

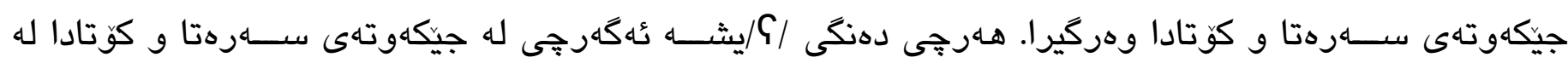

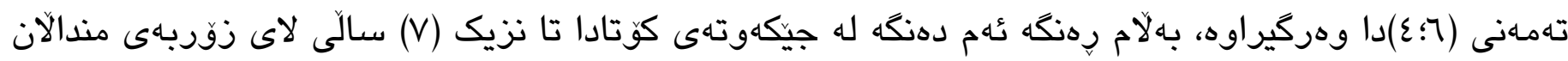

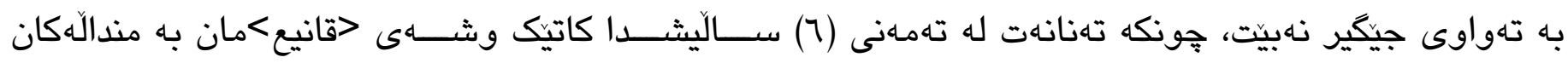

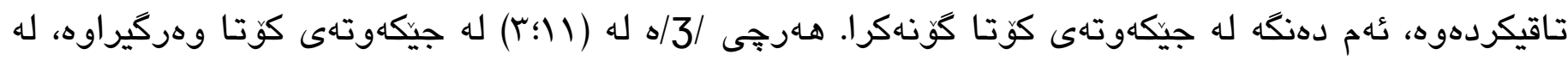

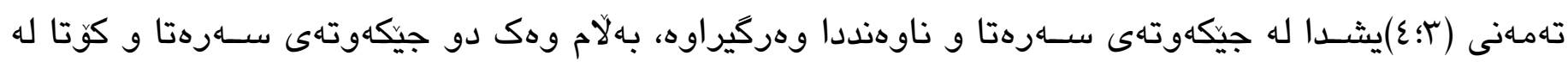

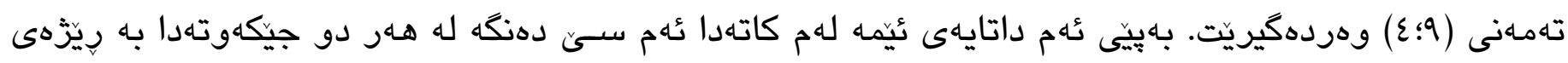

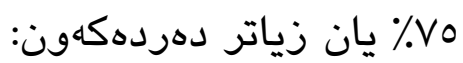

\begin{tabular}{|c|c|}
\hline كونسوَنانتهكان & تهمهن \\
\hline $\mathrm{V}$ & $\varepsilon: 7$ \\
\hline 3 & $\varepsilon: 9$ \\
\hline$\zeta$ & ד! \\
\hline
\end{tabular}

\section{خشتهى زماره (10): وهركرتنى هـنديكِ دهنكى خشوَك.}

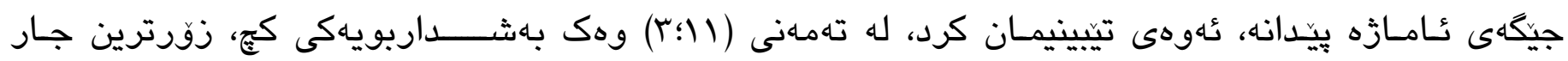

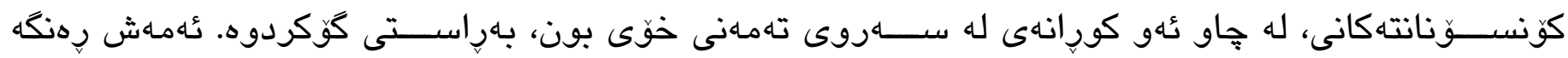

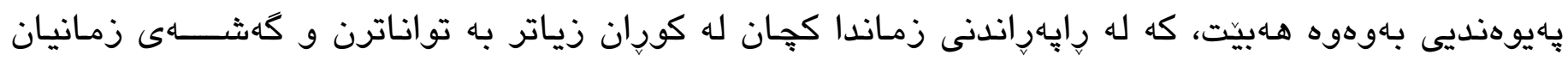

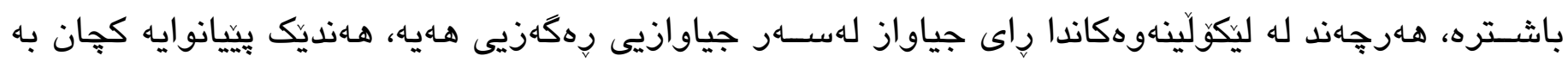

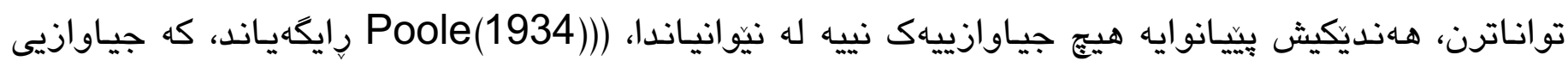




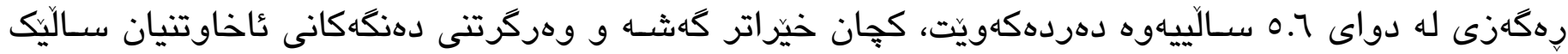

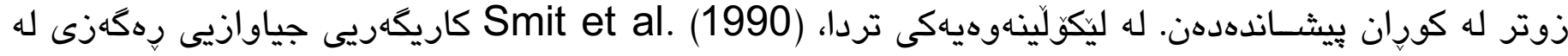

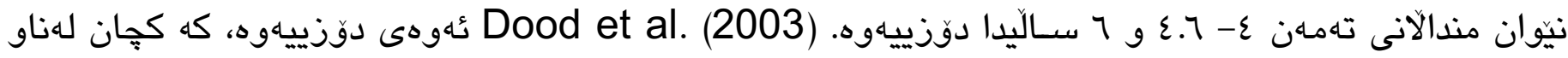

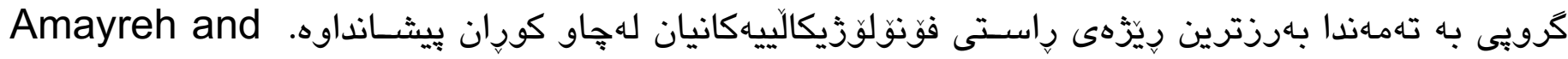

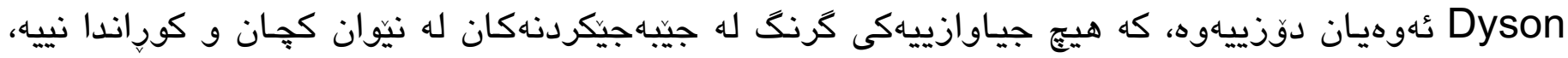

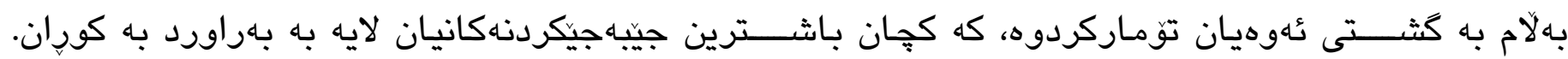
Zarifian et al. (2014)

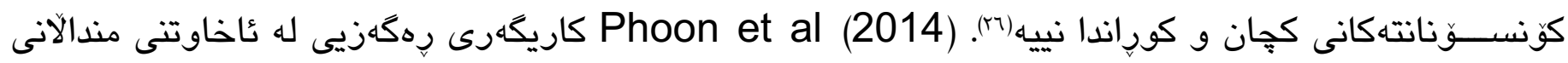

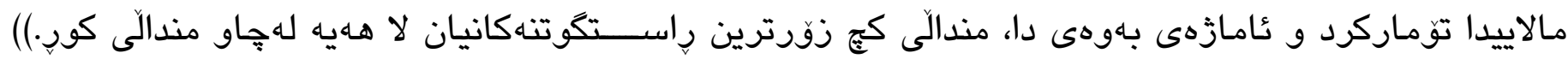
(Shahla Fatemi Syadar, Talieh Zarifian, Yahya Modarresi, Muhamad Sediq Zahedi, Mona

Ebrahimipur, Akbar Biglarian: 2018, 160)

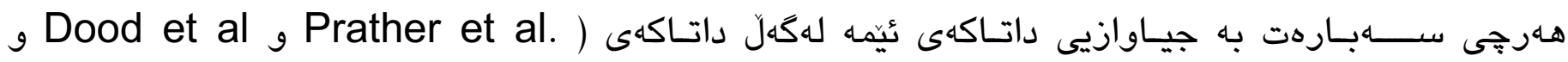
(Amayreh and Dyson

\begin{tabular}{|c|c|c|c|c|c|}
\hline كورد مندالى & $\begin{array}{c}\text { Prather } \\
\text { et al. }\end{array}$ & كان & كورد مندالى & $\begin{array}{c}\text { Prather } \\
\text { et al. }\end{array}$ & نك ده \\
\hline r!II & $r$ & g & $r ! 1$ & r & $\mathrm{m}$ \\
\hline$\S Y \subseteq \varepsilon$ & $\S$ & $S$ & Y & $r$ & $\mathrm{n}$ \\
\hline$\S$ & ع & $r$ & r!I & $r$ & h \\
\hline$r: \mathrm{V}$ & & 1 & 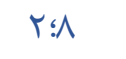 & $r$ & $p$ \\
\hline$\oint r ! 1$ & ऍ؛ & $\int$ & 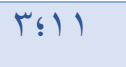 & r & $\eta$ \\
\hline § & ऍ & t5 & $\Gamma ! 1$ & $r \leqq \varepsilon$ & $f$ \\
\hline$\varepsilon \subseteq 9$ & $\varepsilon$ & 3 & $Y \leqq \wedge$ & $r \leqq \varepsilon$ & j \\
\hline 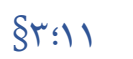 & ${ }^{\circ}+\varepsilon$ & d3 & 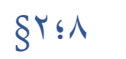 & $r \leqq \varepsilon$ & k \\
\hline$\varepsilon ! 7$ & ${ }^{\circ}+\varepsilon$ & V & r!) & $r ! \varepsilon$ & d \\
\hline$r \leqslant \varepsilon$ & ${ }^{\circ}+\varepsilon$ & z & Y!I & r؛A & w \\
\hline & & & $r \leqq \Lambda$ & $r: \Lambda$ & b \\
\hline & & & $r ! 1$ & $r \leqq \Lambda$ & $t$ \\
\hline
\end{tabular}

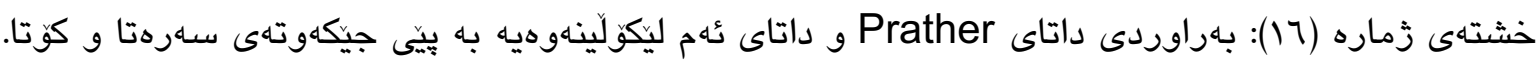

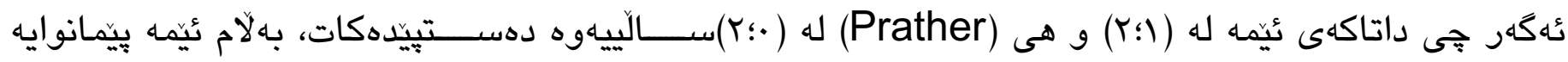

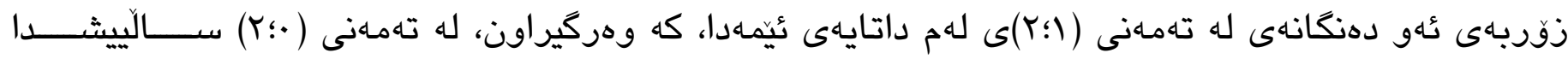

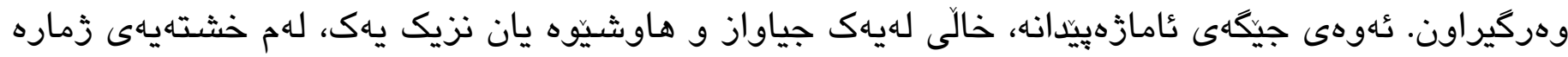

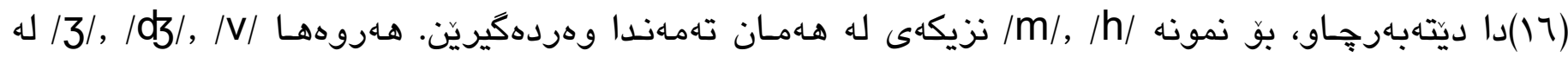

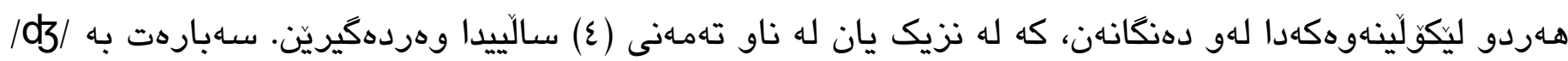

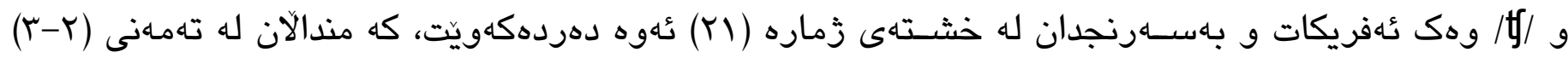




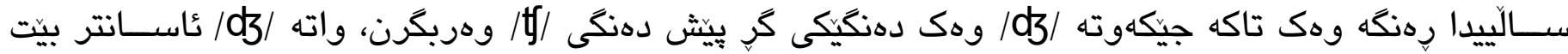

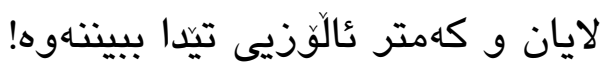

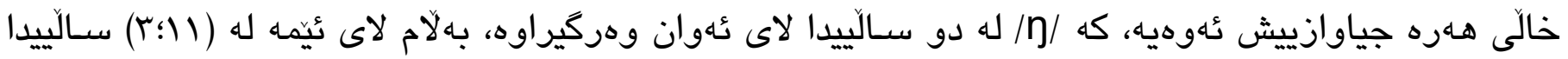

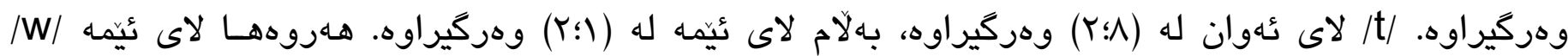

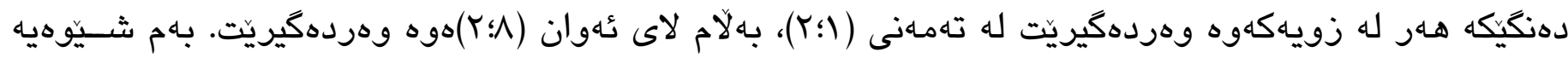

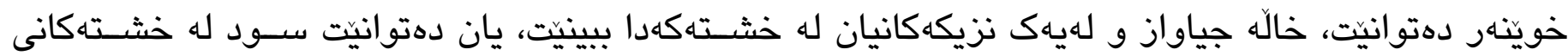

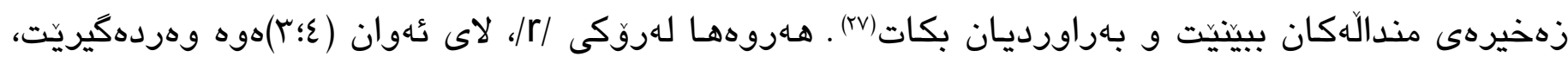

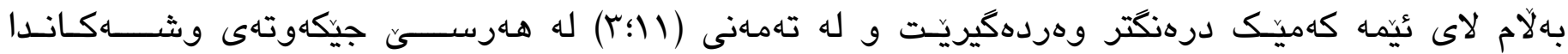
بهاردهوهينتريّت.

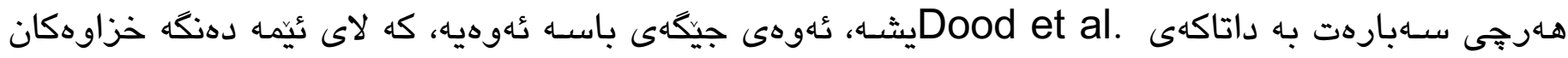

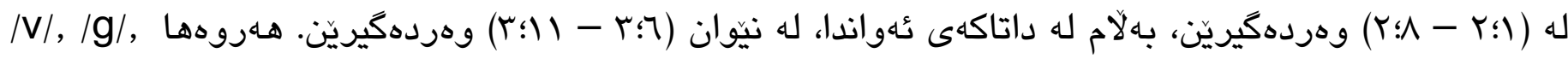

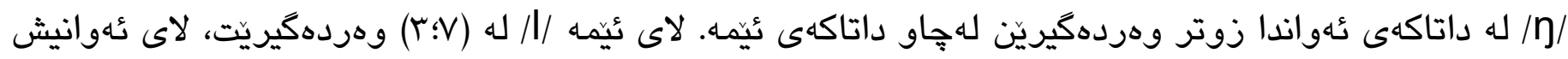

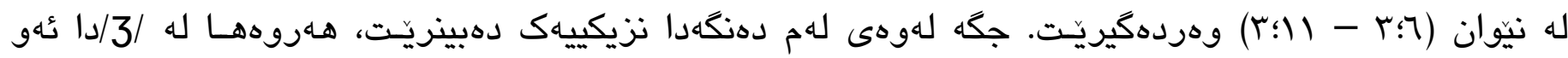

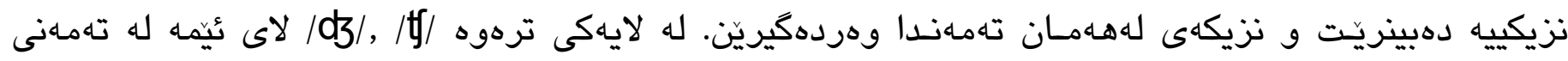

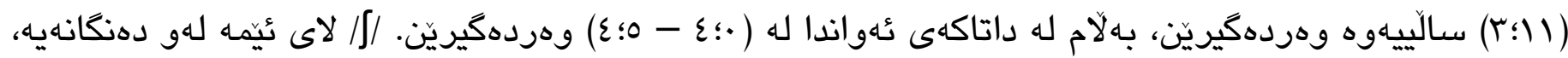

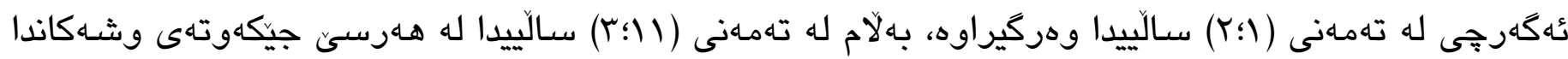

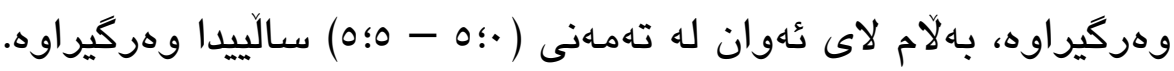

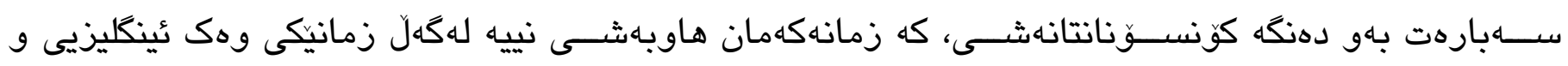

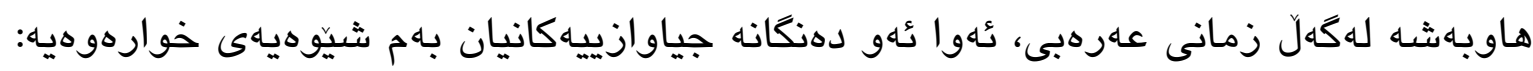

\begin{tabular}{|c|c|c|}
\hline مندالى كورد & $\begin{array}{r}\text { Amayreh and } \\
\text { Dyson }\end{array}$ & دهنگ \\
\hline$r ! I$ & درهنگ/ دواى ع؛7 & $?$ \\
\hline r!ा & ع درهنگ/ دواى & $q$ \\
\hline$\S \varepsilon ! 7$ & درهنگ/ دواى ع؛7 & 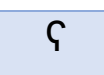 \\
\hline ए!II & ناوهند/ •؛ع - ع؛7 & (b) $y$ \\
\hline$r ! \varepsilon$ & ناوهند / •؛- ع؛7 & $x(x)$ \\
\hline
\end{tabular}

خشتهى زماره (IV): بهراوردى وهركرتنى دهنكه هاوبهشهكانى كوردى و عهرهبى.

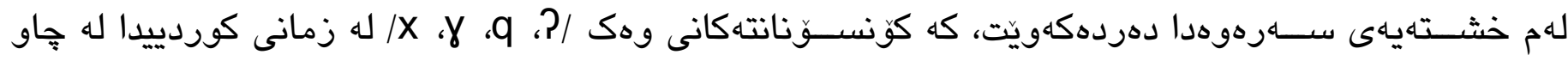

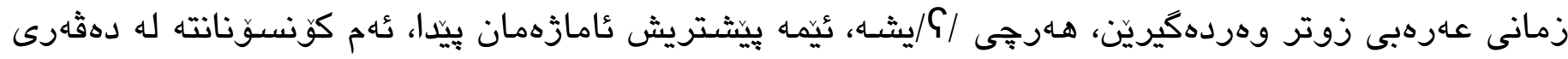

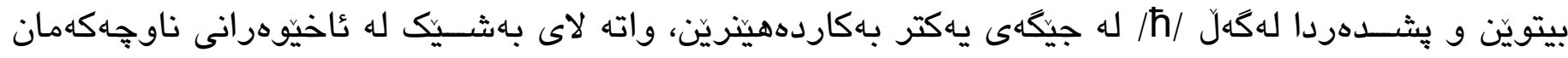

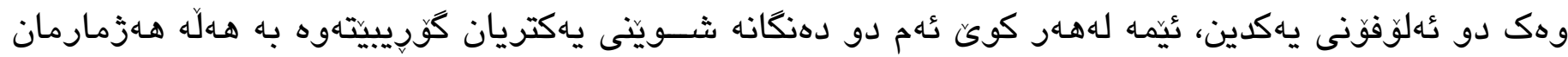

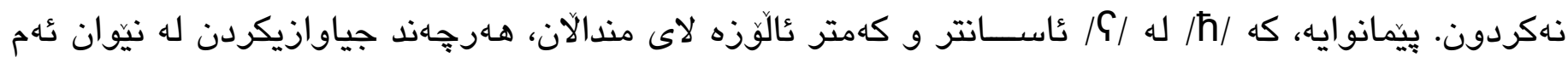

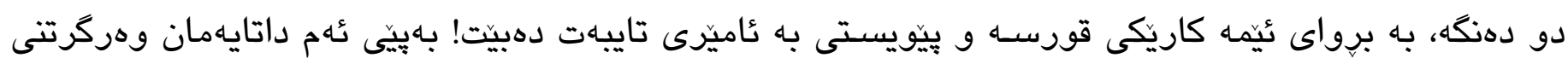




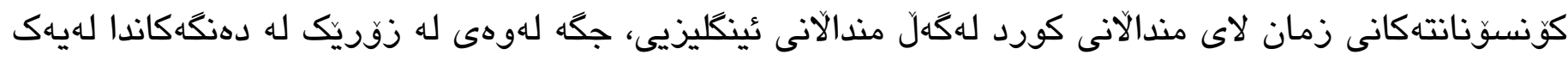

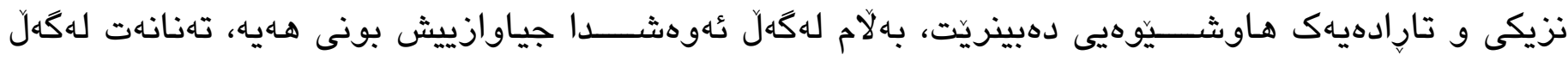

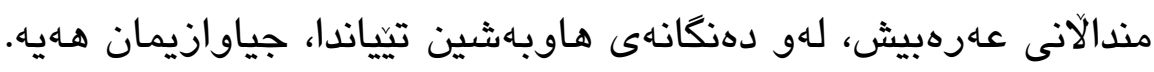

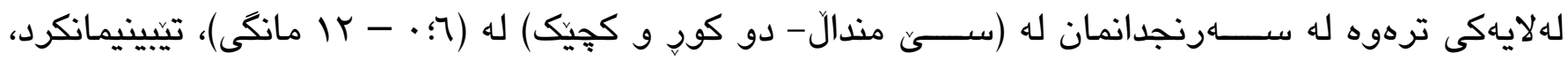

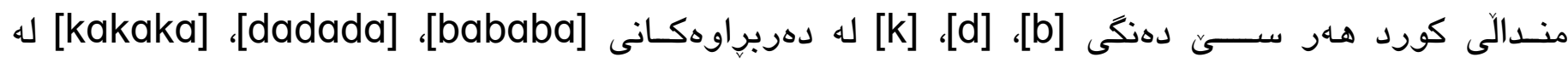

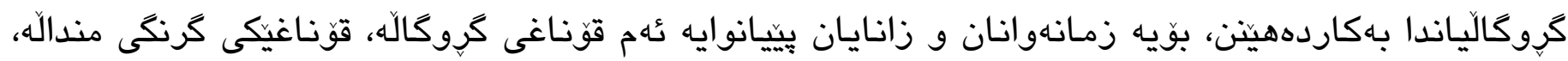

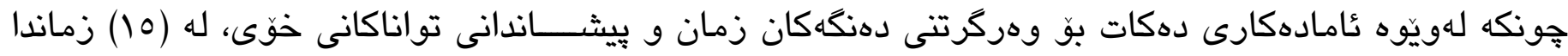

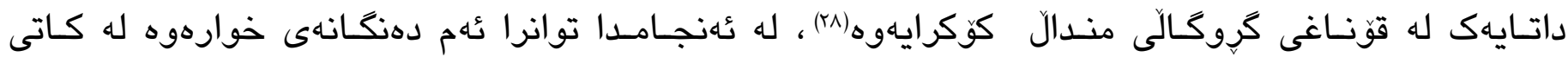

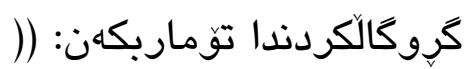

\begin{tabular}{|c|c|}
\hline $\begin{array}{r}\text { كونســونانته دوزراوه جاروباره بـكارهاتوهكان } \\
\text { Infrequently found consonants }\end{array}$ & 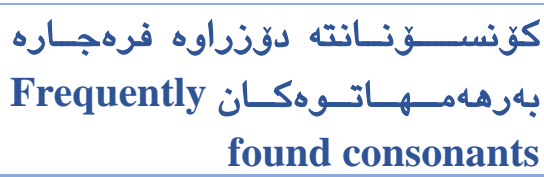 \\
\hline$f, v, \ominus, \succsim$ & $p, b, m$ \\
\hline $\int, 3, t, d z$ & $\mathrm{t}, \mathrm{d}, \mathrm{n}$ \\
\hline \multirow[t]{2}{*}{$I, r, \eta$} & $\mathrm{k}, \mathrm{g}$ \\
\hline & $s, h, w, j$ \\
\hline
\end{tabular}

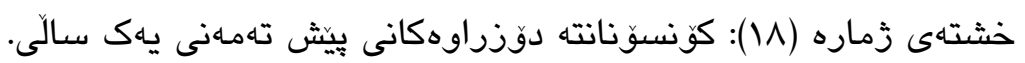

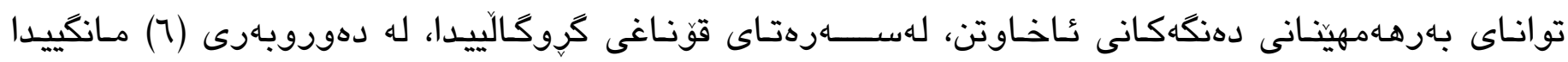

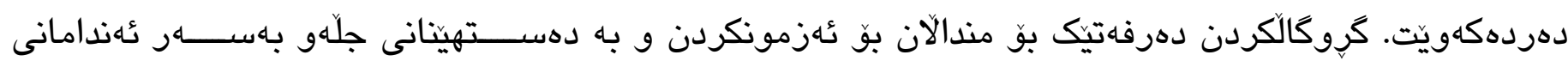

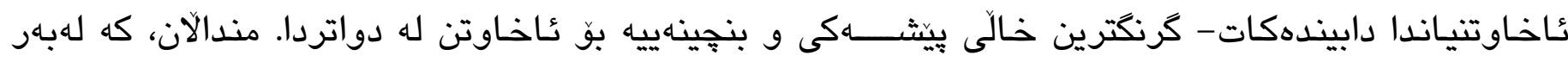

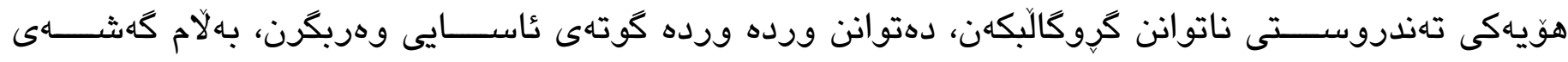

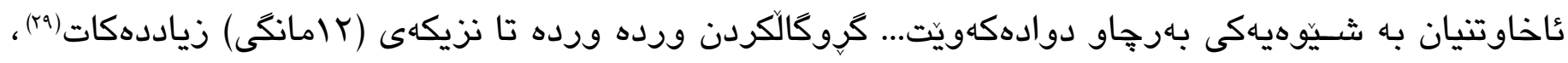

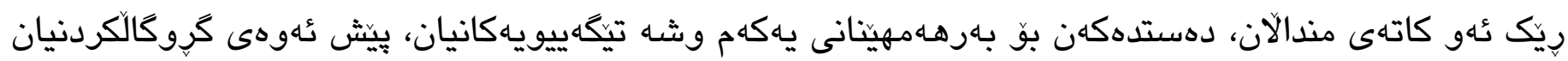

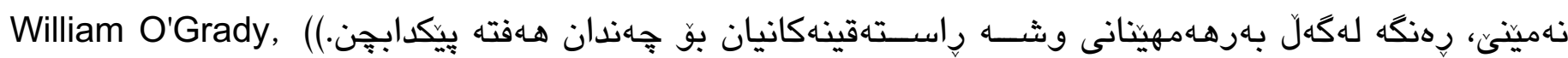
(Sook Whan Cho, 2005, 365)

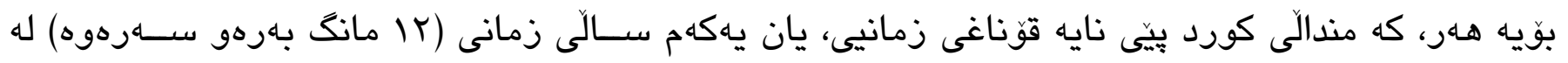

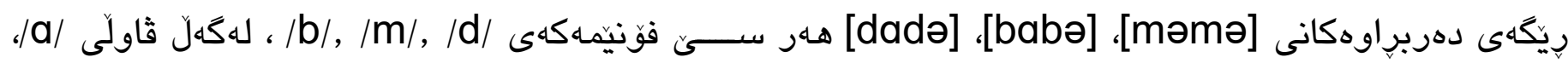

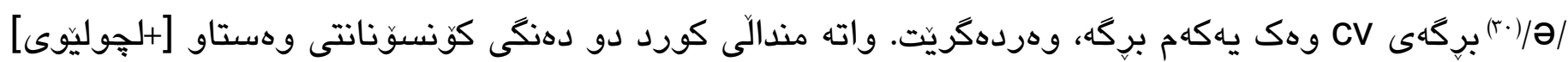

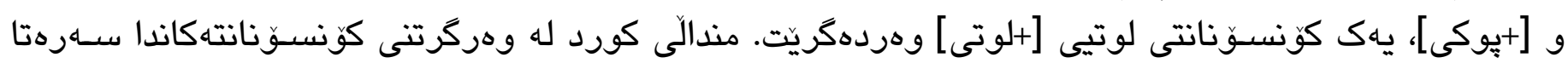

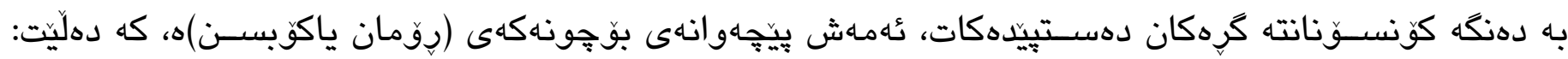

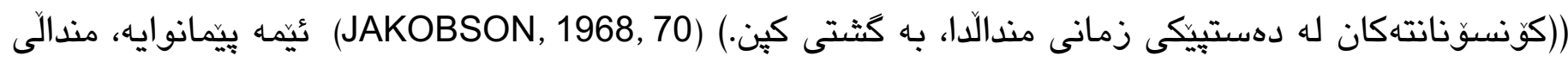

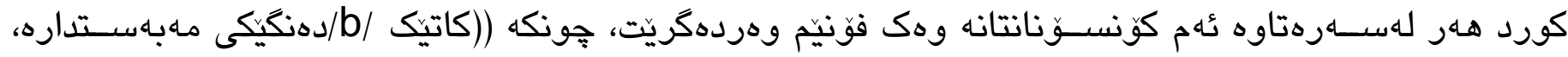

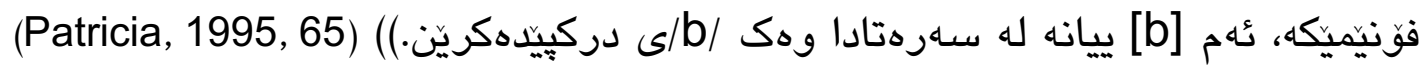

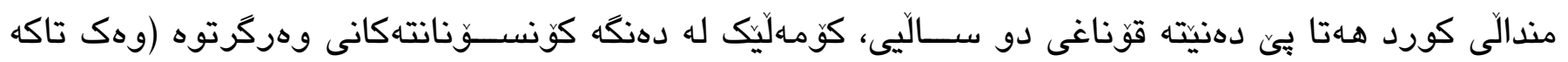

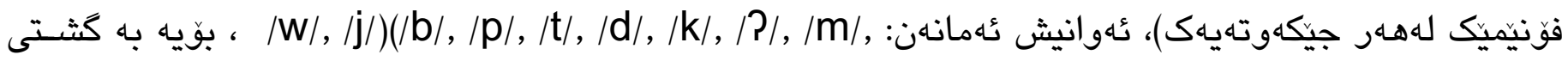




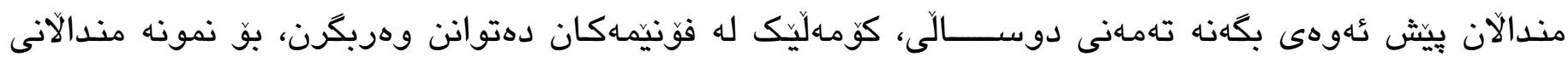

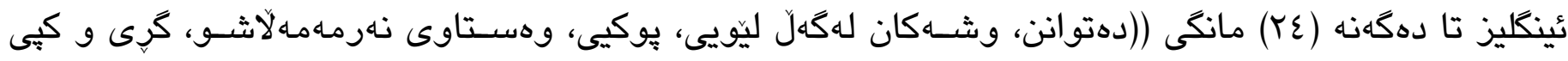

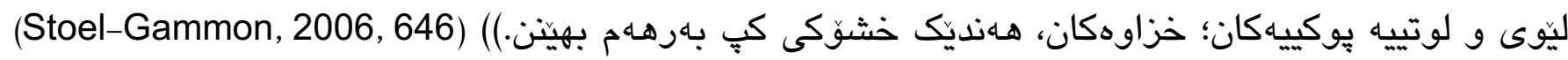

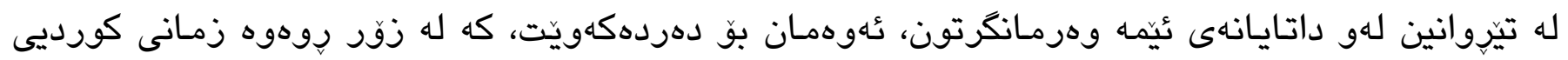

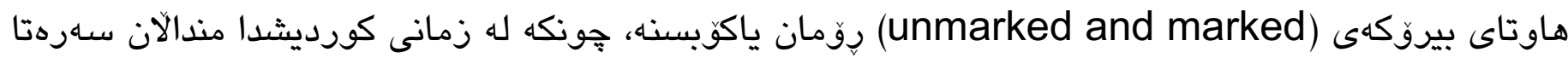

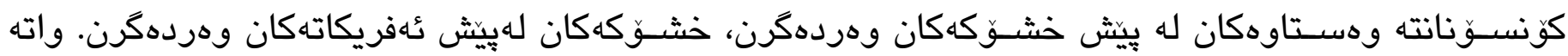

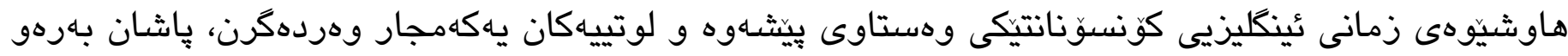

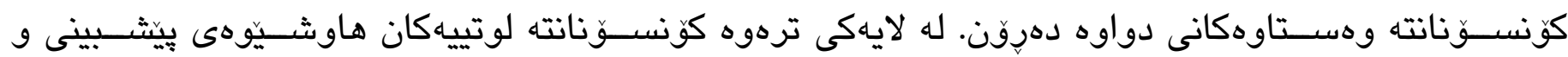

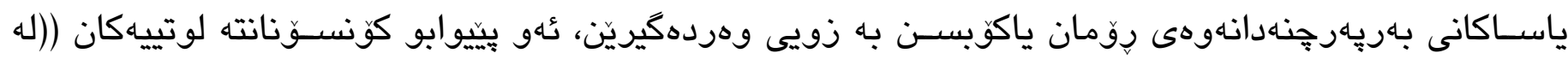

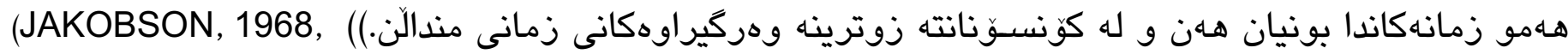

يهكيك لهو بابهتانهى، كه له (unmarked and marked) جهختى لهسهر دهكريتهوه، بابهتى زوّر بهكارهينانى

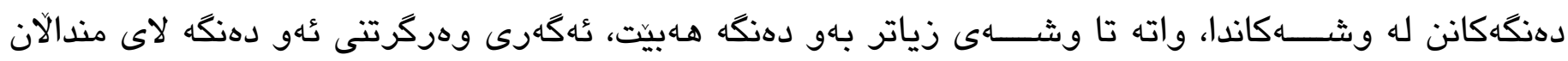

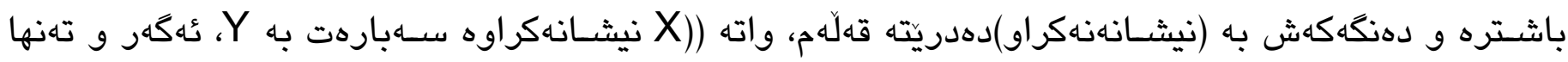

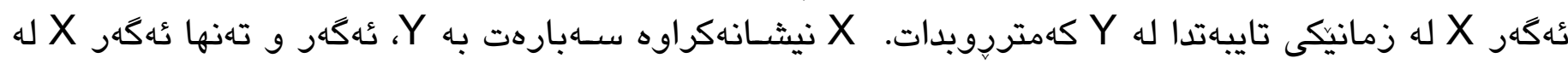

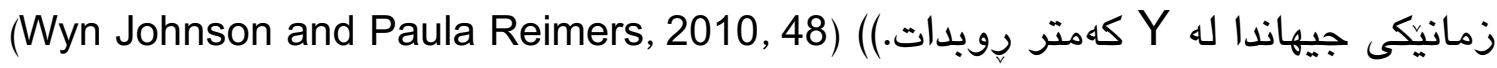

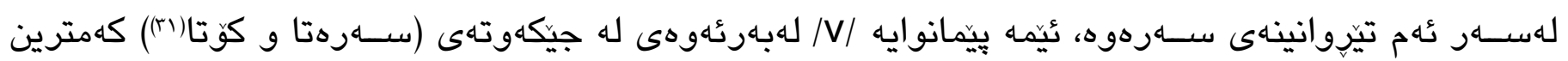

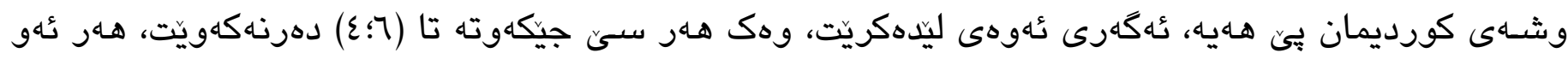

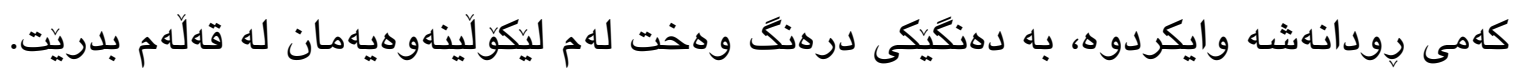

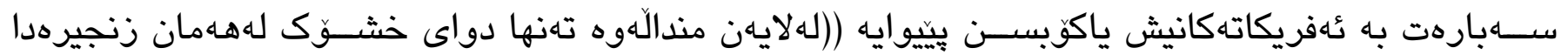

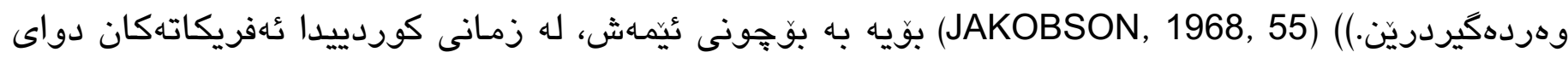

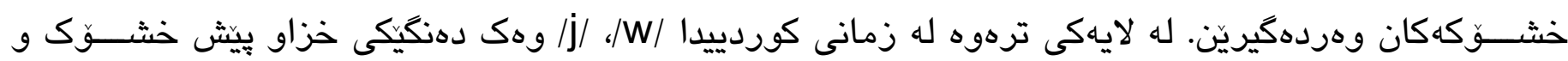

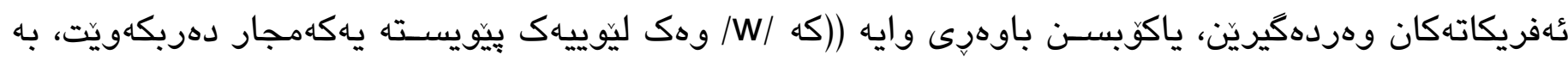

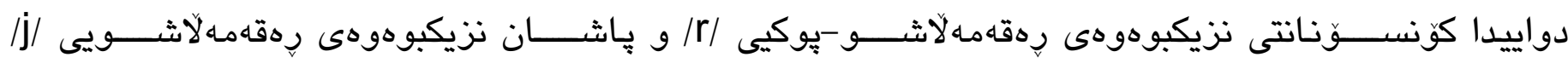

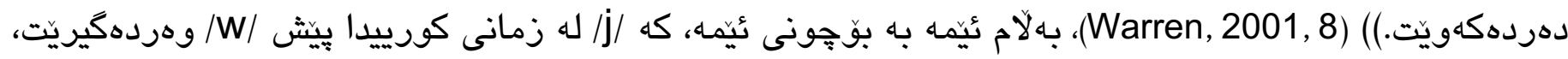

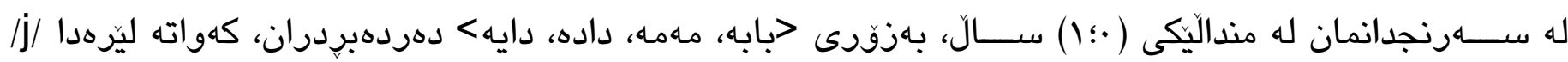

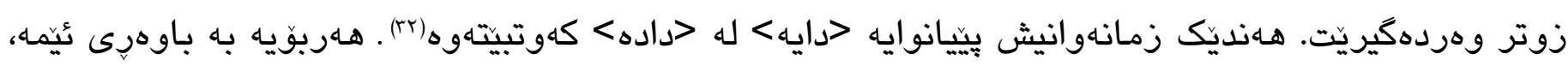

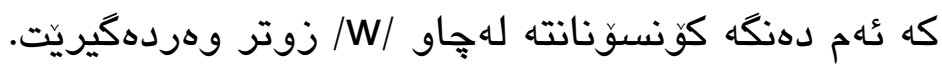

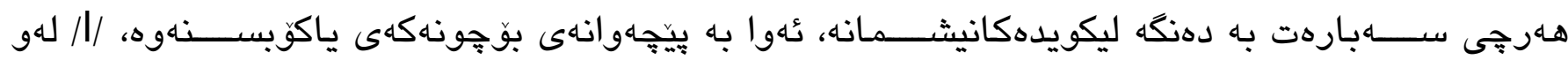

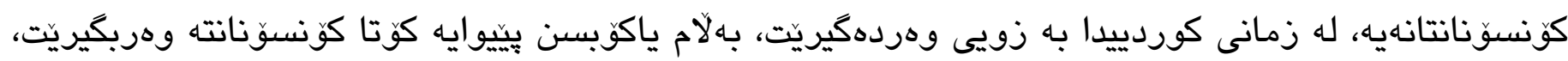

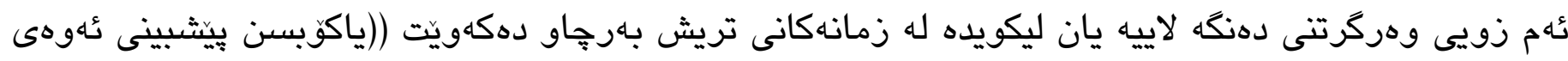

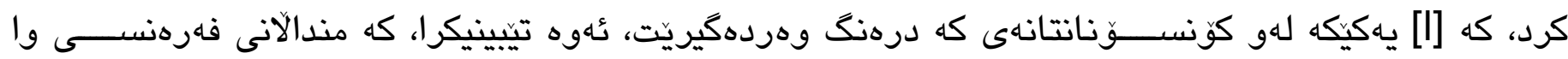

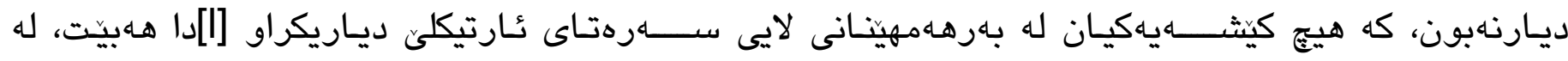

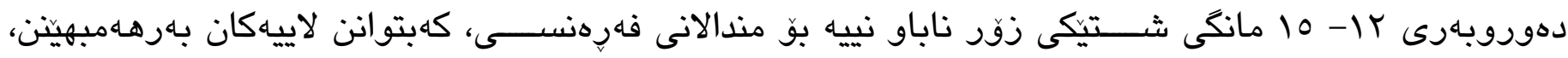

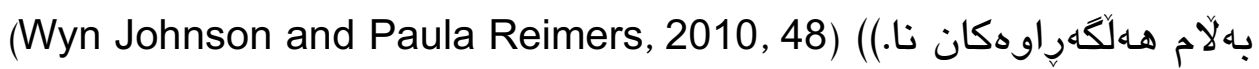




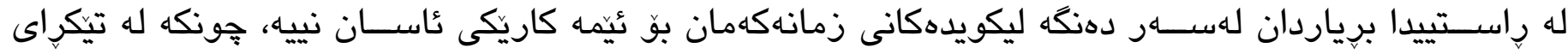

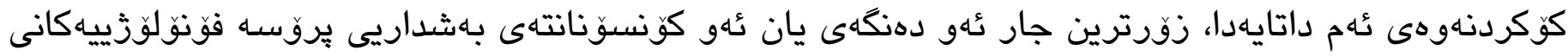

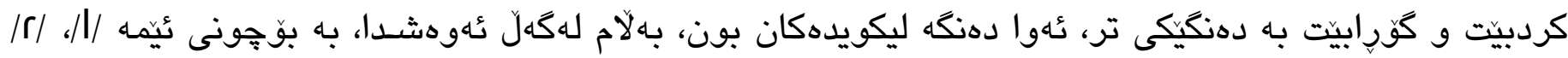

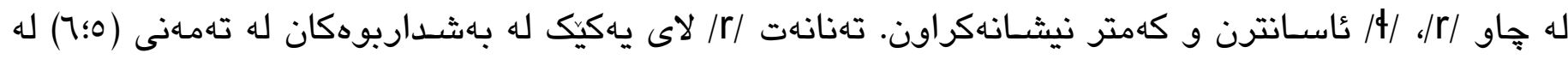

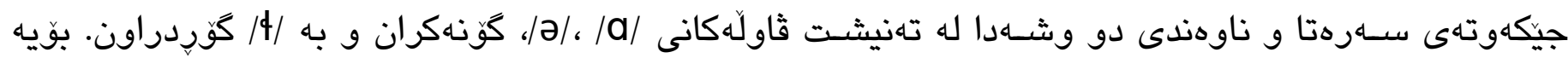

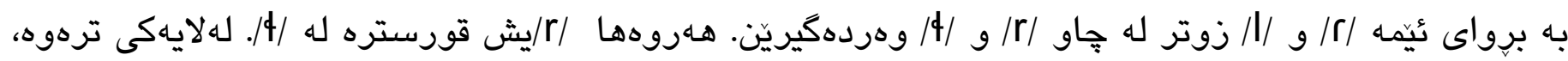

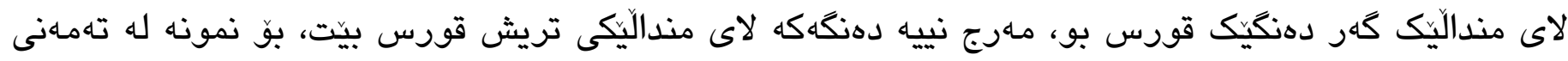

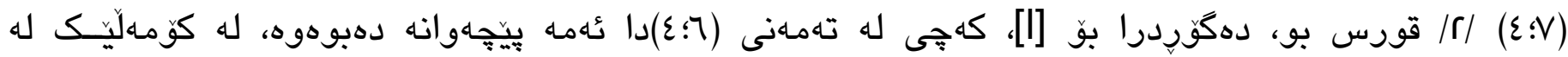
كوتنه كاندا /// دهبو به [r]

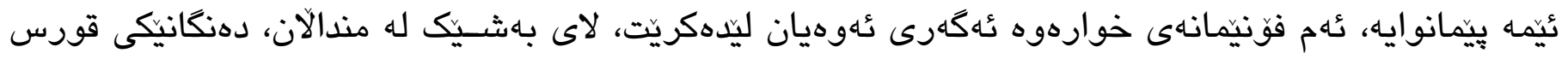

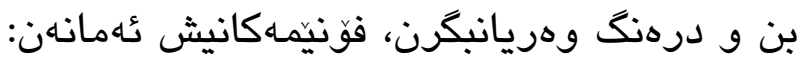

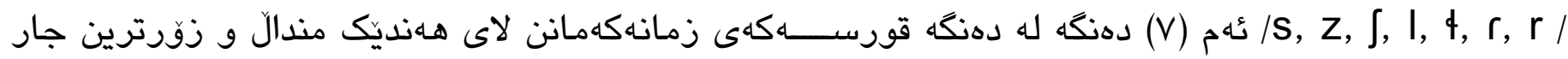

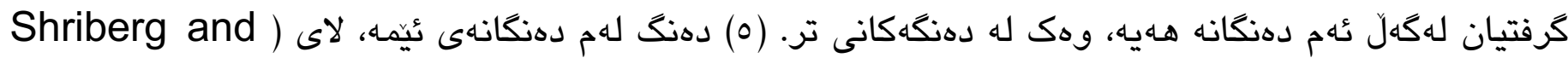
(Kwiatkowski

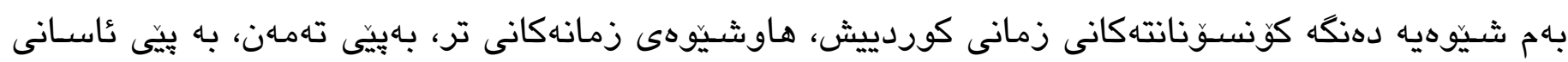

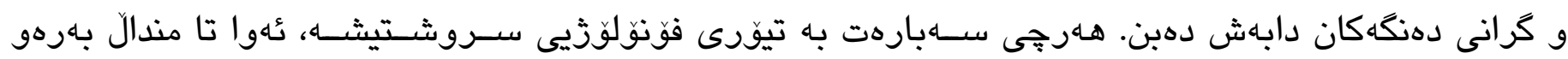

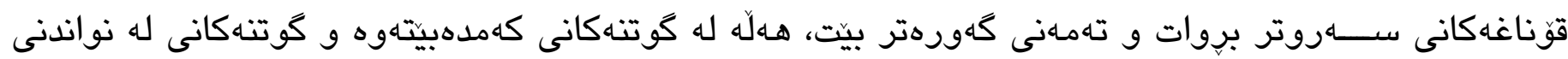

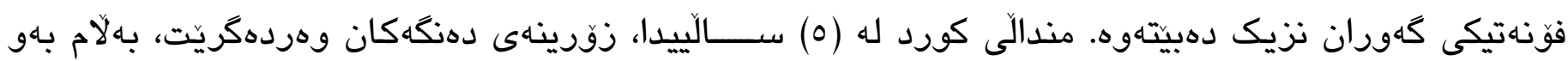

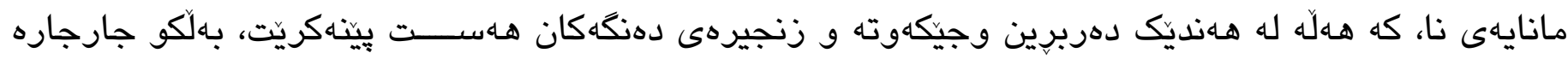

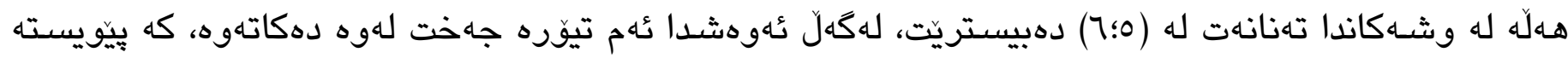

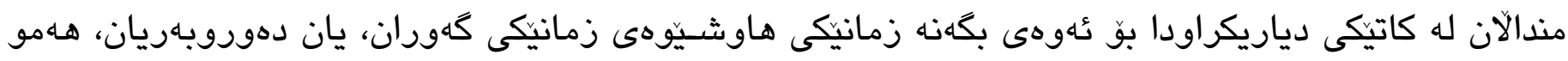

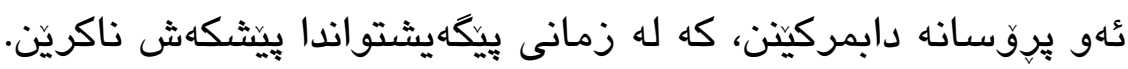

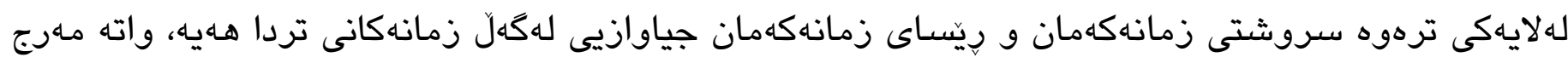

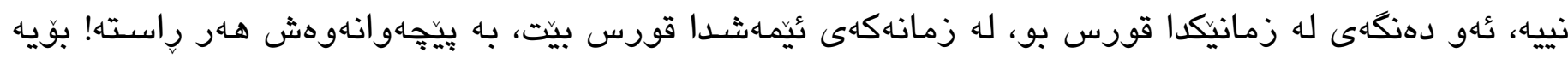

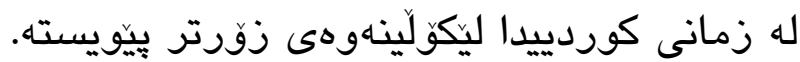

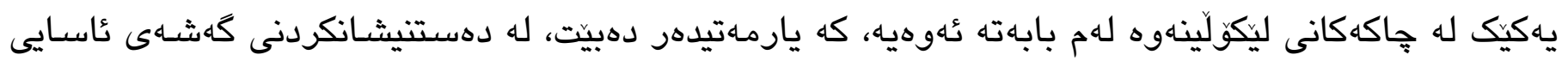

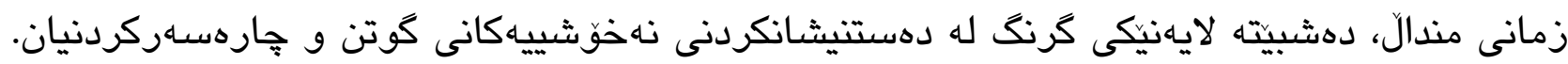




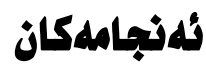

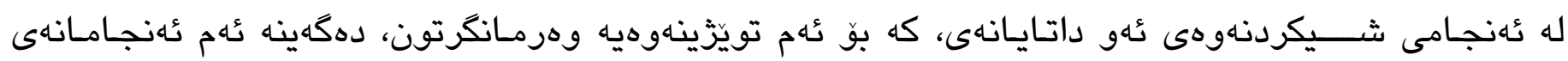
خوارهوه:

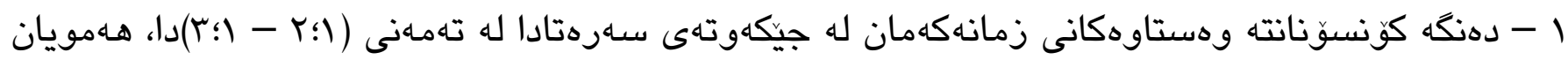
وهردهيريّن.

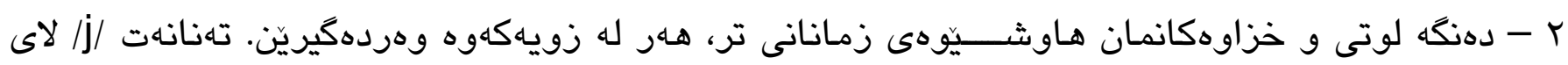

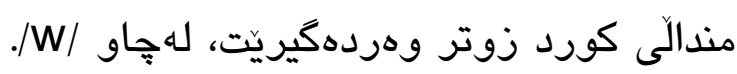

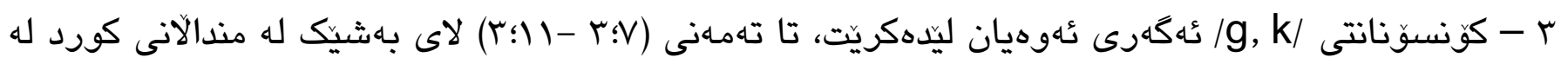

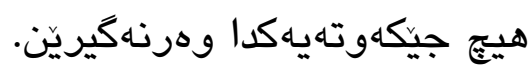

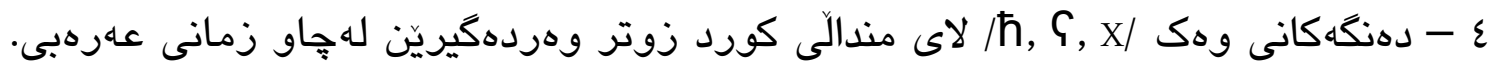

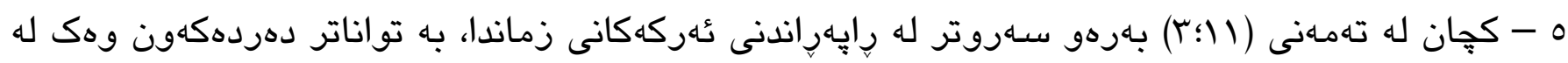
كوران.

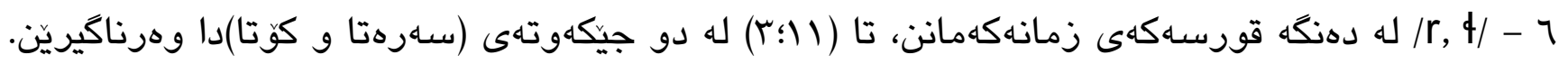

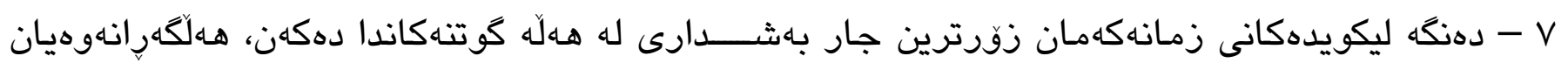

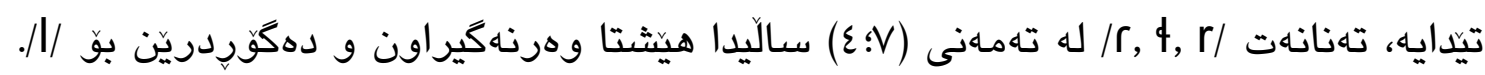

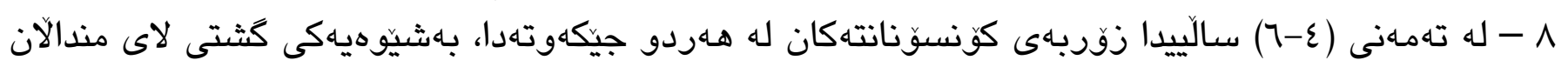
وهردهكيريَن. به

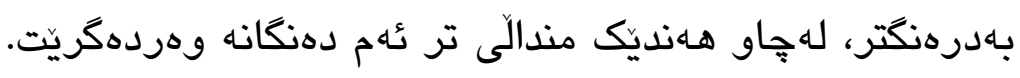




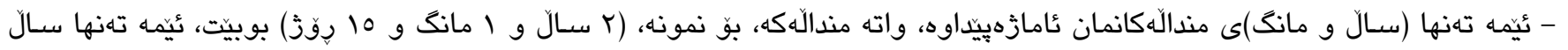

و مانكهكهمان ئامازه ييداوه.

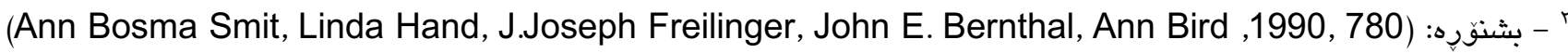

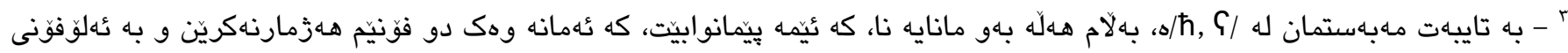

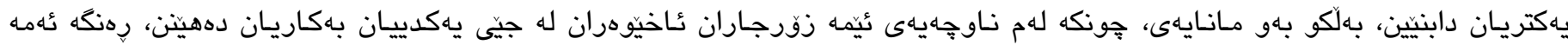

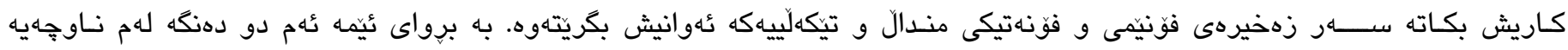

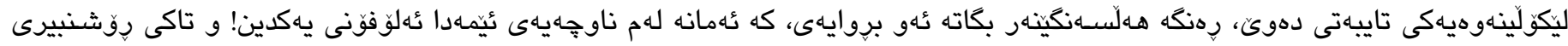

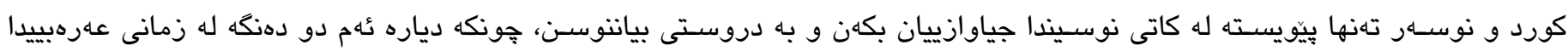
مانا دهكوّن!

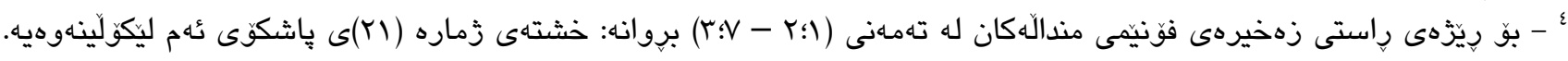

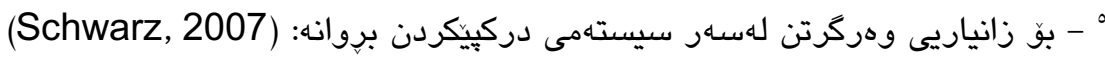

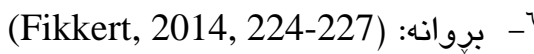

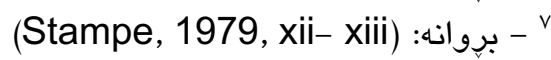

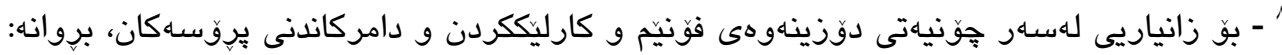

(Patricia, 1995, 62- 67 ) (Donegan, 1985) (Donegan, 2015, 44-46)

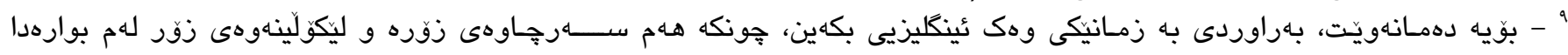

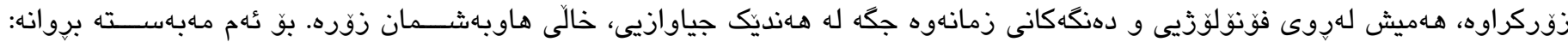

(Robinson Paulmony, Shivan Mawlood Hussein, 2019)

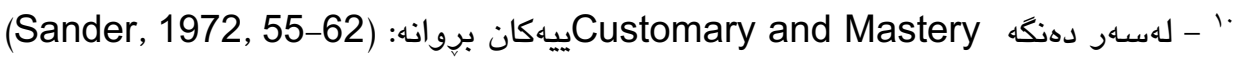

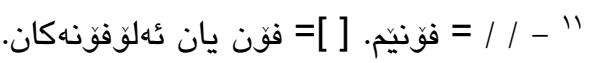

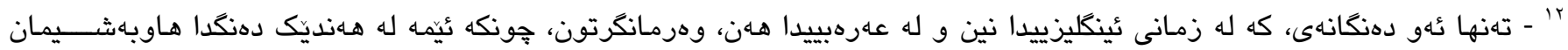

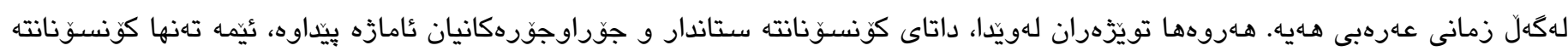

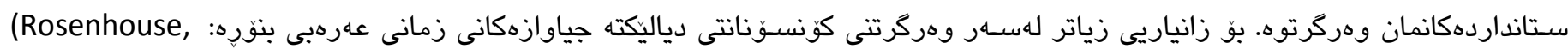
2000, 126-130), (Al-Awaji, 2014, 33)

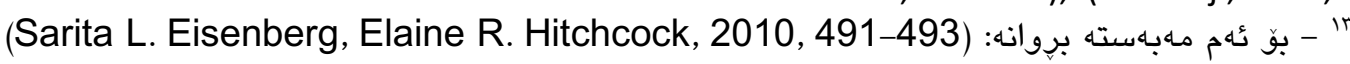

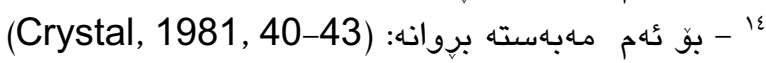
10

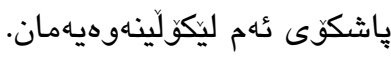

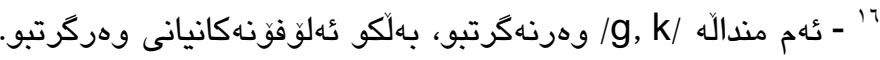

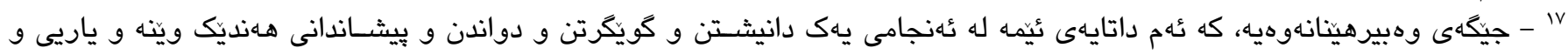

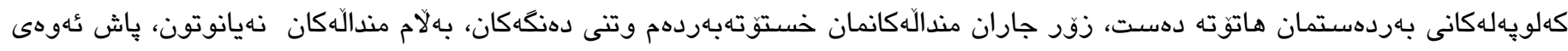

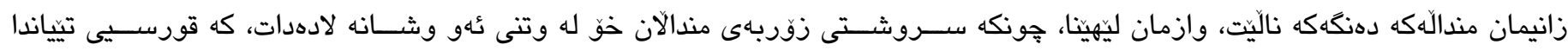

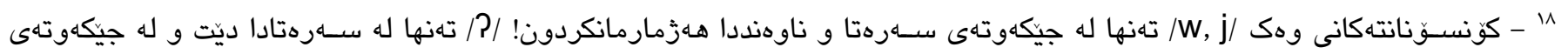

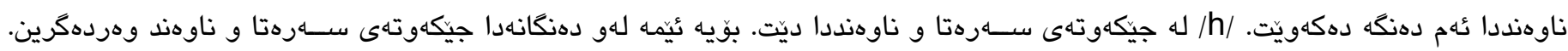

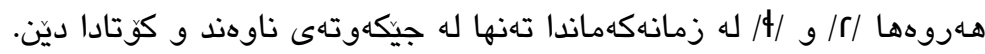

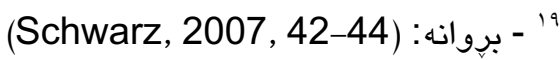

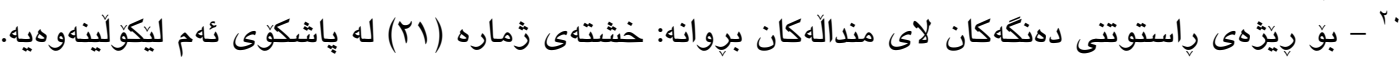

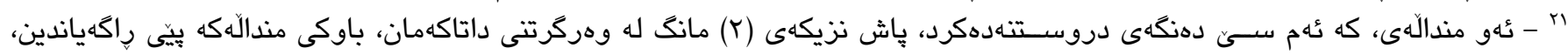

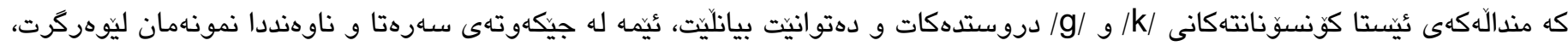

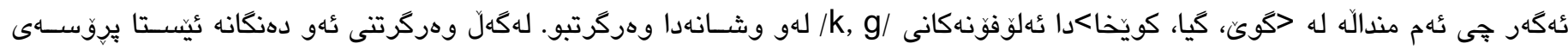

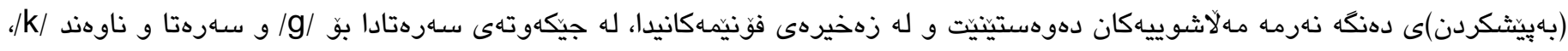




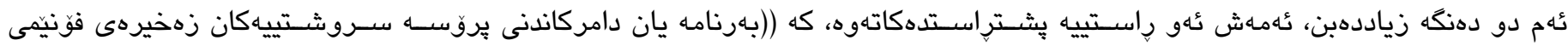

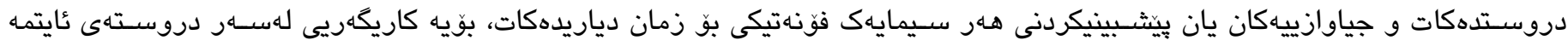

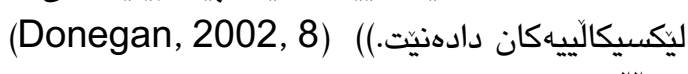

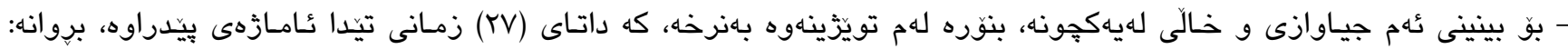

(Sharynne Mcleod and Kathryn Crowe, 2018)

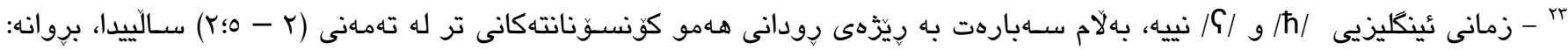

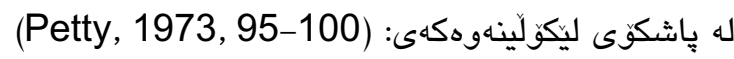

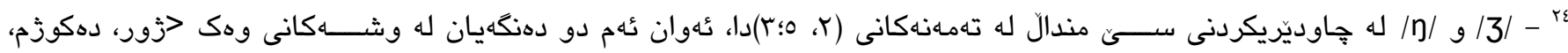

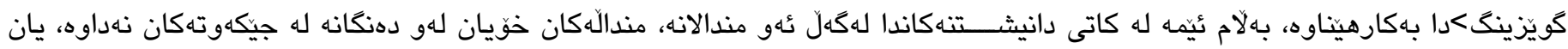

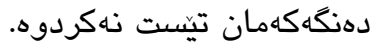

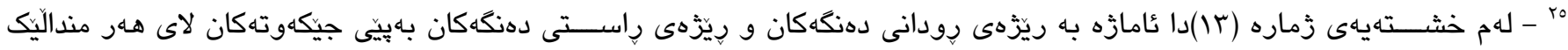

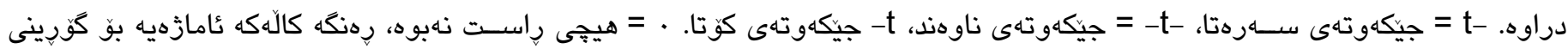

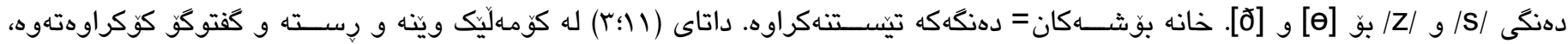

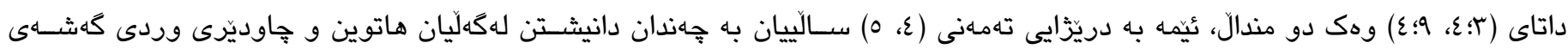

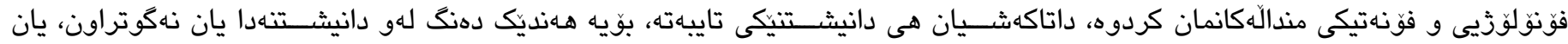

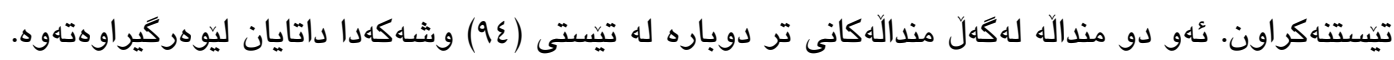

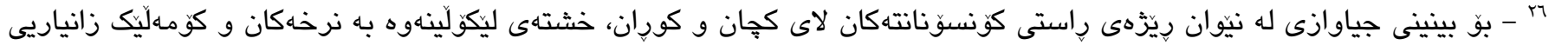

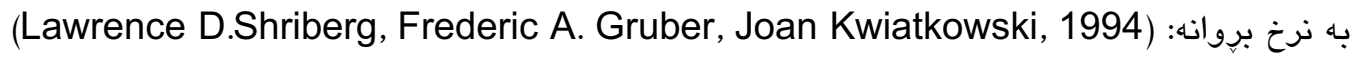

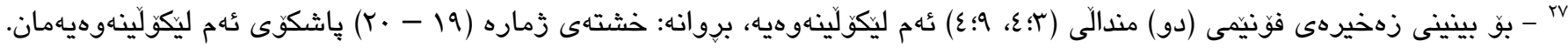

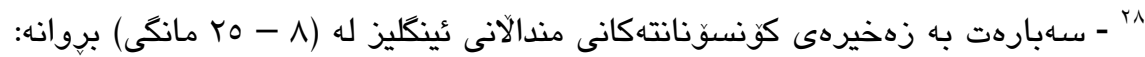
(John E. Bernthal, Nicholas W. Bankson, Peter Flipsen Jr, 2017, 71)

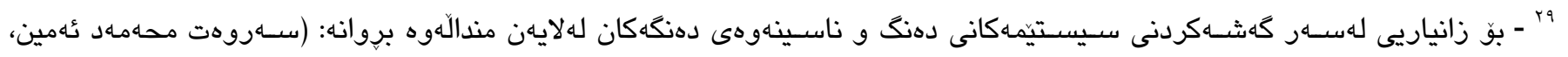

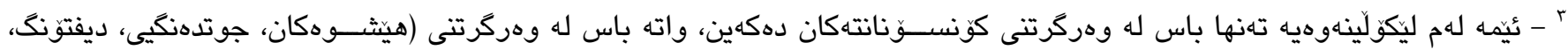

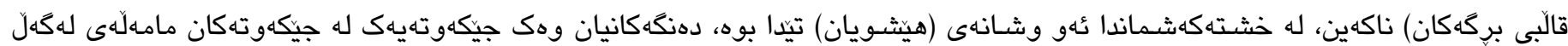

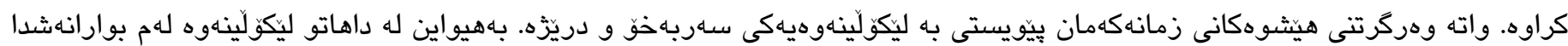

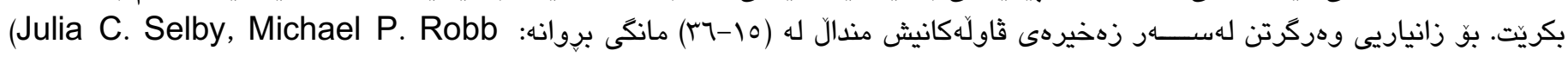
and Harvey R. Gilbert, 2000)

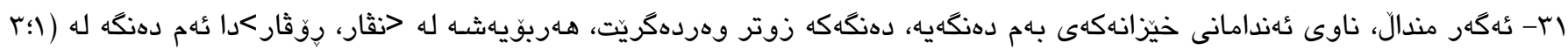

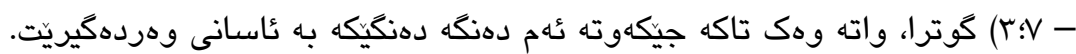

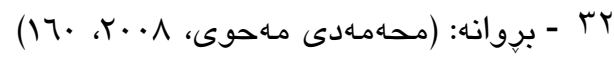

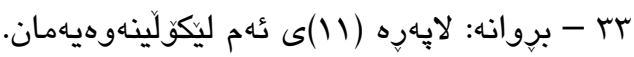




\title{
Acquiring Consonant Phonemes by Kurdish Children
}

\author{
Karzan Khidir Ismael \\ Department of Kurdish, College of Education, University of Raparin, Rania, Kurdistan \\ Region, Iraq. \\ E-mail: karzankhdir2@gmail.com
}

\section{Sherwan Husen Hamad}

Department of Kurdish, College of Basic Education, University of Raparin, Rania, Kurdistan Region, Iraq

E-mail: Sherwan983@uor.edu.krd

\begin{abstract}
:
Natural phonology and the theory of Roman Jacobson are crucial in acquiring language phonemes. In this paper which is entitled "acquiring consonant phonemes by Kurds children", we will endeavor based on the above theories, to focus on acquiring consonant phonemes in Kurdish language from age two years and a month to the age of six years and 5 months. We will also review the studies and methods that deal with acquiring language phonemes.

The importance of this study is to find out at what age do the Kurds children obtains all consonant phonemes, or what are the difficult phonemes for Kurds children which acquire them at a later time. Is there a gender difference in acquiring phonemes among children? The present paper beside these objectives also aims at catching the interests of those who investigate Kurdish language, in order to conduct more research's on this matter especially children phonology to determine the natural development of children language.
\end{abstract}

Keywords: Natural Phonology, Marked and Unmarked Sounds, Acquiring Consonant Phonemes. 


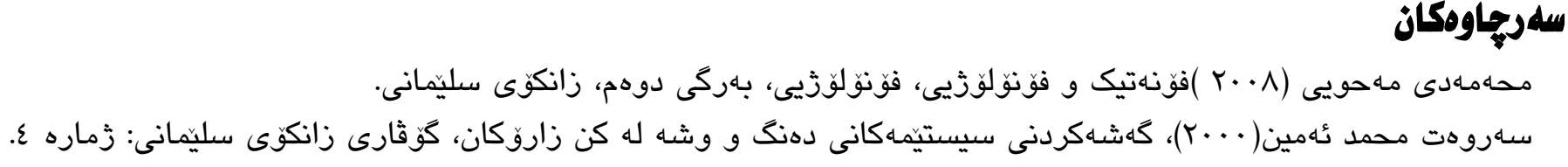

Adele W.Miccio, Dennis R. Ingrisano (2000) "The Acquisition of Fricatives and Affricates Evidence From a Disordered Phonological System." American Journal of Speech- Language Pathology 9, 214-229.

Aitchison, Jean (1987) Words in the Mind, An Introduction to the Mental Lexicon. First. Basil Blackwell.

Al-Awaji, Nisreen Naser (2014) Speech Production in Arabic Speaking Children with Operated Cleft Palate, Doctor of Philosophy. The University of Sheffield.

Ann Bosma Smit, Linda Hand, J.Joseph Freilinger, John E. Bernthal, Ann Bird (1990) "THE IOWA ARTICULATION NORMS PROJECT AND ITS NEBRASKA REPLICATION." Journal Of Speech and Hearing Disorder 55, 779-798.

BARBARA DODD, ALISON HOLM, ZHU HUA and SHARON CROSBIE (2003) "Phonological development: a normative study of British English- Speaking children." Clinical Linguistics and Phonetics (Taylor and francis healthsciences) 17, No.8, 617-643.

Bowen, Caroline (2015) Children's Speech Sound Disorders, Second, WILY Blackwell.

Crystal, David (1981) Disorders of Human Communication 3. Edited by F. Winckel,, B.D Wyke G.E.Arnold. Springer- Verlag Wien GmbH.

Diaz-Campos, Manuel Antonio (2001) ACUISTION OF PHONOLOGICAL STRUTURE AND SOCIOLINGUISTIC VARABLES: A quantitative analysis of Spanish Consonant Weakening In Venezuelan Children's Speech, Dissertation . The Ohio State Unuiversity .

Donegan, Patricia Jane (1985) How Learnable is Phonology .

Donegan, Patricia (2002) "Phonological Processes and Phonetic Rules."

Donegan, Patricia (2015) "The Emergence of Phonological Representation." In The Handbook of Language Emergence, by Brian MacWhinney and William O'grady. WILEY Blackwell.

Elizabeth M.Prather, Dona Lee Hedrick, Carolyn A. Kern (1990-1991). "Articulation Development in Children Aged Two to Four Years." National Student Speech Language Hearing Association Journal $18,96-102$.

Fikkert, Paula (2014) "Acquisition of Phonology." In The First Glot International State-of-the-Article Bood, 221-250. De Gruter Mouton.

FOX, ANNETTE V ( 2000) THE ACQUISITION OF PHONOLOGY AND THE CLASSIFICATION OF SPEECH DISORDERS IN GERMAN- SPEAKING CHILDREN, Doctor . Newcastle University Library .

Ingram, David (1989) First Language Acquision, Method, Description and Explanation. First. Cambridge University Press. 
JAKOBSON, ROMAN9 (1968) CHILD LANGUAGE APHASIA AND PHONOLOGICAL UNIVERSALS. First. MOUTON PUBLISHERS.

John E, Bernthal, Nicholas W, Bankson, Peter Flipsen Jr (2016) Articulation And Phonological Disorders, Speech Sound Disorders in Children,Eighth, Pearson.

Julia C. Selby, Michael P. Robb and Harvey R. Gilbert (2000) "Normal Vowel articulation between 15 and 36 months of age." Clinical Linguistics and Phonetics 14, 255-265.

Kathryn Crowe, Sharynne Mcleod (2020) "Childre's English Consonant Acquisition in the United States: A Review." American Journal Of Speech- Language Pathology 29, 2155-2169.

Lawrence D. Shriberg, Joan Kwiatkowski (1994) "Developmental Phonological Disorders I: A Clinical Profile." Journal of Speech and Hearing Research 37, 1100-1126.

Lawrence D.Shriberg, Frederic A. Gruber, Joan Kwiatkowski (1994) "Developmental Phonological Disorders III: Long-Term Speech-Sound Normalization." Journal of Speech and Hearing Research 37, 11511177.

Mousa M. Amayreh, Alice T. Dyson (1998) "The Acquistion of Arabic Consonants." Joournal of Speech, Language, and Hearing Research 41, 642-653.

Patricia, Donegan (1995) "The innateness of phonemic perception." Edited by Vida Samiian. WECOL 7 (Proceedings of the 24th Western Conference on Linguistics. Fresno, CA: Western Conference on Linguistics, 59-69.

Petty, Nancy Augstat (1973) The Articulation Proficiency of Two-Year-Old Children.

Robinson Paulmony, Shivan Mawlood Hussein (2019) "Phonological and Grammatical Similarities betwwen English and Kurdish Language: Why English Learning is Easier for Kurdish." Universal Journal of Educational Research 7, 2705-2709.

Rosenhouse, Judith (2000) "THE ACQUISITION OF ARABIC AS MOTHER TONGUE (MAINLY IN ISRAEL)." Oriente Moderno 119-151.

Sampson, Geoffrey (1980) Schools of Linguistics. First. Stanford University Press.

Sander, Eric K (1972) "When Are Speech Sounds Learned?" Journal of Speech and hearing disorders ASHA.

Sarita L. Eisenberg, Elaine R. Hitchcock (2010) "Using Standardized Tests to Inventory Consonant and Vowel Production: A Comparison of 11 Tests of Articulation and Phonology." Language, Speech, and Hearing in School (American Speech-Language- Hearing Association) 41, 488-503.

Schwarz, Iris-Corinna (2007) Speech Perception,Phonolgical Sensitivity, and Articulation in Early Vocabulary Development, Doctor of Philosophy . University of Western Sydney.

Shahla Fatemi Syadar, Talieh Zarifian, Yahya Modarresi, Muhamad Sediq Zahedi, Mona Ebrahimipur, Akbar Biglarian (2018) "Percentage of Consonants Correct for 3-5 Years Old Kurdish- Speaking Children With Middle Kurmanji- Mukryani Dialect." Iranian Rehabilitation Journal 16.

Sharynne Mcleod and Kathryn Crowe (2018) "Children's Consonant Acquisition in 27 Languages: A CrossLinguistic Review." American Journal of Speěeh-language Pathology 27, 1546-1571. 
Smit, Ann B (1986) "Ages of Speech Sound Acquisition: Comparisons and Critiques of Several Normative Studies." Language, Speech, and Hearing Services in Schools 17, 175-186.

Stampe, David (1979) A dissertation on Natural Phonology. Garland Publishing.

Stoel-Gammon, C (2006) "Infancy: Phonological Development." (University of Washington).

Storkel, Holly L (2018) "The Compexity Approach to Phonological Treatment: How to Select Treatment Targets." Language, Speech, and Hearing Service in schools 1-19.

Templin, Mildred C (1947) "Spontaneous Versus Imitated Verbalization In Testing Articulation In Prescool Children." JOURNAL OF SPEECH DISORDES 12: 293-300.

Warren, Sandra (2001) Phonological Acquisition and Ambient Language A Corpus Based Cross-Linguistic Exploration, Doctor of Philosophy. Vols. 2, Appendices.

Weybright, Glenn Douglas (1974) An investigation of the development of the phonemes /t/ and / $/$ / in the speech of preschool children, Master of Science Speech. Portland State University.

William O'Grady and Sook Whan Cho (2005) First Language Acquisition.

Wyn Johnson and Paula Reimers (2010) Pattern in child phonology. Edinburg University Press.

\begin{tabular}{|c|c|c|c|c|}
\hline كوَتا Final & ناوهراست Medial & سلهرهتا Initail سل & 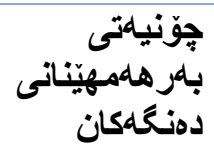 & r؟؛ كور \\
\hline$b, p, t, k, g, q$ & $b, p, t, d, k, g, q$ & $b, p, t, d, k, g, q, ?$ & 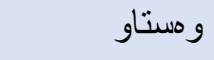 & \\
\hline \multirow[t]{2}{*}{$m, n, \eta$} & $\mathrm{m}, \mathrm{n}$ & $\mathrm{m}, \mathrm{n}$ & لوتيى & \\
\hline & $j, w$ & $\mathrm{j}, \mathrm{w}$ & 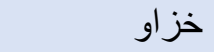 & \\
\hline $\int, x, \hbar$ & $\mathrm{f}, \mathrm{v}, \int, 3, \mathrm{x}, \mathrm{\gamma}, \hbar \mathrm{\hookrightarrow}, \mathrm{h}$ & $f, \int, 3, x, \hbar, h$ & خشُوك & \\
\hline$t 5, d s$ & $t 5, d 3$ & $t 5, d 3$ & ئافريكات & \\
\hline 1,4 & 1,4 & I & لايى & \\
\hline$r, r$ & $r, r$ & $r$ & للهروك & \\
\hline
\end{tabular}

خشتهى زماره (9 (): زهخيرهى فُونيمى له تهمانى (T؟ع) 


\begin{tabular}{|c|c|c|c|}
\hline كوّتا Final & ناوهراست Medial & سلرهتا Initial & 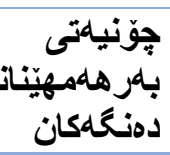 \\
\hline$b, p, t, d, k, g, q$ & $b, p, t, d, k, g, q$ & $\begin{array}{l}b, p, t, d, k, g \\
q, ?\end{array}$ & و وستاو \\
\hline \multirow[t]{2}{*}{$m, n, \eta$} & $\mathrm{m}, \mathrm{n}$ & $\mathrm{m}, \mathrm{n}$ & لوتيى \\
\hline & $w, j$ & $w, j$ & خزاو \\
\hline$s, \int, z, 3, x, \hbar$ & $\begin{array}{l}f, s, \int, z, 3, x, \varsigma, \\
\hbar, h\end{array}$ & $\begin{array}{l}f, s, \int, z, 3, x \\
\varsigma, \hbar, h\end{array}$ & خشّوك \\
\hline$t 5, d 3$ & $d 3$ & $d 3, t$ & ئدفريكات \\
\hline 1,4 & 1,4 & 1 & لايى \\
\hline$r, r$ & $r, r$ & $r$ & 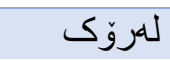 \\
\hline
\end{tabular}

خشتهى زماره (·r): زهخيرهى فوّنيمى له (9؛ ع)

\begin{tabular}{|c|c|c|c|c|c|c|c|c|c|c|}
\hline \multicolumn{2}{|c|}{$\Gamma ! V$} & \multicolumn{2}{|c|}{$r ! 1$} & \multicolumn{2}{|c|}{$r \leqq \Lambda$} & \multicolumn{2}{|c|}{ 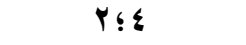 } & \multicolumn{2}{|c|}{$r \leqq 1$} & \multirow[b]{2}{*}{ دهنگ } \\
\hline$\%$ & زماره & $\%$ & زماره & $\%$ & زماره & $\%$ & زماره & $\%$ & زماره & \\
\hline \multirow[t]{3}{*}{$\Lambda$. } & 0 & $1 \ldots$ & 0 & $1 \ldots$ & V & $\Lambda \Lambda$ & 9 & $1 \ldots$ & 0 & b- \\
\hline & & $1 \ldots$ & V & $1 \ldots$ & r & $1 \ldots$ & $r$ & $1 \ldots$ & r & $-b-$ \\
\hline & & $1 \ldots$ & $r$ & $1 \ldots$ & 1 & & & & & $-b$ \\
\hline $1 \ldots$ & 0 & $1 \ldots$ & $r$ & $1 \ldots$ & r & vo & $\varepsilon$ & 0. & r & $p-$ \\
\hline $1 \ldots$ & r & $1 \ldots$ & r & $1 \ldots$ & 1 & $1 \ldots$ & r & $1 \ldots$ & 1 & $-p-$ \\
\hline $1 \ldots$ & 1 & $1 \ldots$ & $r$ & $1 \ldots$ & $r$ & . & 1 & & & $-p$ \\
\hline $1 \ldots$ & 1 & 77 & $r$ & $1 \ldots$ & $r$ & & & $1 \ldots$ & $r$ & $t-$ \\
\hline 79 & $r$ & $1 \ldots$ & 1 & $1 \ldots$ & $r$ & OV & V & $1 \ldots$ & $r$ & -t- \\
\hline $1 \ldots$ & $r$ & $1 \ldots$ & $r$ & & & $1 \ldots$ & $r$ & $1 \ldots$ & 1 & $-t$ \\
\hline $1 \ldots$ & 0 & $1 \ldots$ & 9 & $1 \ldots$ & $r$ & $1 \ldots$ & 0 & $1 \ldots$ & $r$ & d- \\
\hline \multirow[t]{2}{*}{$1 \ldots$} & $r$ & $1 \ldots$ & 0 & $1 \ldots$ & 1 & $1 \ldots$ & $\varepsilon$ & $1 \ldots$ & $r$ & $-d-$ \\
\hline & & $1 \ldots$ & 1 & & & & & & & $-d$ \\
\hline$r$. & 0 & $1 \ldots$ & 7 & $1 \ldots$ & $r$ & vo & $\Lambda$ & 0. & $r$ & k- \\
\hline . & $r$ & $1 \ldots$ & 0 & $1 \ldots$ & $r$ & $1 \ldots$ & 0 & vo & $\varepsilon$ & $-k-$ \\
\hline • & $r$ & $1 \ldots$ & $r$ & $1 \ldots$ & 1 & 0. & $r$ & & & $-\mathrm{k}$ \\
\hline 0 . & $\varepsilon$ & $1 \ldots$ & $r$ & $1 \ldots$ & $r$ & $1 \ldots$ & $\varepsilon$ & 0. & $r$ & g- \\
\hline \multirow[t]{2}{*}{. } & $r$ & 0. & $r$ & $1 \ldots$ & 1 & $1 \ldots$ & $r$ & & & $-g-$ \\
\hline & & & & & & & & & & $-g$ \\
\hline . & $\varepsilon$ & $1 \ldots$ & 1 & & & 77 & $r$ & & & $\mathrm{q}^{-}$ \\
\hline \multirow[t]{2}{*}{. } & 1 & $1 \ldots$ & 1 & & & $1 \ldots$ & $r$ & · & 1 & $-q-$ \\
\hline & & & & & & $\cdot$ & 1 & $1 \ldots$ & 1 & $-q$ \\
\hline $1 \ldots$ & $r$ & $1 \ldots$ & $\varepsilon$ & $1 \ldots$ & 0 & $1 \ldots$ & $\varepsilon$ & $1 \ldots$ & $r$ & ?- \\
\hline $1 \ldots$ & $\varepsilon$ & $1 \ldots$ & $\Lambda$ & $1 \ldots$ & $r$ & $1 \ldots$ & V & $1 \ldots$ & 1 & m- \\
\hline $1 \ldots$ & V & $1 \ldots$ & 0 & $1 \ldots$ & $r$ & 77 & $r$ & $1 \ldots$ & $r$ & $-m-$ \\
\hline $1 \ldots$ & $\Lambda$ & $1 \ldots$ & $\varepsilon$ & $1 \ldots$ & $r$ & $1 \ldots$ & $r$ & $1 \ldots$ & $r$ & $-\mathrm{m}$ \\
\hline$\Lambda \mu$ & 7 & $1 \ldots$ & $r$ & & & $1 \ldots$ & $r$ & & & $\mathrm{n}-$ \\
\hline $1 \ldots$ & 0 & $1 \ldots$ & $\wedge$ & $1 \ldots$ & 0 & $\Lambda$. & 0 & $1 \ldots$ & 0 & $-n-$ \\
\hline \multirow[t]{3}{*}{$1 \ldots$} & 7 & $1 \ldots$ & $r$ & $1 \ldots$ & 1 & $1 \ldots$ & $r$ & & & $-n$ \\
\hline & & & & & & . & 1 & & & $-\eta$ \\
\hline & & & & & & & & $1 \ldots$ & 1 & W- \\
\hline \multirow[t]{2}{*}{ vo } & $\varepsilon$ & $1 \ldots$ & V & $1 \ldots$ & V & A. & 0 & $1 \ldots$ & 0 & $-W-$ \\
\hline & & $1 \ldots$ & 1 & $1 \ldots$ & 1 & & & & & j- \\
\hline \multirow[t]{2}{*}{$1 \ldots$} & $r$ & $1 \ldots$ & $r$ & $1 \ldots$ & $r$ & 10 & V & $1 \ldots$ & 7 & $-j-$ \\
\hline & & $1 \ldots$ & r & $1 \ldots$ & 1 & & & & & $f-$ \\
\hline
\end{tabular}




\begin{tabular}{|c|c|c|c|c|c|c|c|c|c|c|}
\hline \multirow[t]{3}{*}{$1 \ldots$} & 1 & $1 \ldots$ & 1 & $1 \ldots$ & 1 & & & $1 \ldots$ & 1 & $-f-$ \\
\hline & & $1 \ldots$ & 1 & & & & & & & $-f$ \\
\hline & & & & & & & & & & V- \\
\hline \multirow{2}{*}{$1 \ldots$} & 1 & $1 \ldots$ & 1 & $1 \ldots$ & 1 & $1 \ldots$ & 1 & & & $-V-$ \\
\hline & & & & & & & & & & $-v$ \\
\hline $1 \ldots$ & 7 & ro & $\varepsilon$ & $1 \ldots$ & $r$ & . & $\varepsilon$ & $1 \ldots$ & $r$ & S- \\
\hline $1 \ldots$ & $r$ & $\cdot$ & 0 & 77 & $r$ & . & $r$ & 0. & r & -s- \\
\hline $1 \ldots$ & 1 & & & & & . & 1 & & & $-S$ \\
\hline $1 \ldots$ & 1 & $1 \ldots$ & 1 & & & . & 1 & & & Z- \\
\hline $1 \ldots$ & $r$ & 0. & $\varepsilon$ & $1 \ldots$ & $r$ & . & 1 & · & 1 & $-z-$ \\
\hline \multirow[t]{2}{*}{$1 \ldots$} & $r$ & . & $r$ & & & $\cdot$ & 1 & & & $-z$ \\
\hline & & & & $1 \ldots$ & r & $1 \ldots$ & 1 & $1 \ldots$ & $r$ & $\int-$ \\
\hline $1 \ldots$ & 1 & . & $r$ & 0. & $r$ & $1 \ldots$ & $r$ & & & $-\int-$ \\
\hline \multirow[t]{4}{*}{$1 \ldots$} & $r$ & . & 1 & $1 \ldots$ & 1 & $\cdot$ & 1 & $1 \ldots$ & 1 & $-\int$ \\
\hline & & & & & & & & & & $3-$ \\
\hline & & & & & & & & & & $-3-$ \\
\hline & & & & & & & & & & -3 \\
\hline $1 \ldots$ & 1 & & & $1 \ldots$ & T & 0. & $r$ & & & $x-$ \\
\hline$\cdot$ & 1 & $1 \ldots$ & 1 & $1 \ldots$ & 1 & & & $1 \ldots$ & 1 & $-x-$ \\
\hline \multirow[t]{4}{*}{$1 \ldots$} & 1 & & & & & $1 \ldots$ & $r$ & & & $-x$ \\
\hline & & & & & & & & & & $\gamma-$ \\
\hline & & & & & & & & & & $-\gamma-$ \\
\hline & & & & & & & & & & $-\gamma$ \\
\hline \multirow[t]{3}{*}{$1 \ldots$} & 1 & $1 \ldots$ & $r$ & & & & & & & $\hbar-$ \\
\hline & & $1 \ldots$ & 1 & . & 1 & & & & & $-\hbar-$ \\
\hline & & & & & & & & & & $-\hbar$ \\
\hline \multirow[t]{5}{*}{ · } & 1 & $1 \ldots$ & $r$ & & & & & $1 \ldots$ & 1 & S- \\
\hline & & $1 \ldots$ & 1 & & & & & & & -ৎ- \\
\hline & & & & & & & & & & $-\zeta$ \\
\hline & & $1 \ldots$ & $r$ & $1 \ldots$ & $r$ & $1 \ldots$ & $r$ & $1 \ldots$ & $r$ & h- \\
\hline & & & & & & & & Vo & $\varepsilon$ & $-h-$ \\
\hline $1 \ldots$ & 1 & & & & & $1 \ldots$ & 1 & & & $d 3^{-}$ \\
\hline \multirow[t]{2}{*}{$1 \ldots$} & 1 & & & & & $1 \ldots$ & 1 & $1 \ldots$ & 1 & $-d z$ \\
\hline & & & & & & & & & & $-d 3$ \\
\hline $1 \ldots$ & $r$ & 77 & $r$ & $1 \ldots$ & 1 & . & $r$ & . & 1 & t- \\
\hline $1 \ldots$ & $r$ & & & & & . & 1 & . & $r$ & $-t 5$ \\
\hline $1 \ldots$ & 1 & $1 \ldots$ & 1 & & & . & 1 & & & $-t 5$ \\
\hline 0. & $r$ & • & $r$ & & & . & 1 & $1 \ldots$ & $r$ & I- \\
\hline$\Lambda$ & 0 & . & $\varepsilon$ & Vo & $\varepsilon$ & $1 \ldots$ & $\varepsilon$ & & & $-\mid-$ \\
\hline $1 \ldots$ & 1 & & & & & & & & & -1 \\
\hline 0. & $r$ & . & $\varepsilon$ & $1 \ldots$ & $r$ & $1 \ldots$ & $r$ & · & 0 & $-4-$ \\
\hline · & $r$ & & & & & . & 1 & . & 1 & -4 \\
\hline$\Lambda$. & 9 & $r$. & 1. & $1 \ldots$ & $\Lambda$ & . & $1 \varepsilon$ & 77 & $r$ & $-r-$ \\
\hline$\Lambda$. & 0 & ro & 0 & $1 \ldots$ & 1 & $1 \ldots$ & 1 & $1 \ldots$ & 1 & $-r$ \\
\hline$\cdot$ & 1 & $\cdot$ & $\varepsilon$ & & & $\cdot$ & 1 & $\cdot$ & $r$ & $r-$ \\
\hline \multirow[t]{2}{*}{. } & 1 & . & $\varepsilon$ & 77 & $r$ & . & $r$ & . & 1 & $-r-$ \\
\hline & & . & 1 & & & & & & & $-r$ \\
\hline
\end{tabular}

خشتهى زماره: ( (Y): رِيَّْهى راست وتنى دهنكهكان لاى مندالان. 